\title{
First Cosmology Results Using SNe Ia from the Dark Energy Survey: Analysis, Systematic Uncertainties, and Validation
}

D. Brout ${ }^{1}$ (D) D. Scolnic ${ }^{2}$, R. Kessler ${ }^{2,3}$ (1) , C. B. D’Andrea ${ }^{1}$ (1), T. M. Davis ${ }^{4}$, R. R. Gupta ${ }^{5}$, S. R. Hinton ${ }^{4}$ (i), A. G. Kim ${ }^{5}$, J. Lasker, ${ }^{2,3}$, C. Lidman $^{6}$ (1) E. Macaulay ${ }^{7}$, A. Möller ${ }^{6,8}$, R. C. Nichol ${ }^{7}$, M. Sako ${ }^{1}$, M. Smith ${ }^{9}$, M. Sullivan ${ }^{9}$, B. Zhang ${ }^{6,8}$, P. Andersen ${ }^{4,10}$, J. Asorey ${ }^{11}$, A. Avelino ${ }^{12}$, B. A. Bassett ${ }^{13,14}$, P. Brown ${ }^{15}$, J. Calcino ${ }^{4}$, D. Carollo ${ }^{16}$, P. Challis ${ }^{12}$, M. Childress ${ }^{9}$, A. Clocchiatti ${ }^{17}$, A. V. Filippenko ${ }^{18,19}$, R. J. Foley ${ }^{20}$, L. Galbany ${ }^{21}$, K. Glazebrook ${ }^{22}$ (1), J. K. Hoormann ${ }^{4}$, E. Kasai ${ }^{14,23}$, R. P. Kirshner ${ }^{24,25}$, K. Kuehn ${ }^{26}$, S. Kuhlmann ${ }^{27}$, G. F. Lewis ${ }^{28}$ (1) K. S. Mandel ${ }^{29}$, M. March ${ }^{1}$, V. Miranda ${ }^{30}$, E. Morganson ${ }^{31}$ (1), D. Muthukrishna ${ }^{6,8,32}$ (1) P. Nugent ${ }^{5}$ (10), A. Palmese ${ }^{33}$ (1), Y.-C. Pan ${ }^{34,35}$, R. Sharp ${ }^{6}$, N. E. Sommer, ${ }^{6,8}$ E. Swann ${ }^{7}$, R. C. Thomas ${ }^{5}$,

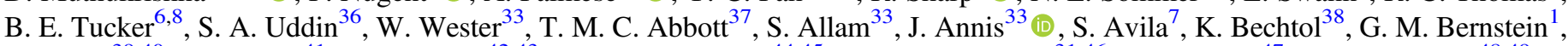
E. Bertin ${ }^{39,40}$, D. Brooks ${ }^{41}$, D. L. Burke ${ }^{42,43}$, A. Carnero Rosell ${ }^{44,45}$, M. Carrasco Kind ${ }^{31,46}$, J. Carretero ${ }^{47}$, F. J. Castander ${ }^{48,49}$ (1), C. E. Cunha ${ }^{42}$, L. N. da Costa ${ }^{45,50}$, C. Davis ${ }^{42}$, J. De Vicente ${ }^{44}$, D. L. DePoy ${ }^{15}$, S. Desai ${ }^{51}$, H. T. Diehl ${ }^{33}$, P. Doel ${ }^{41}$, A. Drlica-Wagner ${ }^{2,33}$ (D) T. F. Eifler ${ }^{30,52}$, J. Estrada ${ }^{33}$, E. Fernandez ${ }^{47}$, B. Flaugher $^{33}$, P. Fosalba ${ }^{48,49}$, J. Frieman ${ }^{2,33}$, J. García-Bellido ${ }^{53}$, D. Gruen ${ }^{42,43}$, R. A. Gruendl ${ }^{31,46}$ (1) G. Gutierrez ${ }^{33}$, W. G. Hartley ${ }^{41,54}$, D. L. Hollowood ${ }^{20}$ (1), K. Honscheid ${ }^{55,56}$, B. Hoyle ${ }^{57,58}$, D. J. James ${ }^{59}$, M. Jarvis ${ }^{1}$, T. Jeltema ${ }^{20}$, E. Krause ${ }^{30}$, O. Lahav ${ }^{41}$, T. S. Li ${ }^{2,33}$, M. Lima ${ }^{45,60}$, M. A. G. Maia ${ }^{45,50}$, J. Marriner ${ }^{33}$, J. L. Marshall ${ }^{15}$, P. Martini ${ }^{55,61}$ (1) F. Menanteau ${ }^{31,46}$, C. J. Miller ${ }^{62,63}$, R. Miquel ${ }^{47,64}$ (D), R. L. C. Ogando ${ }^{45,50}$, A. A. Plazas ${ }^{52}$, A. K. Romer ${ }^{65}$, A. Roodman ${ }^{42,43}$, E. S. Rykoff ${ }^{42,43}$, E. Sanchez ${ }^{44}$, B. Santiago ${ }^{45,66}$, V. Scarpine ${ }^{33}$, M. Schubnell ${ }^{63}$, S. Serrano ${ }^{48,49}$, I. Sevilla-Noarbe ${ }^{44}$, R. C. Smith ${ }^{37}$, M. Soares-Santos ${ }^{67}$ (1) F. Fobreira ${ }^{45,68}$,

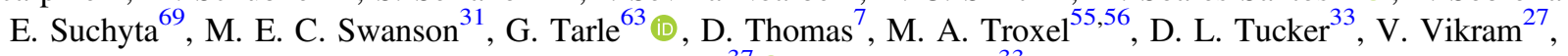
A. R. Walker ${ }^{37}$ (10, and Y. Zhang ${ }^{33}$

\section{(DES Collaboration)}

${ }^{1}$ Department of Physics and Astronomy, University of Pennsylvania, Philadelphia, PA 19104, USA

${ }^{2}$ Kavli Institute for Cosmological Physics, University of Chicago, Chicago, IL 60637, USA

${ }^{3}$ Department of Astronomy and Astrophysics, University of Chicago, Chicago, IL 60637, USA

${ }^{4}$ School of Mathematics and Physics, University of Queensland, Brisbane, QLD 4072, Australia

${ }^{5}$ Lawrence Berkeley National Laboratory, 1 Cyclotron Road, Berkeley, CA 94720, USA

${ }^{6}$ The Research School of Astronomy and Astrophysics, Australian National University, ACT 2601, Australia

${ }^{7}$ Institute of Cosmology and Gravitation, University of Portsmouth, Portsmouth, PO1 3FX, UK

${ }^{8}$ ARC Centre of Excellence for All-sky Astrophysics (CAASTRO), Australia

${ }^{9}$ School of Physics and Astronomy, University of Southampton, Southampton, SO17 1BJ, UK

${ }^{10}$ University of Copenhagen, Dark Cosmology Centre, Juliane Maries Vej 30, 2100 Copenhagen O, Denmark

${ }^{11}$ Korea Astronomy and Space Science Institute, Yuseong-gu, Daejeon, 305-348, Republic of Korea

${ }_{13}^{12}$ Harvard-Smithsonian Center for Astrophysics, 60 Garden Street, Cambridge, MA 02138, USA

${ }^{13}$ African Institute for Mathematical Sciences, 6 Melrose Road, Muizenberg, 7945, South Africa

${ }^{14}$ South African Astronomical Observatory, P.O. Box 9, Observatory 7935, South Africa

${ }^{15}$ George P. and Cynthia Woods Mitchell Institute for Fundamental Physics and Astronomy, and Department of Physics and Astronomy, Texas A\&M University, College Station, TX 77843, USA

${ }^{16}$ INAF, Astrophysical Observatory of Turin, I-10025 Pino Torinese, Italy

${ }^{17}$ Millennium Institute of Astrophysics and Department of Physics and Astronomy, Universidad Católica de Chile, Santiago, Chile

${ }^{18}$ Department of Astronomy, University of California, Berkeley, CA 94720-3411, USA

${ }^{19}$ Miller Senior Fellow, Miller Institute for Basic Research in Science, University of California, Berkeley, CA 94720, USA

${ }^{20}$ Santa Cruz Institute for Particle Physics, Santa Cruz, CA 95064, USA

${ }_{21}^{21}$ PITT PACC, Department of Physics and Astronomy, University of Pittsburgh, Pittsburgh, PA 15260, USA

${ }^{22}$ Centre for Astrophysics \& Supercomputing, Swinburne University of Technology, Victoria 3122, Australia

${ }^{23}$ Department of Physics, University of Namibia, 340 Mandume Ndemufayo Avenue, Pionierspark, Windhoek, Namibia

${ }^{24}$ Harvard-Smithsonian Center for Astrophysics, 60 Garden Street, Cambridge, MA 02138, USA

${ }_{25}$ Gordon and Betty Moore Foundation, 1661 Page Mill Road, Palo Alto, CA 94304, USA

${ }^{26}$ Australian Astronomical Optics, Macquarie University, North Ryde, NSW 2113, Australia

${ }^{27}$ Argonne National Laboratory, 9700 South Cass Avenue, Lemont, IL 60439, USA

${ }^{28}$ Sydney Institute for Astronomy, School of Physics, A28, The University of Sydney, NSW 2006, Australia

${ }^{29}$ Institute of Astronomy and Kavli Institute for Cosmology, Madingley Road, Cambridge, CB3 OHA, UK

${ }^{30}$ Department of Astronomy/Steward Observatory, 933 North Cherry Avenue, Tucson, AZ 85721-0065, USA

${ }_{31}$ National Center for Supercomputing Applications, 1205 West Clark Street, Urbana, IL 61801, USA

${ }^{32}$ Institute of Astronomy, University of Cambridge, Madingley Road, Cambridge CB3 OHA, UK

${ }^{33}$ Fermi National Accelerator Laboratory, P.O. Box 500, Batavia, IL 60510, USA

${ }^{34}$ Division of Theoretical Astronomy, National Astronomical Observatory of Japan, 2-21-1 Osawa, Mitaka, Tokyo 181-8588, Japan

${ }^{5}$ Institute of Astronomy and Astrophysics, Academia Sinica, Taipei 10617, Taiwan

${ }^{36}$ Observatories of the Carnegie Institution for Science, 813 Santa Barbara Street, Pasadena, CA 91101, USA

${ }^{37}$ Cerro Tololo Inter-American Observatory, National Optical Astronomy Observatory, Casilla 603, La Serena, Chile ${ }^{38}$ LSST, 933 North Cherry Avenue, Tucson, AZ 85721, USA

${ }^{39}$ CNRS, UMR 7095, Institut d'Astrophysique de Paris, F-75014, Paris, France

${ }^{40}$ Sorbonne Universités, UPMC Univ Paris 06, UMR 7095, Institut d'Astrophysique de Paris, F-75014, Paris, France

${ }^{41}$ Department of Physics \& Astronomy, University College London, Gower Street, London, WC1E 6BT, UK

${ }^{42}$ Kavli Institute for Particle Astrophysics \& Cosmology, P.O. Box 2450, Stanford University, Stanford, CA 94305, USA

${ }^{43}$ SLAC National Accelerator Laboratory, Menlo Park, CA 94025, USA

${ }^{44}$ Centro de Investigaciones Energéticas, Medioambientales y Tecnológicas (CIEMAT), Madrid, Spain 


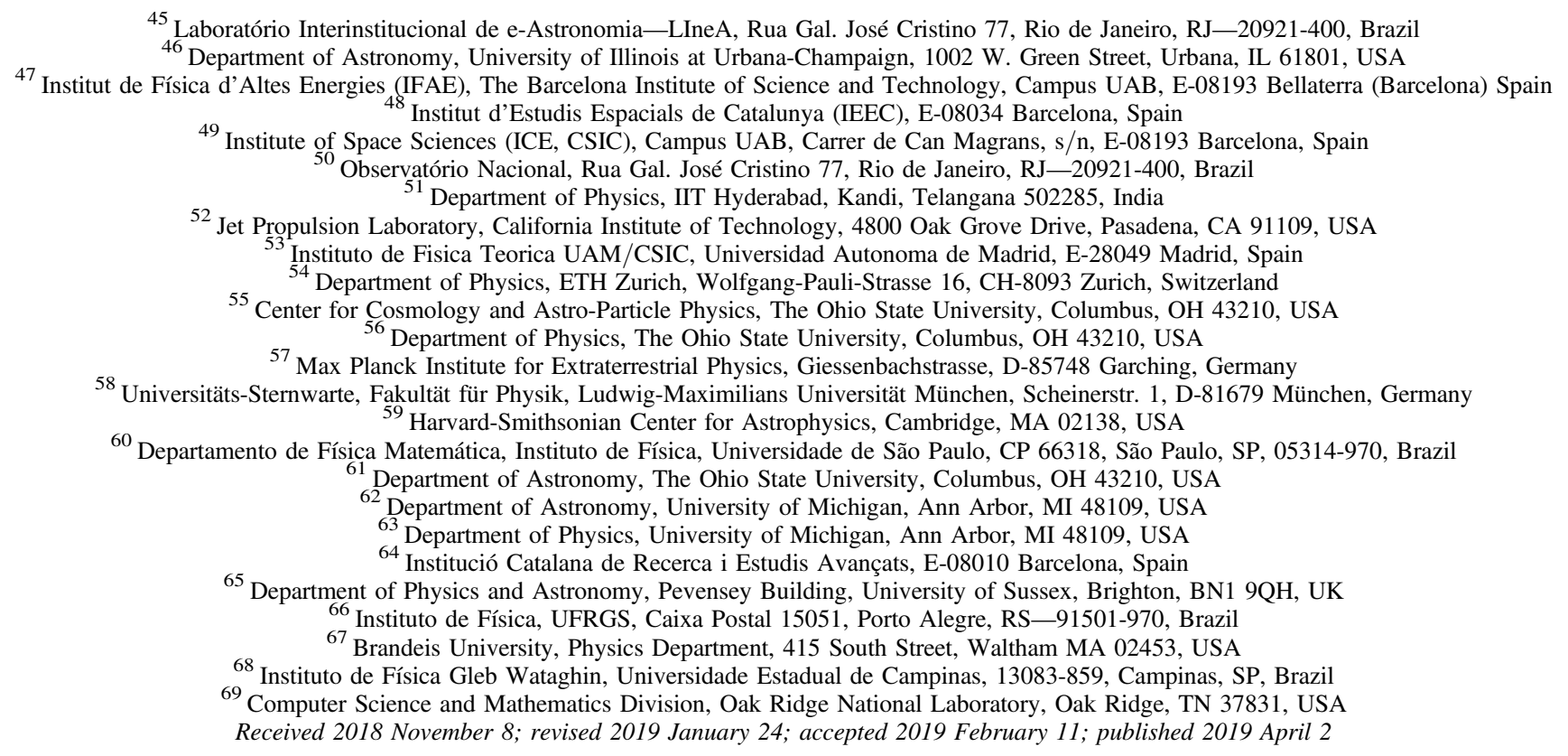

\begin{abstract}
We present the analysis underpinning the measurement of cosmological parameters from 207 spectroscopically classified SNe Ia from the first 3 years of the Dark Energy Survey Supernova Program (DES-SN), spanning a redshift range of $0.017<z<0.849$. We combine the DES-SN sample with an external sample of 122 low-redshift $(z<0.1)$ SNe Ia, resulting in a "DES-SN3YR" sample of 329 SNe Ia. Our cosmological analyses are blinded: after combining our DES-SN3YR distances with constraints from the Cosmic Microwave Background, our uncertainties in the measurement of the dark energy equation-of-state parameter, $w$, are 0.042 (stat) and 0.059 (stat+syst) at $68 \%$ confidence. We provide a detailed systematic uncertainty budget, which has nearly equal contributions from photometric calibration, astrophysical bias corrections, and instrumental bias corrections. We also include several new sources of systematic uncertainty. While our sample is less than one-third the size of the Pantheon sample, our constraints on $w$ are only larger by $1.4 \times$, showing the impact of the DES-SN Ia light-curve quality. We find that the traditional stretch and color standardization parameters of the DES-SNe Ia are in agreement with earlier SN Ia samples such as Pan-STARRS1 and the Supernova Legacy Survey. However, we find smaller intrinsic scatter about the Hubble diagram $(0.077 \mathrm{mag})$. Interestingly, we find no evidence for a Hubble residual step $(0.007 \pm 0.018 \mathrm{mag})$ as a function of host-galaxy mass for the DES subset, in $2.4 \sigma$ tension with previous measurements. We also present novel validation methods of our sample using simulated SNe Ia inserted in DECam images and using large catalog-level simulations to test for biases in our analysis pipelines.
\end{abstract}

Key words: cosmological parameters - dark energy - supernovae: general

Supporting material: machine-readable table

\section{Introduction}

The discovery of the accelerating expansion of the universe (Riess et al. 1998; Perlmutter et al. 1999) has motivated an era of cosmology surveys with the goal of measuring the mysterious properties of dark energy. The use of standardizable $\mathrm{SNe}$ Ia to measure distances has proven to be a vital tool in constraining the nature of dark energy because they probe the geometry of the universe throughout a large portion of cosmic time.

The Dark Energy Survey Supernova Program (hereafter DES-SN) has found thousands of photometrically classified $\mathrm{SNe}$ Ia at redshifts from $0.01<z<1.2$ using repeated observations in the southern celestial hemisphere searching over an area of $27 \mathrm{deg}^{2}$ (Bernstein et al. 2012). Over the full 5 years of the survey, DES-SN is expected to obtain the largest single data set of photometrically classified $\mathrm{SNe}$ Ia to date. DES-SN has spectroscopically confirmed a subset of $\sim 500$
SNe Ia at redshifts from $0.017<z<0.849$. In this work, we analyze the first 3 years of spectroscopically confirmed SNe Ia and combine our data set with an external low-redshift SN Ia sample. This combined sample is hereafter called DESSN3YR. The subset of DES-SNe Ia is hereafter denoted "the DES subset" and the subset of low- $z$ SNe Ia from CfA3, CfA4, and CSP-1 is hereafter denoted "the low- $z$ subset" (CfA3-4; Hicken et al. 2009a, 2012; CSP-1, Contreras et al. 2010).

Over the past 2 decades, there have been three parallel and overlapping major developments in using $\mathrm{SNe}$ Ia to measure cosmological parameters, upon which the DES-SN has made improvements. The first development is the order-of-magnitude growth in the number of spectroscopically confirmed SNe Ia. Original data sets at low-redshift had tens of SNe Ia (e.g., CfA1CfA2, Riess et al. 1999; Jha et al. 2006) and the next generation of low-redshift and high-redshift data sets had hundreds of SNe Ia (e.g., CfA3-4; CSP-1; ESSENCE: Narayan et al. 2016; SDSS-II: Frieman et al. 2008; Sako et al. 2018; SNLS: Guy et al. 2010; 
PS1: Rest et al. 2014; Scolnic et al. 2018). Today, with the addition of DES-SN, there are now more than 1500 spectroscopically confirmed $\mathrm{SNe}$ Ia in total.

The second development has been in detector sensitivity, which has resulted in improved light-curve quality and distance measurement uncertainties. The 570 megapixel Dark Energy Camera (DECam; Flaugher et al. 2015), with its fully depleted CCDs and excellent $z$-band response, facilitates well-measured optical light curves at high redshift (Diehl et al. 2014).

The third major development has been the increasingly sophisticated analyses of the samples. As SN Ia data sets grow in size, analyses are better able to characterize SN Ia populations and expected biases from observational selection and analysis requirements. Improvements in the analysis over the last decade have included scene modeling photometry (SMP Holtzman et al. 2008; Astier et al. 2013, B18-SMP: Brout et al. 2018-SMP) instead of classical template subtraction, the modeling and correction of expected biases using large simulations (Kessler et al. 2009a; Perrett et al. 2010; Betoule et al. 2014), and measuring filter transmissions to achieve sub $1 \%$ calibration uncertainty (Astier et al. 2006; Doi et al. 2010; Tonry et al. 2012; Marshall et al. 2013; Burke et al. 2018). Recent cosmological parameter analyses (B14: Betoule et al. 2014, S18: Scolnic et al. 2018) have found that systematic uncertainties are roughly equal to the statistical uncertainties; this is due to the improving ability to understand and reduce systematic uncertainties with larger samples and reduced statistical uncertainties. Each new cosmology analysis (Wood-Vasey et al. 2007; Kessler et al. 2009a; Sullivan et al. 2011, B14, S14: Scolnic et al. 2014, S18, Jones et al. 2018) has built on previous analyses in their treatment of systematic uncertainties. Here we continue in this tradition of improvements and also study several previously uninvestigated sources of uncertainty.

Improvement in understanding of systematic uncertainties is crucial to taking advantage of the order of magnitude increases in statistics expected in the coming years. From DES-SN alone, there is the full sample of $\sim 2000$ photometrically classified SNe Ia. Additionally, the next generation of photometric transient surveys (LSST: Ivezic et al. 2008; LSST Science Collaboration et al. 2009; WFIRST: Hounsell et al. 2018) expects tens to hundreds of thousands of photometrically classified SNe Ia.

The key analysis steps to produce cosmological constraints from our spectroscopically confirmed data set are (1) absolute calibration of the DES-SN photometric system, (2) precision photometry for light-curve fluxes, (3) simulation of large samples to predict biases, (4) light-curve fits to standardize the SN brightness and measure luminosity distance, (5) construction of bias-corrected Hubble diagram, (6) construction of full statistical and systematic covariance matrix, and (7) cosmological parameter fits. Step 1 (Burke et al. 2018; Lasker et al. 2019), step 2 (B18-SMP), and step 3 (Kessler et al. 2019) are discussed in detail in companion papers, and they are discussed within this paper in the context of understanding systematic uncertainties. Steps 4-7 are described here in detail.

There are two main results of this paper. First we present the nuisance parameters involved in the standardization of SNe Ia. Historically $\alpha$ and $\beta$, the correlation coefficients for stretch and color of supernova light curves respectively, have been used to standardize SN Ia luminosities, and $\sigma_{\text {int }}$ has been used to characterize the scatter in $\mathrm{SN}$ Ia luminosities that is not covered
The Dataset

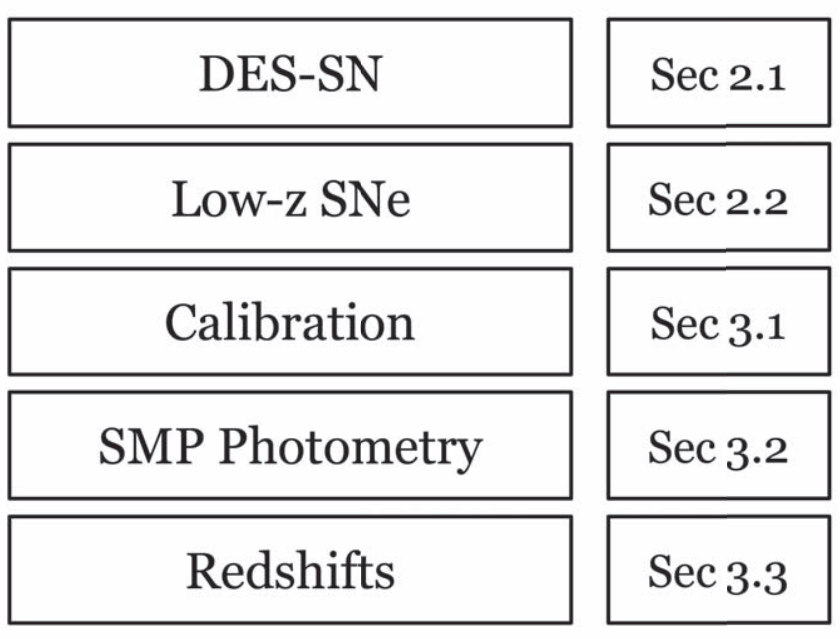

Making the Hubble Diagram

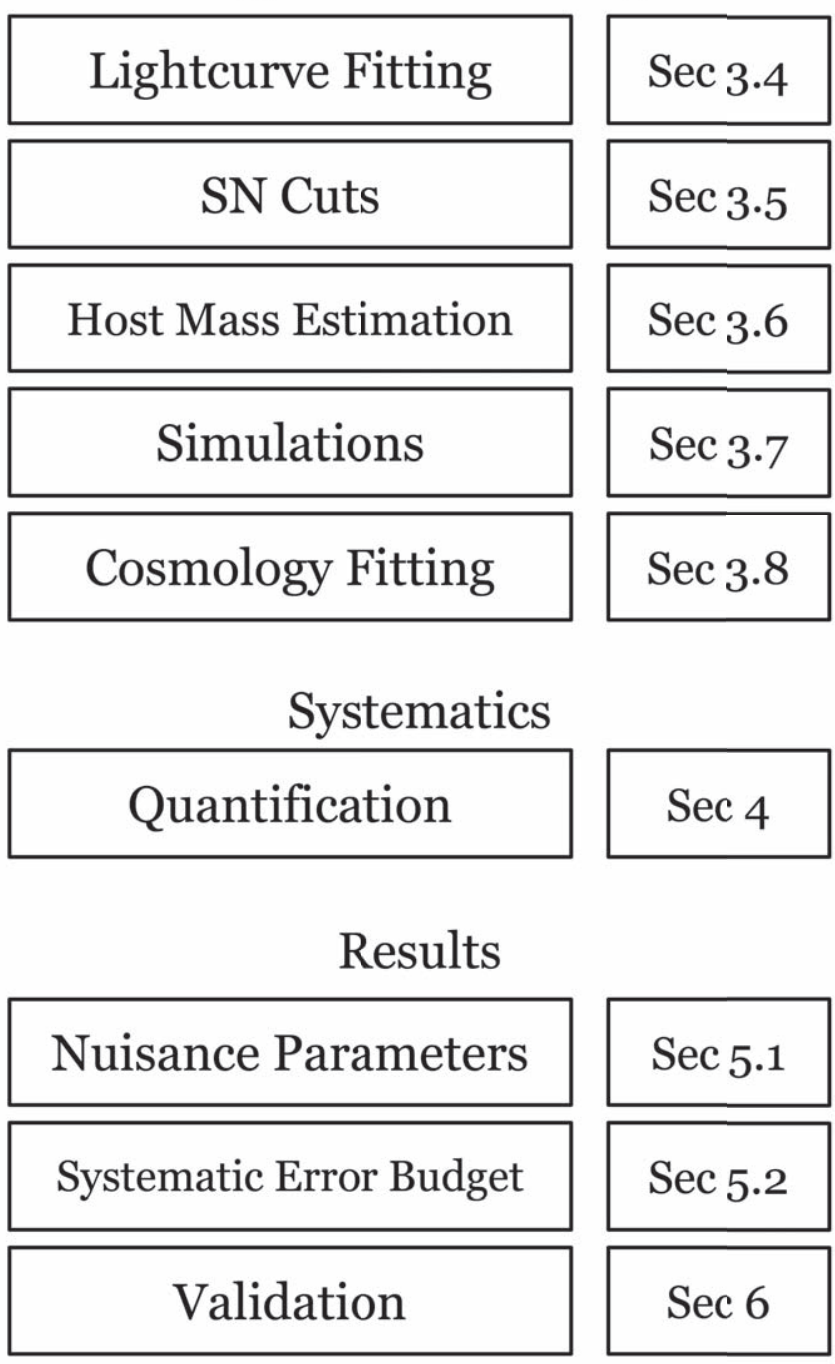

Figure 1. Analysis flowchart of this paper. Nuisance parameters, the systematic error budget, and the results of validation are considered the "Results" of this work (Section 5), and the unblinded cosmological parameter best-fit values are presented in DES Collaboration et al. (2018). 
by the measurement uncertainties. Additionally, several groups in the last decade have shown that more massive galaxies tend to host overluminous SNe Ia after color and stretch brightness standardization, suggesting improved standardizability of the SNe Ia population (Kelly et al. 2010; Lampeitl et al. 2010; Sullivan et al. 2010). This effect has been characterized as a step function in Hubble diagram residuals $(\gamma)$ across $10^{10} \mathcal{M}_{\odot}$. However, the size of this effect has been seen to vary in different samples and the physical interpretation is not understood. In this paper we discuss our own findings for these nuisance parameters using DES-SN3YR. The second main result is the statistical and systematic uncertainty budget from our $w \mathrm{CDM}$ cosmological analysis after combining with Planck Collaboration (2016) cosmic microwave background (CMB) priors. Using the analysis and results derived here, cosmological parameter constraints are shown in DES Collaboration et al. (2018).

In order to improve upon the treatment and validation of systematic uncertainties from past analyses, we use two types of SN Ia simulations to examine biases in our pipelines and to provide crosschecks of our analysis. The first set of simulations includes hundreds of catalog-level simulations with input sources of systematic uncertainty. We analyze the catalog-level simulations with steps 3-7 provided to verify our analysis pipeline and reported statistical and systematic uncertainties. These simulations are generated by the SuperNova ANAlysis software package $^{70}$ (SNANA: Kessler et al. 2009b), which has been used extensively by previous analyses to quantify expected biases and offers the capability of parallelization for generating and analyzing large simulations of SNe Ia.

For the second set of validation simulations, we generate 100,000 artificial supernova light curves that are inserted as point sources onto DECam images (hereafter "fakes"). Previous analyses have used artificial point sources to understand photometric uncertainties (Holtzman et al. 2008; Perrett et al. 2010). In DES-SN, fake supernovae light curves are used for several reasons. Fakes are used to check for biases in photometry (B18-SMP) and in the determination of $\mathrm{SN}$ Ia detection efficiency as a function of signal-to-noise $(\mathrm{S} / \mathrm{N}$; Kessler et al. 2019), thereby modeling subtle pipeline features that cannot be computed from first principles. Additionally, we present a cosmological analysis of 10,000 fake supernovae that have been recovered by the search pipeline, processed by the photometric pipeline, and processed through our cosmological analysis pipeline in the same manner as the real data set. This crosscheck is sensitive to potential unmodeled biases in the image-processing pipelines and their propagation to cosmological distance and cosmological parameter biases.

Unfortunately, neither of the methods provided address the systematic uncertainty due to calibration. To address calibration uncertainties, we compare our absolute calibration with that of the Pan-STARRS survey (Tonry et al. 2012) and SuperCal (Scolnic et al. 2015).

The organization of this paper is depicted in Figure 1 and is described as follows. In Section 2 we introduce the data samples, a combination of high-redshift SNe Ia from DES-SN and low-redshift SNe Ia from CfA and CSP-1. In Section 3 we discuss analysis procedures and characterize systematic uncertainties. In Section 4 we quantify each source of systematic uncertainty. In Section 5 we present results for the

\footnotetext{
${ }^{70}$ http://snana.uchicago.edu/
}

nuisance parameters, the systematic uncertainty budget, and the total statistical and systematic uncertainty. In Section 6 we describe our validation methods. In Section 7 we discuss a Bayesian Hierarchical method under development and its performance on validation and the DES-SN3YR sample. In Section 8 we discuss our findings, and in Section 9 we conclude.

\section{Data Sets}

\subsection{The Dark Energy Survey Supernova Program}

DES-SN performed a deep, time-domain survey in four optical bands $(g, r, i, z)$ covering $\sim 27 \mathrm{deg}^{2}$ over five seasons (2013-2018) using the DECam mounted on the $4 \mathrm{~m}$ Blanco telescope at the Cerro Tololo Inter-American Observatory (CTIO). Exposure processing (Morganson et al. 2018), difference imaging (DiffImg: Kessler et al. 2015), and automated rejection of subtraction artifacts (Goldstein et al. 2015) are run on a nightly basis. DES-SN observed in eight "shallow" fields $(\mathrm{C} 1, \mathrm{C} 2, \mathrm{X} 1, \mathrm{X} 2, \mathrm{E} 1, \mathrm{E} 2, \mathrm{~S} 1, \mathrm{~S} 2)$ with single-epoch $50 \%$ completeness depth of $23.5 \mathrm{mag}$ and in two "deep" fields (C3, X3) with depth $\sim 24.5$ mag in all four bands. The 10 DES-SN fields are grouped into four separated regions $(C, X, E, S)$, where each group contains adjacent pointings on the sky. For example, $\mathrm{C} 1, \mathrm{C} 2, \mathrm{C} 3$ are adjacent fields denoted group C, where each field center is separated by $2^{\circ}$. Tables 1 and 2 of Kessler et al. (2015) contain detailed information of the DES-SN observing fields.

For a SN to be considered a "candidate," we require two detections at the same location on two separate nights in any of the four bands. A subset of the candidates are selected for spectroscopic follow-up to obtain a type classification and redshift. A detailed overview of the spectroscopic follow-up program as well as a general overview of the DES-SN program and observing strategy can be found in D'Andrea et al. (2018).

Over the first 3 years of DES-SN, from 2013 September to 2016 February, we discovered roughly $\sim 12,000$ candidates of which $\sim 2000$ are likely SNe Ia. In this first analysis we analyze only the spectroscopically confirmed SN Ia subset of the data. As described in D'Andrea et al. (2018), 307 transients of the likely SNe Ia were targeted for spectroscopic classification using a variety of spectroscopic resources, and 251 were confirmed as Type Ia over a redshift range of $0.017<z<0.849$. The majority of spectra come from the Anglo-Australian Telescope (AAT) as part of the OzDES program (Yuan et al. 2015; Childress et al. 2017). The distribution of redshifts for the spectroscopically confirmed SNe Ia from the first $3 \mathrm{yr}$ of DESSN observations is shown in Figure 2. DES-SN SNe at lower redshift are preferentially cut from the sample used for cosmological analysis due to poor light-curve coverage and light-curve fit quality. Quality cuts and selection requirements are discussed in detail in Section 3.5. The [min, mean, max] redshifts after performing the data selection cuts are $[0.08,0.39$, $0.85]$, respectively.

Additional data are acquired using an in situ calibration process called "DECal" (Marshall et al. 2013). The Blanco/DECam optical system and filter transmission functions are measured under multi-wavelength illumination. DES-SN also acquires real-time meteorological data using the SUOMINET system ${ }^{71}$ to track precipitable water vapor levels and auxiliary "aTmCAM"

\footnotetext{
71 https://www.suominet.ucar.edu/
} 


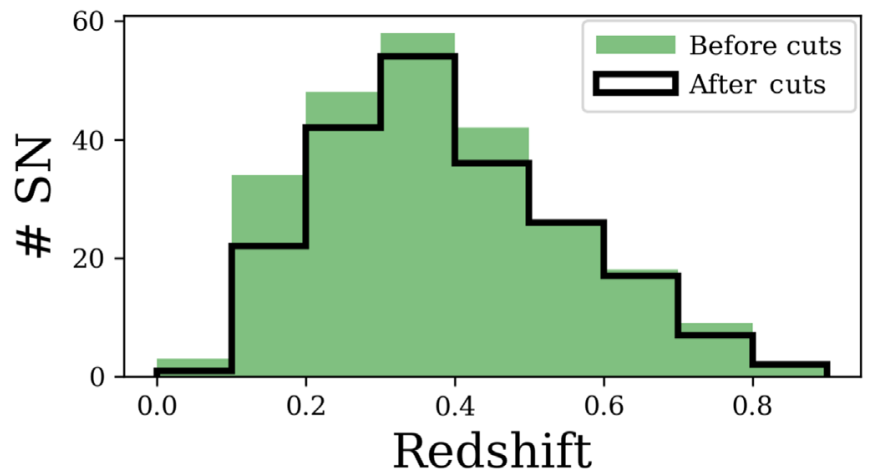

Figure 2. Histogram of the 251 spectroscopically confirmed SNe Ia is shown in green. The sub-sample of SNe Ia used for cosmological parameter analysis that pass all quality cuts is shown in black.

instrumentation ( $\mathrm{Li}$ et al. 2014) to measure atmospheric conditions.

\subsection{External Low-redshift Samples}

Cosmological constraints from SNe Ia are best obtained with samples at both low-redshift and high-redshift. We utilize four publicly available low-redshift surveys: CfA3S, CfA3K, CfA4, and CSP-1 (Jha et al. 2006; Hicken et al. 2009a, 2012; Contreras et al. 2010), consisting of 303 spectroscopically confirmed SNe Ia in the redshift range $0.01<z<0.1$. These low-redshift surveys are chosen because of their well-defined calibrations. B14 and S18 included 22 SNe Ia from CfA 1 and CfA2 as part of their analyses. However, we chose not to include them in our analysis because filter transmission functions were not provided for those samples.

\section{Analysis}

Here we describe the analysis procedures used to measure cosmological parameters. The majority of this section describes the analysis of the DES subset itself, though we also include our analysis of the low-redshift sample. The description of systematic uncertainties associated with each step in the analysis is laid out in this section, and each source of systematic uncertainty is quantified in Section 4. We rely on complementary work in Kessler et al. (2019), hereafter K19, which details the simulations of DES-SN3YR. These simulations are used for computing bias corrections in Section 3.7.

\subsection{Calibration}

SN Ia cosmological constraints rely on the ability to internally transform each $\mathrm{SN}$ flux measurement in ADU (Analog/Digital Units) into a "top-of-the-galaxy" brightness. This is done in two steps, first via measurements of Hubble Space Telescope (HST) CalSpec ${ }^{72}$ standard stars to obtain a top-of-the-atmosphere brightness, which is discussed here. Second, we obtain top-of-the-galaxy brightness by accounting for the Milky Way (MW) extinction along the line of sight, values for which are obtained from Schlegel et al. (1998) and Schlafly \& Finkbeiner (2011). Measurements of cosmological parameters using SNe Ia are sensitive to filter calibration uncertainties (internal) due to the fact that at higher redshift, constraints of the SN light-curve models rely on observed fluxes in a different set of filters than at lower redshift. A

\footnotetext{
${ }^{72}$ http://www.stsci.edu/hst/observatory/crds/calspec.html
}

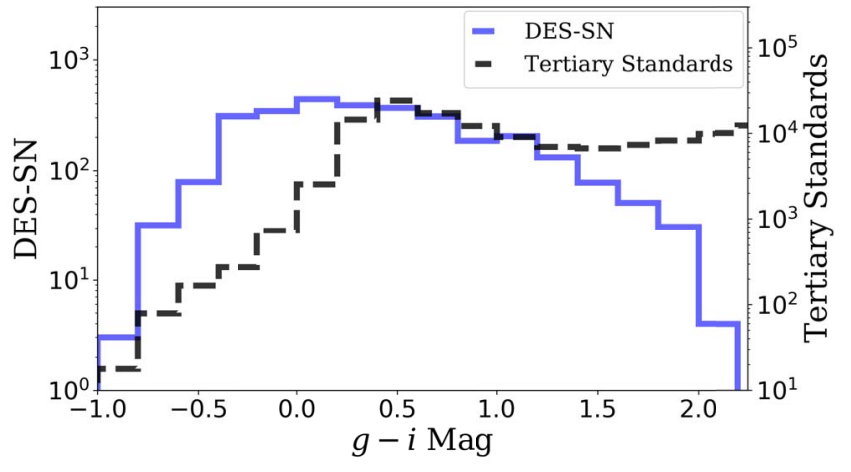

Figure 3. Blue: distribution of observed $g-i$ colors for the DES-SN sample observations. Epochs with $\mathrm{S} / \mathrm{N}>10$ are shown. Black: distribution of $g-i$ colors for the tertiary standard stars used for internal calibration. The validity of chromatic corrections is evaluated over the stellar color range (black), but the corrections are applied to the DES-SN fluxes (blue).

dependence in SN cosmological distances as a function of redshift could arise from differences in the calibration between the low- $z$ and DES subsets (external). Herein we discuss the steps taken to both internally and externally calibrate the DESSN measurements.

\subsubsection{Star Catalog}

Here we describe the process of calibrating each of the DESSN images. Photometry of approximately 50 tertiary standard stars are used to determine a zero-point for each DECam CCD image. The catalog of tens of thousands of tertiary star magnitudes is described in Burke et al. (2018). These stars are internally calibrated using a "Forward Global Calibration Method" (FGCM) to an rms of 6 mmag. FGCM models the rate of photons detected by the camera by utilizing measurements of instrument transmission, atmospheric properties, a model of the atmosphere, and a model of the source. Spectral Energy Distribution (SED)-dependent chromatic corrections are applied to the standard stars that extend the $6 \mathrm{mmag}$ calibration uncertainty to be valid over a very wide color range $(-1<g-i<3)$. The $g-i$ color distribution of the tertiary standard stars is shown in Figure 3. The color distribution of the DES subset light curves is different from that of the standard stars and is discussed in Section 3.2.

\subsubsection{AB Offsets}

The FGCM catalog is calibrated to the AB system (Oke \& Gunn 1983) using measurements of the HST CalSpec standard C26202. As detailed in Burke et al. (2018), we compute synthetic magnitudes of C26202 by multiplying the CalSpec spectrum with the standard instrumental and atmospheric passbands used in the FGCM calibration ${ }^{73}$ DECam filter transmission functions. The synthetic magnitudes are compared to the FGCM catalog magnitudes of C26202 for each passband, and the magnitude difference is applied to the FGCM catalog so that the observed and synthetic magnitudes of the standard are in perfect agreement. C26202 was chosen because it is located in "C3," which is one of the deep fields and has been observed more than 100 times during the course of the survey. C26202 is sufficiently faint to avoid saturation and is observed in a similar range of seeing conditions as that of the DES-SN

$\overline{73}$ Y3A1 passbands from Burke et al. (2018). 

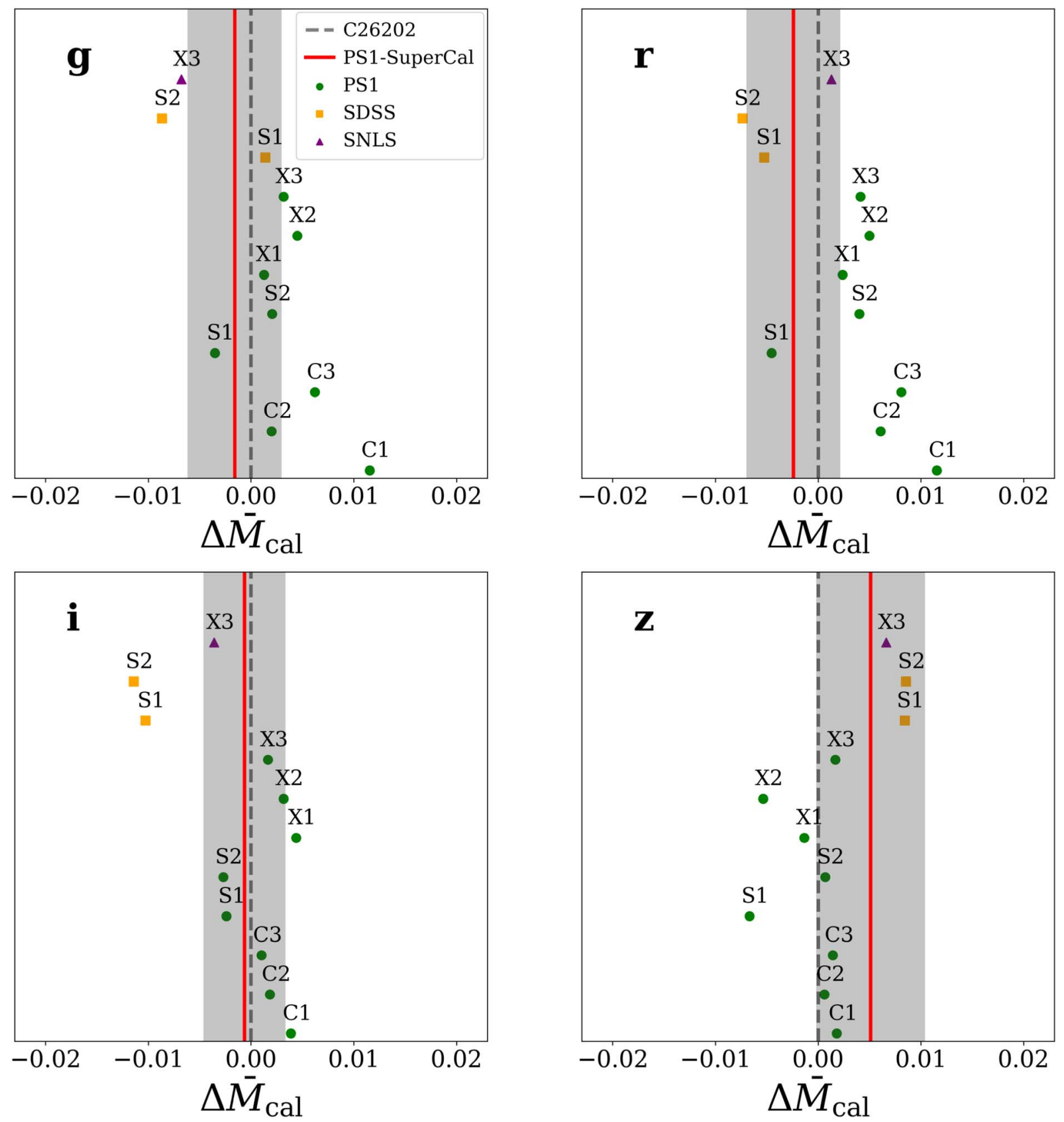

Figure 4. Relative offsets in stellar magnitudes when comparing PS1, SDSS, and SNLS to overlapping DES fields ( $\left.\Delta \bar{M}_{\text {cal }}\right)$. Offsets are further broken down by field. In each panel, $\Delta \bar{M}_{\text {cal }}$ for the HST Calspec magnitude of C26202 is defined to be zero. Each of the points are determined from a comparison of DECam and external survey photometry accounting for difference in filter transmission functions. SNLS and SDSS are shown for reference; however, it is only PS1 that is used to determine the goodness of the calibration. The vertical red line is the mean of the PS1-DES overlap (green points) shifted by the PS1 offset to SuperCal. The gray area represents the quadrature sum of the uniformity uncertainty and the SuperCal uncertainty in absolute calibration (Section 4.1).

data set. Other CapSpec standards in the DES footprint are either saturated or were observed with short exposures under twilight conditions. We do not find any dependence in the corrected, top-of-the-atmosphere fluxes of C26202 on airmass, sky brightness, CCD number of the observation, or exposure time.

A secondary method of calibrating the FGCM catalog is to cross-calibrate with catalogs from other surveys that are also tied to the $\mathrm{AB}$ system. Using tertiary standard stars in 8 of the 10 of the DES-SN fields (DES Fields: C1, C2, C3, S1, S2, X1,
$\mathrm{X} 2, \mathrm{X} 3$ ) that overlap with the footprint of other surveys, we measure the calibrated brightness differences for stars observed by both surveys and compare these differences to predictions using a spectral library and known filter transmissions. We define $\Delta M_{\mathrm{cal}}$ as the offset between the predicted and observed brightness differences for stars with the same color as the Calspec standard C26202. In Figure 4, we examine the mean difference $\left(\Delta \bar{M}_{\text {cal }}\right)$ for several groupings of overlapping calibration stars. For comparison, we examine the agreement between DES and PS1 (green), DES and SDSS (orange), and 
DES and SNLS (violet). We also define PS1-SuperCal (red) as the agreement between DES and PS1, if the absolute calibration of PS1 were shifted by the weighted average of differences among the PS1, SDSS, and SNLS calibration (see Scolnic et al. 2015 for explanation).

Burke et al. (2018) apply FGCM to the DECam images and achieve a calibration uniformity across the sky of $\sim 6$ mmag. As a crosscheck for our SN fields, we quantify the relative consistency the DES-SN fields from the standard deviation of PS1-DES $\Delta M_{\text {cal }}$, which is $4.1,4.3,2.5,3.1$ mmags in the $g, r, i$, $z$ bands, respectively. The observed consistency between PS1 and DES is $2-4 \mathrm{mmag}$, which shows that $\sim 6 \mathrm{mmag}$ is a conservative estimate of the relative calibration uncertainties due to non-uniformity. Lastly, the observed offsets of stellar magnitudes among PS1, SDSS, and SNLS shown in Figure 4 are consistent with the scatter seen in Scolnic et al. (2015); these differences are shown for reference and are not used in this analysis.

\subsection{SN Photometry}

The light curves used in this analysis are provided by B18SMP, which measures SN brightnesses by adopting a scene modeling approach. In SMP, a variable transient flux and temporally constant host galaxy are forward modeled simultaneously. B18-SMP test the accuracy of the SMP pipeline by processing a sample of 10,000 realistic SNe Ia light curves that were injected as point sources onto DECam images ("fake $\mathrm{SNe}$ "). Upon comparison of input and measured fake $\mathrm{SNe}$ fluxes, B18-SMP find that biases in the photometric pipeline are limited to $3 \mathrm{mmag}$ (see Figure 3 of B18-SMP).

Analyzing fakes near bright galaxies, B18-SMP also find that the photometric scatter increases with the local surface brightness (denoted "the host SB dependence"). This increase is similar to what was observed in DiffImg (Kessler et al. 2015). The host SB dependence is accounted for by scaling our photometric uncertainties of fake $\mathrm{SNe}$ near bright host galaxies to match the observed scatter in SMP flux residuals. This scaling is determined as a function of host-galaxy surface brightness $\left(m_{\mathrm{SB}}\right)$,

$$
\hat{S}_{\mathrm{SMP}}\left(m_{\mathrm{SB}}\right)=\frac{\operatorname{rms}\left[\left(F_{\text {true }}-F_{\mathrm{SMP}}\right) / \sigma_{\text {Ref }}\right]_{\text {fake }}}{\left\langle\sigma_{\mathrm{SMP}} / \sigma_{\text {Ref }}\right\rangle_{\text {fake }}},
$$

where rms is the root mean square in a bin of $m_{\mathrm{SB}}, \sigma_{\mathrm{SMP}}$ is the SMP flux uncertainty, \langle\rangle indicates an average in the $m_{\mathrm{SB}}$ bin, $\sigma_{\text {Ref }}$ is the calculated uncertainty based on observing conditions (zeropoint, sky noise, PSF), $F_{\mathrm{SMP}}$ is the fit flux from SMP, and $F_{\text {true }}$ is the input flux of the fake $\mathrm{SN}$. The size of $\hat{S}_{\mathrm{SMP}}\left(m_{\mathrm{SB}}\right)$ can be seen in Figure 5 of B18-SMP and can be as large as 4 at $m_{\mathrm{SB}}=21$. These corrections are applied directly to the DES-SN sample.

After SMP, there is an additional set of SED-dependent chromatic corrections made to the DES-SN Ia fluxes, similar to the corrections made to the stellar fluxes discussed in Section 3.1.1. The impact of these corrections is presented in Lasker et al. (2019) and is discussed here in Section 4.1. One potential issue is the validity of the chromatic corrections applied to the SN fluxes whose color range $(-1.0<g-i<2.2)$ is redder than that of the majority of tertiary calibration stars $(0.2<g-i<3)$ and is shown in Figure 3. For $g-i<0.2$, there is a drop-off in tertiary standard star counts as the star distribution enters the realm of blue horizontal branch stars and white dwarfs. While we do not have the statistics to
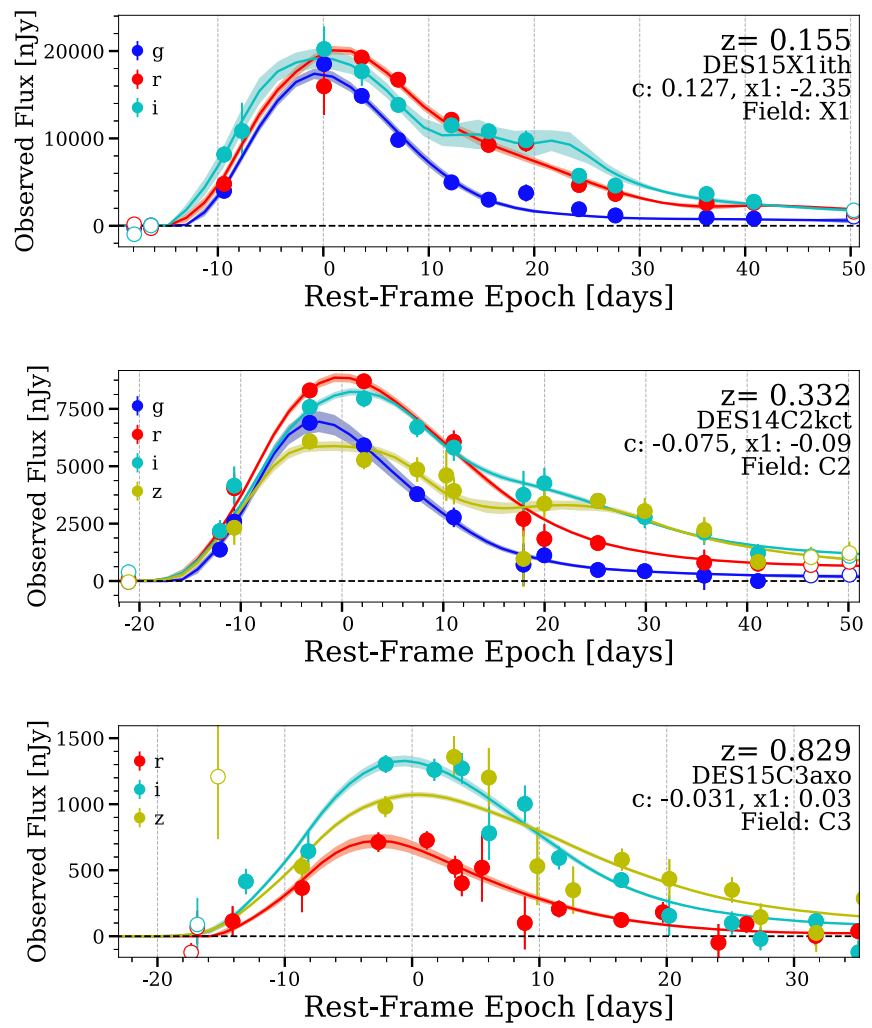

Figure 5. Representative light curves of the DES-SN3YR spectroscopic sample with photometric data determined with SMP (points). SALT2 fits to the light curve are overlaid (curves), and fitted color and stretch values are shown. There is no $g$ band in the bottom panel because $z=0.829$ is beyond the range of the B14 $g$-band model. Supernovae with C3 (or X3) in the name are found in deep fields, the remaining SNe are found in the shallow fields. Open points are excluded from the SALT2 fits.

validate the 6 mmag calibration uncertainty for the bluest stars $(-1.0<g-i<0.2)$, we assume that chromatic corrections are valid for $\mathrm{SN}$ Ia fluxes in this color range. The chromatic corrections applied to the tertiary standards in the color range of $g-i \in[0,3]$ show no significant trends at the bluest colors, and thus we have confidence in applying the corrections to the fraction of bluest $\mathrm{SN}$ Ia epochs in the color range $g-i \in[-1,0]$.

\subsection{Redshifts}

Redshifts for the DES subset are presented in D'Andrea et al. (2018). Redshifts of the low-redshift sample are obtained from their respective surveys, to which we make peculiar velocity corrections. The corrections due to coherent flows of SN host galaxies have been performed in the same manner as S18. Peculiar velocities are calculated using the matter density field calibrated by the $2 \mathrm{M}++$ catalog (Skrutskie et al. 2006) out to $z \sim 0.05$, with a light-to-matter bias of $\beta=0.43$ and a dipole as described in Carrick et al. (2015). We adopt the error in peculiar velocity correction of $250 \mathrm{~km} \mathrm{~s}^{-1} \mathrm{Mpc}^{-1}$ motivated by dark matter simulations of Carrick et al. (2015) as well as from the comparison of low-redshift and intermediate-redshift $\mathrm{SNe}$ scatter described in S18.

The redshifts of host galaxies used in this analysis are typically reported with an accuracy of $\sim 10^{-4}$ for low$z$ and to $\sim 5 \times 10^{-4}$ for intermediate redshift. For $71 \mathrm{SNe}$ in the DES subset, a host-galaxy redshift was not obtained and redshifts were determined from the $\mathrm{SN}$ spectrum, resulting in 


\section{DES Subset}

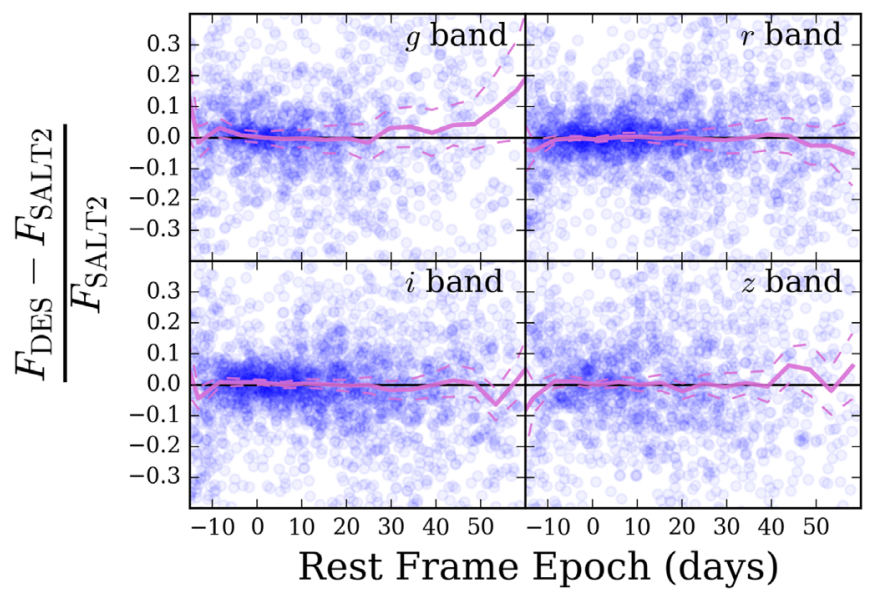

Low-z Subset

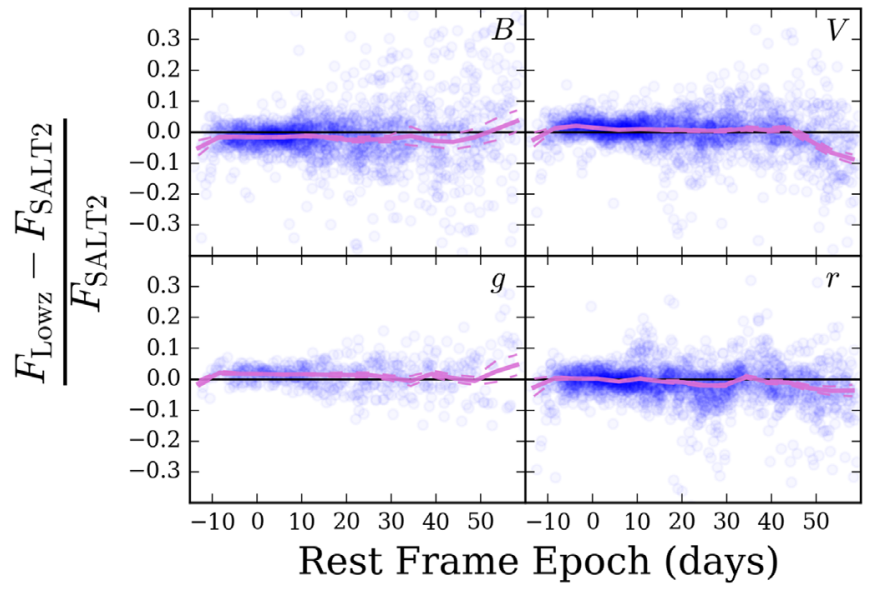

Figure 6. Fractional flux residuals to the best-fit SALT2 light-curve model. Top: the DES-SN3YR spectroscopic sample in the four DES filter bands [griz]. Bottom: the low $z$ subset where photometric observations have been grouped by filters with similar wavelength coverage $[B V g r] . F_{\mathrm{DES}}$ and $F_{\text {Low } z}$ are the SN flux from the data; $F_{\mathrm{SALT} 2}$ is the flux of the best-fit SALT2 model. The mean of each distribution is shown in solid curve, and the uncertainty on the mean is shown as dashed curves.

redshift uncertainty $\sim 5 \times 10^{-3}$. These redshift uncertainties propagate to $\mathrm{SN}$ scatter in distance. However, more important than the statistical uncertainty is the possibility of a systematic shift in redshift due to cosmological effects. A systematic shift could be caused, for example, by a gravitational redshift due to the density of our local environment (Calcino \& Davis 2017). Wojtak et al. (2015) show the expected distributions for typical environments in $\Lambda \mathrm{CDM}$ can be described by a one sigma fluctuation from the mean potential with a shift of $\Delta z \approx 2 \times 10^{-5}$.

\subsection{Light-curve Fits}

In order to obtain distance moduli $(\mu)$ from SN Ia light curves, we fit the light curves with the SALT2 model (Guy et al. 2010) using the trained model parameters from B14 over an SED wavelength range of $200-900 \mathrm{~nm}$. We select passbands whose central wavelength, $\bar{\lambda}$, satisfies $280<\bar{\lambda} /(1+z)<700 \mathrm{~nm}$, and we select epochs satisfying -15 to +45 days with respect to the epoch of peak brightness in the rest frame. We use the SNANA implementation (Kessler et al. 2009b) based on MINUIT
Table 1

\# SN after Iteratively Applied Cuts

\begin{tabular}{lrrr}
\hline \hline Requirement & $\begin{array}{r}\text { DES-SN } \\
\#[\text { Cut }]\end{array}$ & $\begin{array}{r}\text { Low- } z \\
\text { \# [Cut] }\end{array}$ & $\begin{array}{r}\text { Total SN } \\
\text { \# [Cut] }\end{array}$ \\
\hline Initial & $251^{\mathrm{a}}$ & $333^{\mathrm{b}}$ & 542 \\
\hline$z>0.01$ & $251[0]$ & $261[72]$ & $512[72]$ \\
Fit Convergence & $244[7]$ & $257[4]$ & $501[11]$ \\
$S / N>5$ in 2 bands & $239[5]$ & $250[7]$ & $439[12]$ \\
$T_{\text {rest }}>10, T_{\text {rest }}<0$ & $230[9]$ & $248[2]$ & $481[11]$ \\
$E(B-V)_{\mathrm{MW}}<0.25$ & $230[0]$ & $243[5]$ & $473[5]$ \\
$-0.3<c<0.3$ & $224[6]$ & $170[73]$ & $394[79]$ \\
$-3<x_{1}<3$ & $221[3]$ & $150[20]$ & $371[23]$ \\
$\sigma_{x_{1}}<1$ & $211[10]$ & $150[0]$ & $361[10]$ \\
$P_{\text {fit }}>0.01$ & $208[3]$ & $127[23]$ & $335[26]$ \\
Valid BiasCor & $207[1]$ & $125[2]$ & $332[3]$ \\
Chauvenets criterion & $207[0]$ & $122[3]$ & $329[3]$ \\
\hline Cosmo. Sample & 207 & 122 & 329 \\
\hline
\end{tabular}

Notes.

${ }^{\text {a }}$ Discovered by DiffImg and spectroscopically confirmed (D'Andrea et al. 2018).

${ }^{\text {b }}$ CfA3, CfA4, and CSP-1 samples.

(James \& Roos 1975), and we use the MINOS option for the fitted parameter uncertainties. A discussion about techniques used to avoid pathological fits is described in Appendix A.

Each light-curve fit determines parameters color $c$, stretch $x_{1}$, the overall amplitude $x_{0}$, with $m_{B} \equiv-2.5 \log _{10}\left(x_{0}\right)$, and time of peak brightness $t_{0}$ in the rest-frame $B$-band wavelength range. In addition, we compute light-curve fit probability $P_{\text {fit }}$, which is the probability of finding a light-curve data-model $\chi^{2}$ as large or larger assuming Gaussian-distributed flux uncertainties. In Figure 5, three representative DES-SN light curves are shown with overlaid light-curve fits using the SALT2 model. Normalized flux residuals to the SALT2 light-curve model for the DES-SN3YR sample are shown in Figure 6. Both the DES subset and low- $z$ subset SALT2 model fluxes for all restframe passbands are consistent to within $<2$ mmag. Calibration offsets to the SALT2 model are adopted as systematic uncertainty; this is described in Section 4.1. All light-curve fit parameters for the DES-SN3YR sample are publicly available in machine-readable format as described in Appendix B and Appendix C.

\subsection{Selection Requirements}

For this analysis, we require all $\mathrm{SNe}$ Ia to have adequate light-curve coverage in order to reliably constrain light-curve fit parameters, and we limit ourselves to a model-training range of SN Ia properties that limit systematic biases in the recovered distance modulus measurement. The sequential loss of SNe Ia from the sample due to cuts is shown in Table 1. We start by requiring $z>0.01$ and our light-curve fits to converge. We define $T_{\text {rest }}$ as the number of days since $t_{0}$ in the rest frame of the SN. Dai \& Wang (2016) showed that poorly sampled light curves can result in large Hubble residual outliers, even though the fit $\chi^{2}$ shows no indication of a problem. Thus, we require an observation before peak brightness $\left(T_{\text {rest }}<0\right)$, an observation at least 10 days after peak brightness $\left(T_{\text {rest }}>10\right)$, and an observation with $\mathrm{S} / \mathrm{N}>5$ in at least two bands. We require $-3<x_{1}<3$ and $-0.3<c<0.3$ over which the light-curve model has been trained (Guy et al. 2010). For the low-redshift 
DES-SN Sample Data and Simulations
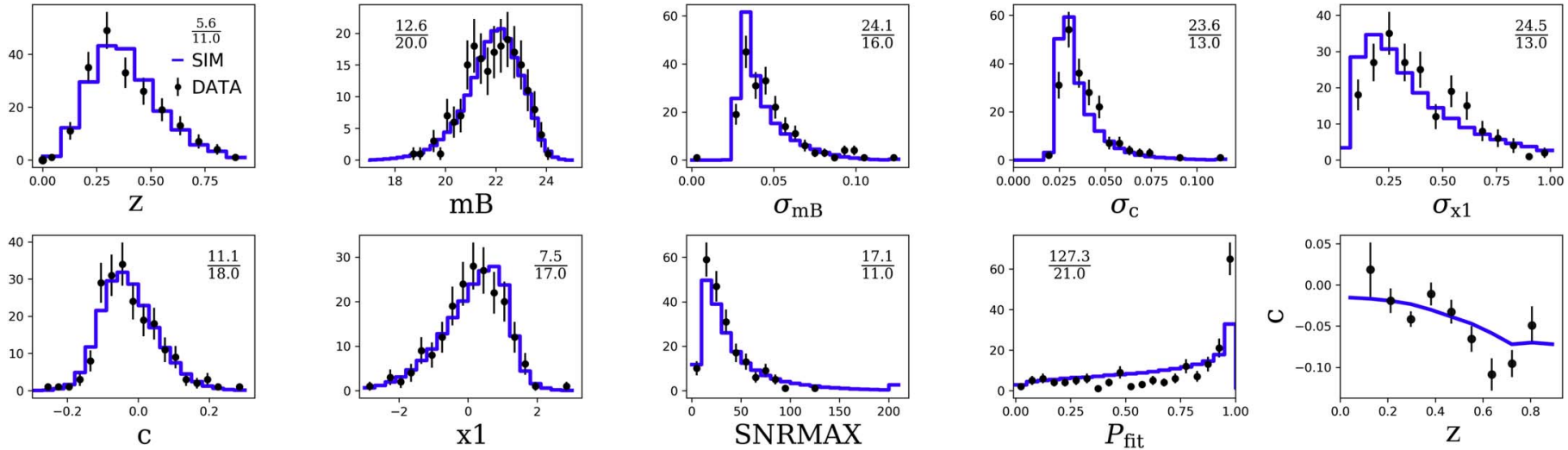

Low- $z$ Sample Data and Simulations
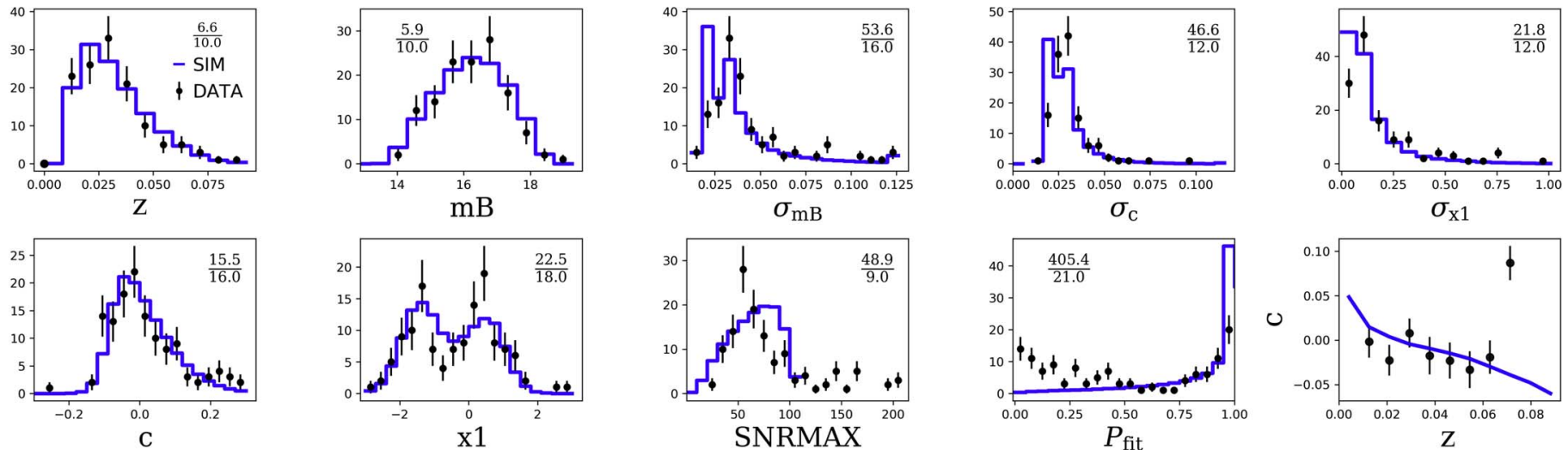

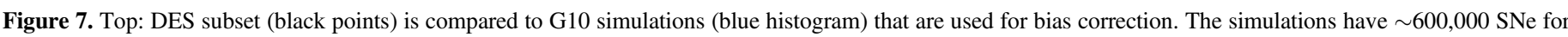

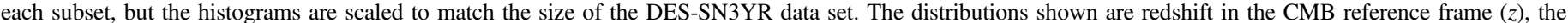

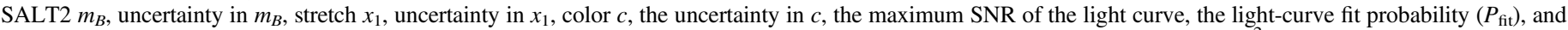
lastly $c$ as a function of redshift. Bottom: same as top but for the external low- $z$ sample. The fractions shown in each panel are $\chi^{2} /$ ndof.

samples, we require limited MW extinction following B14 and S18, $E(B-V)_{\mathrm{MW}}<0.25$. The DES-SN Fields have low MW extinction, and thus the $E(B-V)_{\text {MW }}$ cut has no effect.

S18 placed a $P_{\text {fit }}>0.001$ cut on the low-redshift sample. While decreasing the fit probability cut to agree with Pantheon gained us $20 \mathrm{SNe}$ Ia, those additional SNe Ia come in a region of parameter space that is poorly modeled by our simulations (see $P_{\text {fit }}$ panel of Figure 7). Additionally, we find that applying a more conservative cut of $P_{\text {fit }}>0.01$ to both the DES and low $z$ subsets resulted in similar statistical constraints on distance. The distribution of low- $z$ sample light-curve parameters after quality cuts is shown in the bottom half of Figure 7.

In the second to last row of Table 1 ("Valid BiasCor"), a few $\mathrm{SNe}$ are lost due to their $\mathrm{SN}$ properties falling within a region of parameter space for which the simulation does not have a bias prediction. Bias corrections are discussed in detail in Section 3.8.1.

Each SN-cosmology analysis that has utilized the historical CfA and CSP-1 low- $z$ samples has dealt with the fact that their Hubble diagram residuals have non-Gaussian tails that are discarded from the cosmological fit. In the last row of Table 1 ("Chauvenets criterion"), we place a final set of cuts before running cosmological parameter fits. This is the same cut on Hubble diagram residuals that was made in S18 of $3.5 \sigma$.

\subsection{Host-galaxy Stellar Masses}

Previous analyses of large SN Ia samples have found a correlation between standardized SN luminosities and hostgalaxy properties (Gallagher et al. 2008; Kelly et al. 2010; Lampeitl et al. 2010; Sullivan et al. 2010, low-z: Childress et al. 2013 and Pan et al. 2014, JLA: B14, PS1: S18). Here we focus on the stellar mass $\left(\mathcal{M}_{\text {stellar }}\right)$ ratio of the host galaxy,

$$
\mathcal{R}_{\text {host }}=\log _{10}\left(\mathcal{M}_{\text {stellar }} / \mathcal{M}_{\odot}\right)
$$

as this quantity has been used in $\mathrm{SN}$-cosmology analyses to correct standardized luminosities since Conley et al. (2011).

Using catalogs from Science Verification DECam images (Bonnett et al. 2016), the directional light radius method (Sullivan et al. 2006; Gupta et al. 2016) is used to associate a host galaxy with each SN Ia. The stellar masses of the DES-SN host galaxies are derived from fitting SEDs to griz broadband fluxes with ZPEG (Le Borgne \& Rocca-Volmerange 2002), where the SEDs are generated with Projet d'Etude des 
Table 2

Simulations Used in DES-SN3YR

\begin{tabular}{|c|c|c|c|c|c|}
\hline & Description & Samples & Scatter Model & Size & Used In \\
\hline & $\mu$ Bias $^{\mathrm{a}}$ & & & & \\
\hline 1 & Fiducial & DES+low $z$ & G10 and $\mathrm{C} 11$ & $\sim 1,300,000 \mathrm{SNe}$ & Sections 3.7 .2 and 3.8 , Figure 7 \\
\hline 2 & Spec. Eff. Syst. & $\mathrm{DES}+$ low $z$ & G10 and $\mathrm{C} 11$ & $\sim 1,300,000 \mathrm{SNe}$ & Sections 3.8 and 4.4, Figure 9 \\
\hline 3 & $\mu$-bias Cosmo. & $\mathrm{DES}+$ low $z$ & G10 and $\mathrm{C} 11$ & $\sim 1,300,000 \mathrm{SNe}$ & Sections 3.8 and 4.5, Figure 10 \\
\hline 4 & $5 \%$ Flux Err. & DES +low $z$ & $\mathrm{G} 10$ and $\mathrm{C} 11$ & $\sim 1,300,000 \mathrm{SNe}$ & Sections 3.8 and 4.8, Figure 10 \\
\hline 5 & $c, x_{1}$ Parent & $\mathrm{DES}+$ low $z$ & $\mathrm{G} 10$ and $\mathrm{C} 11$ & $\sim 1,300,000 \mathrm{SNe}$ & Sections $3.7 .2,3.8$ and 4.3 , Figure 10 \\
\hline 6 & $\begin{array}{l}\text { Two } \sigma_{\text {int }} \\
\text { Validation }^{\mathrm{b}}\end{array}$ & DES +low $z$ & $\mathrm{G} 10$ and $\mathrm{C} 11$ & $\sim 1,300,000 \mathrm{SNe}$ & Section 4.2 \\
\hline 7 & Fake Sample ${ }^{c}$ & DES & $\mathrm{N} / \mathrm{A}$ & $100,000 \mathrm{SNe}$ & Section 6.1 \\
\hline 8 & Fake $\mu$ bias & DES & $\mathrm{N} / \mathrm{A}$ & $\sim 700,000 \mathrm{SNe}$ & Section 6.1, Figure 18 \\
\hline 9 & Stat & $\mathrm{DES}+$ low $z$ & G10 & 200xDES-SN3YR & Section 6.2 \\
\hline 10 & Zero-point $^{\mathrm{d}}$ & $\mathrm{DES}+$ low $z$ & G10 & 200xDES-SN3YR & Section 6.2 , Figure 15 \\
\hline 11 & Scatter Model & $\mathrm{DES}+$ low $z$ & G10 and C11 & 100xDES-SN3YR & Section 6.2 \\
\hline
\end{tabular}

Notes.

${ }^{a}$ Simulations used to compute distance bias ( $\mu$-bias) corrections (Section 3.7).

${ }^{\mathrm{b}}$ Simulations used in the validation of the analysis (Section 6).

${ }^{c}$ Intrinsic scatter set to zero. The simulated fluxes are inserted into DECam images as point sources.

${ }^{\mathrm{d}}$ For each band and each sample, a random zero-point offset is chosen from Gaussian PDF with $\sigma=0.02$ mag.

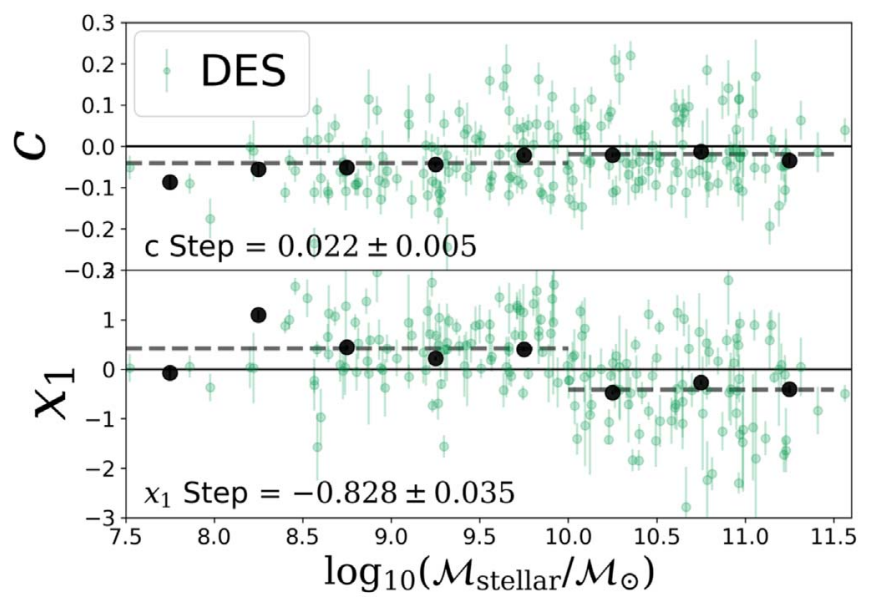

Figure 8. Relations of color, stretch with host-galaxy stellar mass for the DESSN Ia subset before bias corrections have been applied. Steps across $\log _{10}\left(\mathcal{M}_{\text {stellar }} / \mathcal{M}_{\text {sun }}\right)$ are shown in the dashed lines. Binned data points are also shown.

GAlaxies par Synthese Evolutive (PEGASE: Fioc \& RoccaVolmerange 1997).

We define $\delta \mu_{\text {host }}$ to be a distance modulus correction, often referred to as an SN mag-step correction, between $\mathrm{SNe}$ with $\mathcal{R}_{\text {host }}<\mathcal{R}_{\text {step }}$ and SNe with $\mathcal{R}_{\text {host }}>\mathcal{R}_{\text {step }}$ :

$$
\delta \mu_{\text {host }}=\gamma \times\left[1+e^{\left(\mathcal{R}_{\text {host }}-\mathcal{R}_{\text {step }}\right) / 0.01}\right]^{-1}-\frac{\gamma}{2},
$$

where $\mathcal{R}_{\text {step }}=10$. Here, the magnitude of $\delta \mu_{\text {host }}$ is determined by fitting for $\gamma$ where $\delta \mu_{\text {host }}$ is between $[+\gamma / 2,-\gamma / 2]$, with a rapid transition near $\mathcal{R}_{\text {host }}=10$. We find that because we have characterized $\delta \mu_{\text {host }}$ as a step function, its dependence on host mass uncertainties is weak, and therefore uncertainties are not accounted for in this calculation. Additionally, because S18 found little dependence between $\mathcal{R}_{\text {step }}$ and cosmological parameters, we fix the location in our cosmology fit. While
SN Ia host-galaxy properties may change with redshift, we could allow for $\gamma$ to have a redshift dependence, and this possibility is discussed in Section 5.

For galaxies that ZPEG was not able to determine a host mass, we first confirm that the hosts are faint and have not been misidentified, and then we assign them to the low-mass bin. For the DES subset, there are 116 host galaxies with $\mathcal{R}_{\text {host }}<10$ and 91 host galaxies with $\mathcal{R}_{\text {host }}>10$. In Figure 8 we show the distributions of color and stretch as a function of $\mathcal{R}_{\text {host }}$. Correlations between SN Ia light-curve parameters and $\mathcal{R}_{\text {host }}$ have been reported in previous analyses (B14, S18) and are characterized as an average difference (step) for events with $\mathcal{R}_{\text {host }}<10$ and $\mathcal{R}_{\text {host }}>10$. As shown in Figure 8, we find steps in stretch, $\Delta x_{1}=-0.828 \pm 0.035$, and color, $\Delta c=0.022 \pm$ 0.005 . These correlations are significantly larger than what was observed by $\operatorname{S} 18\left(\Delta x_{1}=-0.210 \pm 0.041\right.$ and $\Delta c=0.012 \pm$ $0.004)$. While selection effects may play a role in this difference, a comprehensive study is left to a future work.

\subsection{Simulations}

Here we discuss the use of fakes so that our simulations incorporate the subtleties of the photometric pipeline that cannot be computed from first principles. In addition, we describe here the simulations that are used for determining bias corrections. Because 11 different types of simulations are used throughout the analysis and validation, we refer to Table 2, which lists key attributes for each.

\subsubsection{Fakes Overlaid on Images}

Ideally, a large sample of fakes would be used for characterizing cosmological distance biases. However, our sample of 10,000 fakes that have been processed with SMP is insufficient for multiple reasons. First, 10,000 fakes is more than an order of magnitude smaller than what is needed for the bias-correction sample used in the BBC method. Second, SMP (or other similar methods) is far too computationally intensive for the large number of systematic iterations that are needed to test against varying SN properties and assumptions. These tests 
include multiple iterations of bias corrections, with varying properties, parent populations, and assumptions. For the many analysis iterations that are needed, it is vital to have a rapid method for obtaining simulated catalog photometry that approximates SMP. Using the sample of fakes processed by SMP, we tune our catalog simulations to replicate SMP flux uncertainties. As shown in Figure 2 and Equation (13) of K19, the SN flux uncertainties of the simulated SNe are scaled $\left(\hat{S}_{\text {sim }}\right)$ as a function of host-galaxy surface brightness by the ratio between the observed scatter in the fakes relative to the "observed" scatter in the simulation. As a result, we obtain simulations of DES-SN with the same distribution of photometric uncertainties found in our real data set that can be used for rapid analysis iterations.

\subsubsection{Simulated Light Curves for Bias Corrections}

We use catalog-level simulations of large samples of SNe Ia to model the expected biases in measured distances that follow from the known selection effects and our light-curve analysis. The simulations of the DES-SN and low-redshift samples used for this analysis follow the description of K19. For individual events, distance biases can reach 0.4 mag as shown in Figure 9 of $\mathrm{K} 19$, and it is therefore imperative to have accurate simulations in order to predict biases. The simulation utilizes SNANA and, as detailed in Figure 1 of K19, consists of three main steps: (1) generating a SN source for each epoch (source model), (2) applying instrumental noise (noise model), and (3) simulating DES-SN observing and selection (trigger model). Here we discuss each of these steps briefly, along with specific choices made for this analysis.

Source model: Our simulations first generate rest-frame SN Ia SEDs with the SALT2 model from B14. The model includes SN Ia parent populations of color and stretch, intrinsic luminosity variations, and cosmological effects.

For the DES subset, we test the parent distributions of $c$ and $x_{1}$ found in Table 1 of Scolnic \& Kessler (2016) (hereafter SK16) and find that the high- $z$ row, representative of the populations of all recent high- $z$ surveys combined (SDSS, SNLS, PS1), results in the best agreement in the observed distributions of light-curve parameters when comparing to our DES data set.

For the low- $z$ subset we follow S18. We do not re-derive $x_{1}$ and $c$ parent populations after removal of the CfA1 and CfA2 samples, which compose less than $16 \%$ of the low-redshift sample, because population parameters have little dependence on selection efficiencies.

A model of SN brightness variations, called "intrinsic scatter," is needed to account for the observed Hubble residual scatter that exceeds expectations from measurement uncertainties. Most cosmology-fitting likelihoods characterize the excess Hubble scatter with an additional $\sigma_{\text {int }}$ term added in quadrature to the measured distance uncertainty. From an astrophysical perspective, this $\sigma_{\text {int }}$ term is equivalent to an intrinsic scatter model described by a Gaussian profile where each event undergoes a coherent fluctuation that is $100 \%$ correlated among all phases and wavelengths. Many previous analyses, however, have demonstrated that this simple coherent model does not adequately describe intrinsic scatter. Following K13, we simulate intrinsic scatter with two different intrinsic scatter models in order to investigate the sensitivity to bias corrections and to the $\sigma_{\text {int }}$ approximation in the cosmology-fitting likelihood.
Our intrinsic scatter models include a combination of coherent (Gaussian $\sigma_{\text {int }}$ ) variations and wavelength-dependent SALT2 SED variations that introduce scatter in the generated SN Ia colors. From K13 the first model, "G10," is based on Guy et al. (2010) and describes $\sim 70 \%$ of the excess Hubble scatter from coherent variations, and the remaining scatter from wavelength-dependent variations. The second model, "C11," is based on Chotard et al. (2011) and describes $\sim 30 \%$ of the excess Hubble scatter from coherent variations, and the remaining scatter from wavelength-dependent variations.

Cosmological effects are applied, which include redshifting, dimming, lensing, peculiar velocity, and MW extinction. The simulations used for bias corrections are performed in $\Lambda \mathrm{CDM}\left(w=-1.0, \Omega_{M}=0.3, \Omega_{k}=0.0\right)$. We integrate the redshifted SED with the DECam filter transmission functions to obtain true top-of-atmosphere DECam magnitudes.

Noise model: We simulate the DES-SN cadence and observing conditions (PSF, sky noise, zero-point) using the catalog of DES-SN images. A sample of simulated SNe are drawn from 10,000 random sky locations over the DES-SN observing fields, and for each epoch, the observing conditions are taken from the corresponding DES-SN image. For simulations of more than 10,000 events, sky locations are repeated. We assign a host-galaxy surface brightness and determine photometric uncertainties from PSF, sky, and zeropoint. A photometric uncertainty scaling as a function of $m_{\mathrm{SB}}$ (Sec Section 5 of K19) is then applied. The final product of the noise model is a set of DECam fluxes and flux uncertainties.

Trigger model: The last step is to apply the DES-SN detection criteria and spectroscopic selection. We require two detections on separate nights within 30 days. The spectroscopic selection function for the DES subset $\left(E_{\text {spec }}\right)$ is determined as a function of peak $i$ band magnitude (Section 6.1 of K19).

The low- $z$ subset trigger model, which is detailed in Section 6 of $\mathrm{K} 19$, is based on the procedure developed in B14, S14, and $\mathrm{S} 18$, which assume that the low $-z$ subset is magnitude limited. Separate spectroscopic selection functions are determined for each of the low- $z$ surveys (CfA3, CfA4, and CSP-1). With the assumption of a magnitude limited sample, we are able to obtain good agreement between simulations and data for the distribution of observed redshifts, as shown in Figure 7. However, because it is unclear how selection was done for the low-redshift surveys and that it involved a targeted search of galaxies, we simulate as a systematic uncertainty the assumption that the low $z$ subset is in fact volume limited. The determination of the low- $z$ efficiency function and the implementation of the volume-limited assumption in simulations is discussed in detail in K19.

For a volume-limited low- $z$ subset, redshift evolution of color and stretch are interpreted as astrophysical effects rather than manifestations of Malmquist bias. This allows for the combination of the volume-limited assumption and the uncertainty in parent populations of color and stretch to be analyzed with a single simulation. The parent populations used for the simulations of the low $z$ subset are documented in Table 3.

\subsubsection{Data-simulation Comparisons}

We discuss here the method for evaluating the quality of our simulations. To characterize the level of agreement between data and simulated distributions, we define the $\chi_{\mathrm{p}}^{2}$ between the simulation and data for each population parameter $(p)$ from the 
Table 3

Parent Populations Parameters Table

\begin{tabular}{|c|c|c|c|c|c|c|}
\hline Description & Scatter Model & $c_{\text {peak }}\left(\sigma_{+}, \sigma_{-}\right)$ & $\frac{d c}{d z}$ & $x_{1 \text { peak } 1}\left(\sigma_{+}, \sigma_{-}\right)$ & $x_{1 \text { peak2 }}\left(\sigma_{+}, \sigma_{-}\right)$ & $\frac{a x}{d z}$ \\
\hline DES Systematic & G10 & $-0.065(0.044,0.120)$ & 0 & $0.964(1.232,0.282)$ & $0.000(0.000,0.000)$ & \\
\hline DES Systematic & $\mathrm{C} 11$ & $-0.112(0.003,0.144)$ & 0 & $0.974(1.236,0.283)$ & $0.000(0.000,0.000)$ & \\
\hline Low- $z$ Nominal & G10 & $-0.055(0.023,0.150)$ & -1 & $0.550(1.000,0.450)$ & $-1.500(0.500,0.500)$ & 25 \\
\hline Low-z Vol. Lim. & G10 & $-0.055(0.018,0.150)$ & -1 & $0.200(1.000,0.450)$ & $-2.100(0.500,0.500)$ & 25 \\
\hline
\end{tabular}

Note. Parent population parameters of color $(c)$ and stretch $\left(x_{1}\right)$ used in SNANA simulations for bias corrections. The low- $z x_{1}$ distributions are modeled as two Gaussians with two peaks shown in the table.

comparison of a binned light-curve fit parameter distribution of the data and the normalized binned distribution of the high statistics simulation as follows:

$$
\begin{gathered}
\chi_{\mathrm{p}}^{2}=\sum_{i}\left(N_{i}^{\text {data }}-R \times N_{i}^{\mathrm{sim}}\right)^{2} / N_{i}^{\text {data }}, \\
R=\sum N_{i}^{\text {data }} / \sum N_{i}^{\text {sim }},
\end{gathered}
$$

for parameter bins $i$ and simulation normalization $R$. The simulations have sufficiently high statistics that we ignore statistical fluctuations in the simulations and only use the Poisson uncertainties in the data set.

The agreement between simulations and our DES-SN data set is shown by comparing the distributions of light-curve fit parameters and uncertainties, redshift, and maximum $\mathrm{S} / \mathrm{N}$ among all epochs (SNRMAX) in Figure 7. For each subplot in Figure 7 we report $\left[\chi_{\mathrm{p}}^{2}\right] /[$ dof]. Although only the simulations using the G10 scatter model are shown, the distributions using C11 simulations are indistinguishable by eye.

We find good agreement between the data and simulations for many of the observed parameters, but most notably in redshift (Figure 7). In simulating the DES subset, there was no explicit tuning of the redshift distribution. This gives us confidence in our models used to generate the simulations.

It is important to note that we obtain relatively poor agreement between the DES subset and simulations for the light-curve fit probability $\left(P_{\text {fit }}\right)$ distribution. However, because the agreement for the SNRMAX distribution is good, it is possible that more subtle modeling of photometric uncertainties is needed or that there is variation in the SN population that is not captured by a SALT2 model. Agreement between data and simulations for the low $-z$ subset for SNRMAX and $P_{\text {fit }}$ is worse than for the DES subset. This suggests the need for significant improvements in flux uncertainty modeling. In Section 8.4 we discuss the need for improvements to simulations of $\mathrm{SNe}$ Ia data sets.

\subsection{Cosmology}

Here we discuss the analysis steps taken to extract cosmological distances, fit for nuisance parameters, and correct for expected biases. Additionally, we discuss the production of statistical and systematic distance covariance matrices. Finally, we discuss the cosmological parameter fitting process.

\subsubsection{BEAMS with Bias Corrections (BBC)}

We use the "BBC" fitting method (Kessler \& Scolnic $2017, \mathrm{KS} 17)$ to convert the light-curve fit parameters $\left(m_{B}, x_{1}, c\right)$ into bias-corrected distance modulus values in 20 discrete redshift bins, and to determine nuisance parameters $(\alpha, \beta, \gamma)$. This BBC fit uses a modified version of the Tripp formula (Tripp 1998) where the measured distance modulus $(\mu)$ of each $\mathrm{SN}$ is determined by

$$
\mu=m_{B}-M+\alpha x_{1}-\beta c+\delta \mu_{\text {host }}+\delta \mu_{\text {bias }} .
$$

$\alpha$ and $\beta$ are the correlation coefficients of $x_{1}$ and $c$ with luminosity, respectively, and $M$ is the absolute magnitude of a fiducial SN Ia with $x_{1}=0$ and $c=0$. Following Conley et al. (2011), we include $\delta \mu_{\text {host }}$ (Equation (3)), which depends on $\gamma$. The bias correction, $\delta \mu_{\text {bias }}$, is determined from large simulations (K19) and is computed from a five-dimensional grid of $\left\{z, x_{1}, c, \alpha, \beta\right\}$.

The BBC likelihood $\left(\mathcal{L}_{\mathrm{BBC}}\right)$ is described in detail in Equation (6) of KS17. For the DES-SN3YR sample of spectroscopically classified events, we set the core collapse SN probability to zero and $\mathcal{L}_{\mathrm{BBC}}$ simplifies to

$$
\begin{aligned}
-2 & \ln \left(\mathcal{L}_{\mathrm{BBC}}\right) \equiv \chi_{\mathrm{BBC}}^{2} \\
= & \sum_{i}\left[\left(\mu_{i}-\mu_{\text {model }, i}-\Delta_{\mu, \mathcal{Z}}\right)^{2} / \sigma_{\mu, i}^{2}+2 \ln \left(\sigma_{\mu, i}\right)\right],
\end{aligned}
$$

where the $i$ summation is over SN Ia events, $\mu_{i}$ is the distance modulus of the $i$ th $\mathrm{SN}$ (Equation (5)), $\mu_{\text {model, } i}$ is the distance modulus computed from redshift $z_{i}$ and an arbitrary set of reference cosmology parameters $\left(\Omega_{M}=0.3, \Omega_{\Lambda}=0.7, w=-1\right)$, and $\Delta_{\mu, \mathcal{Z}}$ is the fitted distance offset in redshift bin-index $\mathcal{Z}$ determined from $z_{i}$. To obtain similar distance constraints in each $\mathcal{Z}$ bin, the redshift bin size is proportional to $(1+z)^{n}$ with $n=6$, and we use $20 \mathcal{Z}$ bins.

Dropping the $i$ index in Equation (6), the distance uncertainty of each $\mathrm{SN}$ is

$$
\begin{aligned}
\sigma_{\mu}^{2}= & C_{m_{B}, m_{B}}+\alpha^{2} C_{x_{1}, x_{1}}+\beta^{2} C_{c, c} \\
& +2 \alpha C_{m_{B}, x_{1}}-2 \beta C_{m_{B}, c}-2 \alpha \beta C_{x_{1}, c} \\
& +\sigma_{\text {vpec }}^{2}+\sigma_{z}^{2}+\sigma_{\text {lens }}^{2}+\sigma_{\text {int }}^{2},
\end{aligned}
$$

where $C$ is the fitted covariance matrix from the light-curve fit, $\sigma_{\mathrm{vpec}}$ is from the peculiar velocity correction, $\sigma_{z}$ is from the redshift uncertainty, $\sigma_{\text {lens }}$ is from weak gravitational lensing, and $\sigma_{\mathrm{int}}$ is determined such that the reduced $\chi_{\mathrm{BBC}}^{2}$ is 1 . Prior to 

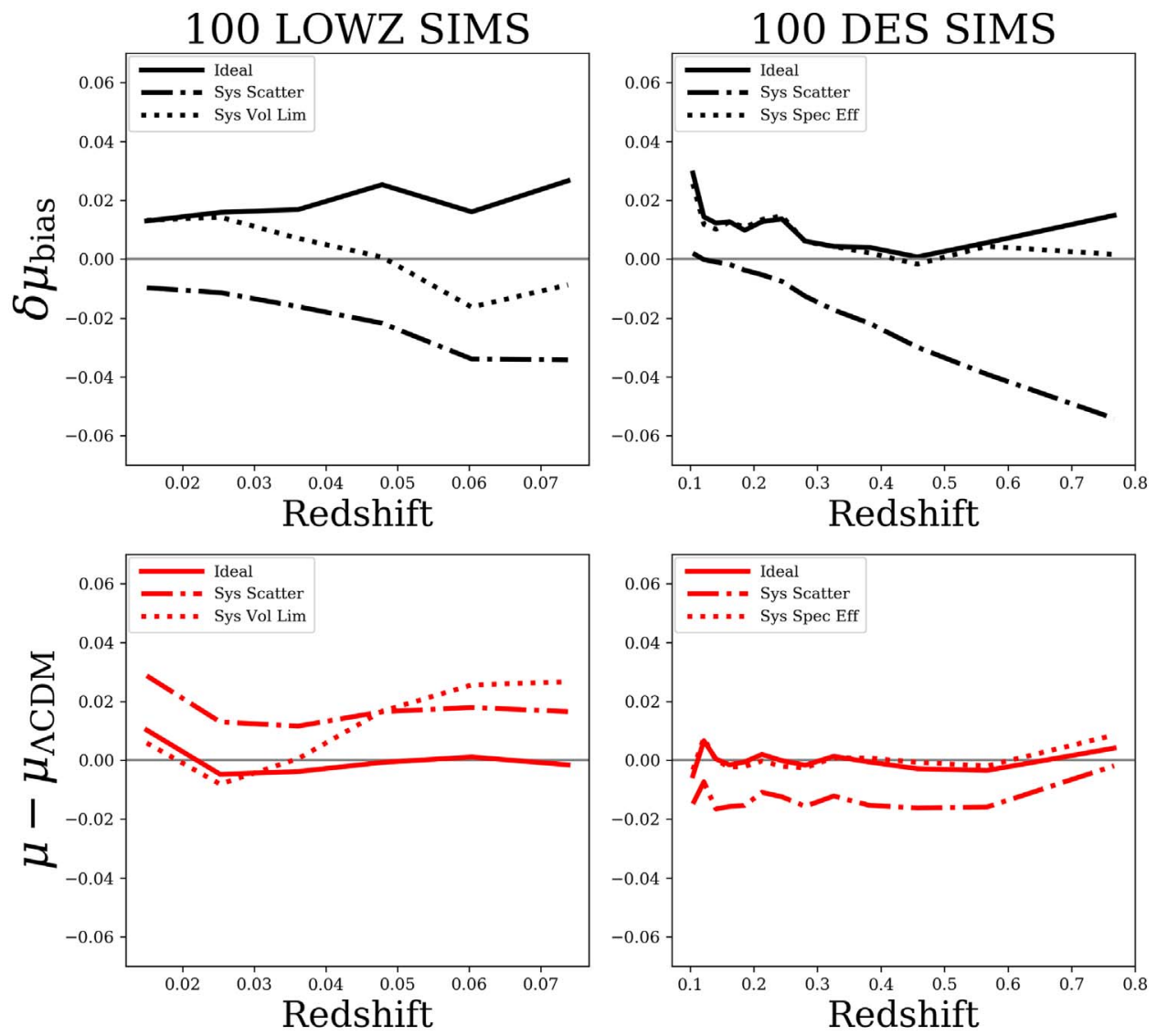

Figure 9. Top: bias correction versus redshift average over 100 DES-SN3YR simulated samples (left: low-z, right: DES-SN). "ideal" corrections have the same scatter model and same selection function in both the simulated data and simulated bias corrections. "Sys scatter" has C11 model for data and G10 model for bias corrections. "Sys Vol Lim" (left) and "Sys Spec Eff" (right) bias corrections are computed using the volume-limited low- $z$ subset and the systematic variation on the spectroscopic efficiency function, respectively (short dashed lines). Bottom: Hubble diagram residuals after bias corrections are applied. Residuals are consistent with zero for the ideal bias corrections.

BBC, $\chi^{2}$-based analyses had ignored the $2 \ln \left(\sigma_{\mu}\right)$ term of Equation (6) because it resulted in large biases (e.g., Appendix B in Conley et al. 2011). However, KS17 found that including the $\delta \mu_{\text {bias }}$ term removes the previously found biases, and that including the $2 \ln \left(\sigma_{\mu}\right)$ is essential within the BBC framework.

To fit for cosmological parameters in Section 3.8.3, the redshift-binned Hubble diagram is defined from the BBC fit as

$$
\begin{gathered}
\langle z\rangle_{\mathcal{Z}}=\operatorname{INVERSE}\left(\left\langle\mu_{\text {model }, i}\right\rangle_{\mathcal{Z}}\right) \\
\langle\mu\rangle_{\mathcal{Z}}=\Delta_{\mu, \mathcal{Z}}+\left\langle\mu_{\text {model }, i}\right\rangle_{\mathcal{Z}}
\end{gathered}
$$

where INVERSE is a numerical function that computes redshift from the distance modulus and $\left\langle\mu_{\text {model, } i}\right\rangle_{\mathcal{Z}}$ is the weighted average $\mu_{\text {model }, i}$,

$$
\left\langle\mu_{\text {model }, i}\right\rangle_{\mathcal{Z}}=\left[\sum_{z_{i} \in \mathcal{Z}} \mu_{\text {model }, i} / \sigma_{\mu, i}^{2}\right] /\left[\sum_{z_{i} \in \mathcal{Z}} \sigma_{\mu, i}^{-2}\right],
$$

where the summations are over the subset of DES-SN3YR events in redshift bin $\mathcal{Z}$.

$\mathcal{L}_{\mathrm{BBC}}$ has three types of approximations. The first is the characterization of intrinsic scatter with a single $\sigma_{\text {int }}$ term in $\mathcal{L}_{\mathrm{BBC}}$, which does not correspond to either of the scatter models. The second approximation in the $\chi^{2}$ likelihood is the implicit assumption of symmetric Gaussian uncertainties on the bias-corrected SALT2 fitted parameters (March et al. 2011).
The final type of approximation is in the modeling for bias corrections, which are determined from simulations that include approximations resulting from limited precision in the: SALT2 model, color and stretch populations, intrinsic scatter model (G10 and C11), estimation of SMP flux uncertainties, and choice of cosmology parameters.

The first two approximations are not included as systematic uncertainties because KS17 performed extensive testing on nearly one million simulated $\mathrm{SNe}$ Ia to demonstrate that the resulting $w$ bias is below 0.01 . In addition, we perform our own DES-SN3YR validation tests for both bias and uncertainty in Section 6. Lastly, the third set of approximations in simulated bias corrections are included as systematic uncertainties.

Here we illustrate the $\mathrm{BBC}$ method using 100 realizations of DES-SN3YR for both the G10 and C11 scatter models. The top panels of Figure 9 show the calculated $\delta \mu_{\text {bias }}$ as a function of redshift. In the bottom panels of Figure 9, we show the BBCfitted distance residuals after bias corrections have been applied. For our "ideal" analysis (solid lines), the bias corrections have the same scatter model and same selection function as the simulated data, and the BBC-fitted distance residuals are consistent with zero. While the average $\mu$-bias correction differs by up to 0.08 mag when the wrong model of intrinsic scatter is used for bias corrections ("Sys Scatter"), the BBC-fitted distance residuals differ by no more than $\sim 0.02$ mag. The reduced effect on distance biases is caused by the different $\beta$ values from the BBC fit. 
Table 4

Sources of Uncertainty

\begin{tabular}{|c|c|c|}
\hline$\overline{\text { Size }^{\mathrm{a}}}$ & "Description & Reference \\
\hline & SN Photometry & \\
\hline $1 \mathrm{mmag}$ & From astrometry & Bernstein et al. (2017) \\
\hline $1 \mathrm{mmag}$ & Nonlinearity of the CCD & Bernstein et al. (2017) \\
\hline $1-2 \mathrm{mmag}$ & Photometric zero pointing & B18-SMP \\
\hline \multirow[t]{2}{*}{3 mmag } & Photometric bias determined by fakes & B18-SMP \\
\hline & Calibration & \\
\hline $6 / \sqrt{3} \mathrm{mmag}$ & DECam $\sigma_{\text {uniformity }}$ & Burke et al. (2018) \\
\hline $0.6 \mathrm{~nm}$ & DECam filter curves uncertainty & Abbott et al. (2018) \\
\hline$[-2,-2,-1,5] \mathrm{mmag}$ & Modeling of C26202 implemented as coherent shift $[g, r, i, z]$ & Figure 4 \\
\hline $5 \mathrm{mmag} / 700 \mathrm{~nm}$ & HST Calspec spectrum modeling uncertainty & Bohlin et al. (2014) \\
\hline $1 / 3$ No SuperCal & SuperCal process & S18, Scolnic et al. (2015) \\
\hline Following S18 & Low- $z$ samples photometric calibration. & S18, CfA3-4, CSP-1 \\
\hline Following S18 & Low- $z$ samples filter curve measurement. & S18, CfA3-4, CSP-1 \\
\hline \multirow[t]{2}{*}{ Following B14 } & SALT2 light-curve model calibration & B14 \\
\hline & Bias Corrections (Astrophysical) & \\
\hline Table 3 & $c, x_{1}$ Parent populations resulting in $\Delta \chi^{2}=2.3$ & Section 4.3 \\
\hline $1 / 2(\mathrm{G} 10-\mathrm{C} 11)$ & Model of intrinsic scatter variations & Section 4.2 \\
\hline Two $\sigma_{\text {int }}$ & Separate fit $\sigma_{\text {int }}$ for each subset & Section 4.2 \\
\hline 0.05 in $w$ & ${ }^{\mathrm{b}}$ Cosmology in which the bias correction sample is simulated & Section 4.5 \\
\hline \multirow[t]{2}{*}{$4 \%$ Scaling } & MW Extinction maps & Section 4.9 , Schlafly \& Finkbeiner (2011) \\
\hline & Bias Corrections (Survey) & \\
\hline $3.5 \sigma \rightarrow 3 \sigma$ outlier cut & ${ }^{\mathrm{b}}$ Low- $z$ Hubble diagram outlier cut & Section 4.7 \\
\hline $1 \sigma_{\text {stat }}$ Fluctuation & Spectroscopic selection function statistical fluctuations & Section 4.4, Figure 9 \\
\hline Low-z Selection & Low- $z$ subset magnitude $\rightarrow$ volume-limited survey & Section 4.3 \\
\hline \multirow[t]{2}{*}{$5 \% \sigma_{\text {phot }}$ Underestimation } & ${ }^{\mathrm{b}}$ Incorrect SN photometric uncertainties & Section 4.8 \\
\hline & Redshifts & \\
\hline $4 \times 10^{-5}$ in $z$ & ${ }^{\mathrm{b}}$ Coherent $z$-shift & Section 4.6, Calcino \& Davis (2017) \\
\hline $0.9 \times \beta_{\text {bias }}$ & Peculiar velocity modeling & Section 4.6, Zhang et al. (2017) \\
\hline
\end{tabular}

Notes.

${ }^{\text {a }}$ Size adopted for each source of systematic uncertainty.

${ }^{\mathrm{b}}$ Sources of systematic uncertainty that have not been included in previous analyses.

In summary, $\chi_{\mathrm{BBC}}^{2}$ (Equation (6)) is minimized to determine 24 parameters: a distance modulus in each of the 20 redshift bins ( 2 of which have no events), 3 nuisance parameters $(\alpha, \beta$, $\gamma)$, and the intrinsic scatter term $\left(\sigma_{\text {int }}\right)$. The ensemble of 20 $\left[\langle z\rangle_{\mathcal{Z}},\langle\mu\rangle_{\mathcal{Z}}\right]$ pairs is the redshift-binned Hubble diagram used to fit for cosmological parameters in Section 3.8.3.

\subsubsection{Covariance Matrix}

Following Conley et al. (2011), we compute a systematic covariance matrix $C_{\text {stat+syst }}$, accounting for both statistical and systematic uncertainties. However instead of a $N \times N$ matrix where $N$ is the number of $\mathrm{SNe}$, here $N$ is the number of redshift bins. $C_{\text {stat }}$ is a diagonal matrix whose $\mathcal{Z}$ th entry is the BBCfitted $\mu$-uncertainty in the $\mathcal{Z}$ th redshift bin. The statistical uncertainties from the binned distance estimates form the diagonal matrix $C_{\text {stat }}$, and $C_{\text {syst }}$ is computed from all the systematic uncertainties summarized in Section 4.

Using BBC-fitted distances, for each source of systematic uncertainty ("SYS") we define distances relative to our nominal analysis ("NOM") as follows:

$$
\Delta\left\langle\mu_{\mathrm{SYS}}\right\rangle_{\mathcal{Z}} \equiv\left\langle\mu_{\mathrm{SYS}}\right\rangle_{\mathcal{Z}}-\left\langle\mu_{\mathrm{NOM}}\right\rangle_{\mathcal{Z}}
$$

for redshift bins $\mathcal{Z}$. For each source of systematic uncertainty ("SYS"), we compute $\left\langle\mu_{\mathrm{SYS}}\right\rangle_{\mathcal{Z}}$ by varying that source and recomputing bias-corrected distances. Groupings of systematic variations are outlined in Table 4, and there are a total of 74 individual systematic uncertainty contributions that are evaluated.

We build our redshift-binned $20 \times 20$ systematic covariance matrix $C_{\text {syst }}$ for all sources $\left(\mathrm{SYS}_{k}\right)$,

$$
C_{\mathcal{Z}_{i} \mathcal{Z}_{j}, \text { syst }}=\sum_{k=1}^{K=74} \frac{\partial \Delta\left\langle\mu_{\mathrm{SYS}}\right\rangle_{\mathcal{Z}_{i}}}{\partial \mathrm{SYS}_{k}} \frac{\partial \Delta\left\langle\mu_{\mathrm{SYS}}\right\rangle_{\mathcal{Z}_{j}}}{\partial \mathrm{SYS}_{k}} \sigma_{k}^{2}
$$

which denotes the covariance between the $\mathcal{Z}_{i}$ th and $\mathcal{Z}_{j}$ th redshift bin summed over the $K$ different sources of systematic uncertainty $(K=74)$ with magnitude $\sigma_{k}$.

The binned covariances and distances are provided in machine-readable format in Appendix C. At the link in Appendix $\mathrm{C}$ there is also an unbinned version where the corrections to individual SNe Ia are computed on a 2D 40-bin interpolation grid to create a covariance matrix for the full $\mathrm{SN}$ data set. 
The covariance matrix used to constrain cosmological models is defined as

$$
C_{\text {stat }+ \text { syst }}=C_{\text {stat }}+C_{\text {syst }}
$$

where $C_{\text {stat }}$ is the diagonal matrix of $\sigma_{\mu}^{2}$ binned in redshift and where the indices $\mathcal{Z}_{i}, \mathcal{Z}_{j}$ have been dropped for convenience.

\subsubsection{Fit for Cosmological Parameters}

Constraining cosmological parameters with SN data using $\chi^{2}$ was first adopted by Riess et al. (1998) and again by Astier et al. (2006). The systematic covariance treatment was improved upon by Conley et al. (2011). Here we follow closely the formalism of S18.

Cosmological parameters are constrained by minimizing a $\chi^{2}$ likelihood,

$$
\begin{aligned}
\chi_{\Delta}^{2} & =\boldsymbol{D}^{T} C_{\text {stat+syst }}^{-1} \boldsymbol{D} \\
D_{\mathcal{Z}} & =\langle\mu\rangle_{\mathcal{Z}}-\left\langle\mu_{\text {model }}\right\rangle_{\mathcal{Z}},
\end{aligned}
$$

where $\boldsymbol{D}$ is the vector of 20 distances binned in redshift with each element defined by $D_{\mathcal{Z}}$. In our case $\left\langle\mu_{\text {model }}\right\rangle_{\mathcal{Z}}=$ $+5 \log \left(d_{L} / 10 \mathrm{pc}\right)$, where for a flat ${ } \mathrm{CDM}$ model

$$
d_{L}(z)=(1+z) c \int_{0}^{z} \frac{d z^{\prime}}{H\left(z^{\prime}\right)},
$$

where for simplicity $z \equiv\langle z\rangle_{\mathcal{Z}}$ (Equation (8)) and with

$$
H\left(z^{\prime}\right)=H_{0} \sqrt{\Omega_{M}\left(1+z^{\prime}\right)^{3}+\Omega_{\Lambda}\left(1+z^{\prime}\right)^{3(1+w)}},
$$

where $d_{L}(z)$ is calculated at each step of the cosmological fitting process, and where flatness is assumed in the fits to determine the systematic error budget.

In our analysis we consider two intrinsic scatter models in simulated bias corrections, G10 and C11 (Section 3.7.2), to span the range of possibilities in current data samples. We assign equal probability to each model and compute $\boldsymbol{D}$ and $C_{\text {stat+syst }}$ twice, once for G10 and once for C11. We average the binned distance estimates and covariance matrices for each of the models for intrinsic scatter as follows:

$$
\begin{aligned}
\boldsymbol{D} & =\frac{\boldsymbol{D}^{\mathrm{G} 10}+\boldsymbol{D}^{\mathrm{C} 11}}{2}, \\
C_{\text {stat+syst }} & =\frac{C_{\mathrm{stat}+\text { syst }}^{\mathrm{G} 10}+C_{\mathrm{stat}+\text { syst }}^{\mathrm{C} 11}}{2},
\end{aligned}
$$

where the superscripts "G10" and "C11" indicate bias corrections assuming that specific model of intrinsic scatter. The covariances, $C_{\mathrm{stat}+\text { syst }}^{\mathrm{G} 10}$ and $C_{\mathrm{stat}+\text { syst }}^{\mathrm{C} 11}$, each include the covariance to the other model of intrinsic scatter with scaling $\sigma_{k}=0.5$ following Equation (12). The average in Equation (17) results in a set of cosmological distances that are roughly half way between that of a G10 only assumption and that of a C11 only assumption, where the systematic uncertainty is half the difference instead of the entire difference. Implicit in this characterization of our distances is that the true intrinsic scatter model lies between that of $\mathrm{G} 10$ and $\mathrm{C} 11$ with $68 \%$ confidence.

The fitting of cosmological parameters is done with CosmoMC (Lewis \& Bridle 2002), which is available online and described in Appendix B. We fit the flat $w \mathrm{CDM}$ model above to our DES-SN3YR data set, and we combine with

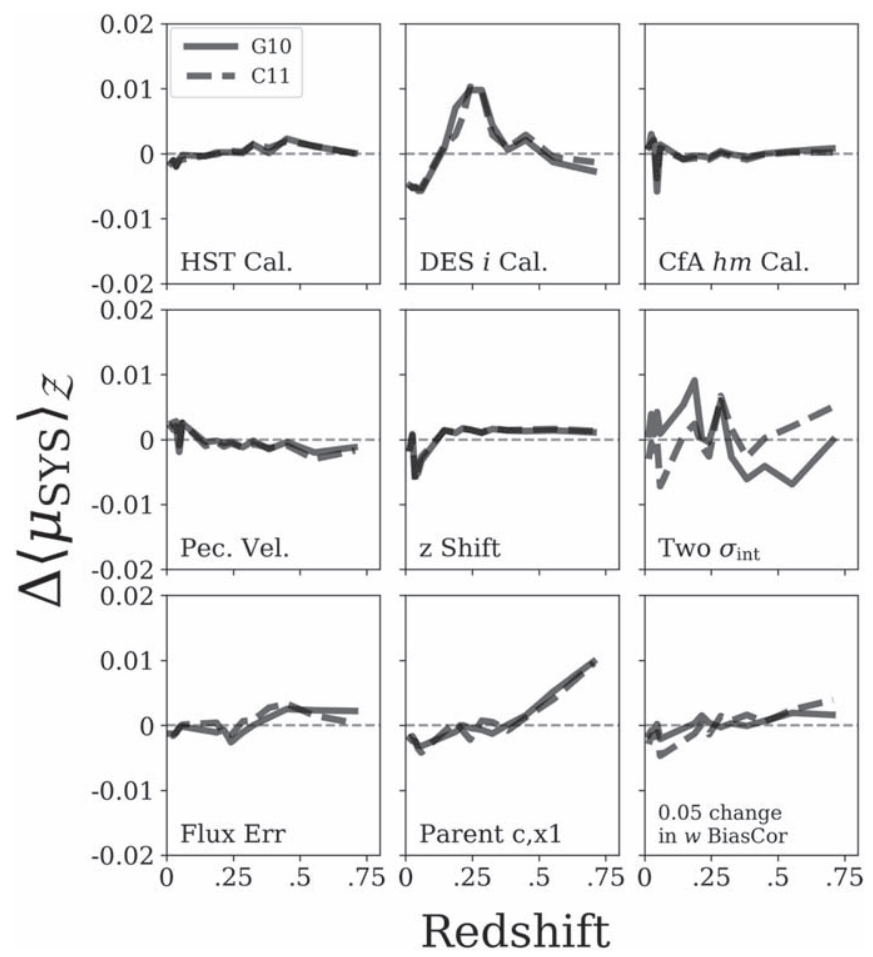

Figure 10. Residuals to the nominal cosmological analysis for the DESSN3YR data set. Distance residuals are calculated for several sources of systematic uncertainty and using bias correction simulations of each model of intrinsic scatter (G10 and C11).

Planck 2016 priors. The best-fit parameters and further extensions to $\Lambda \mathrm{CDM}$ are given in the companion key paper (DES Collaboration et al. 2018). In Section 6 we validate our analysis and uncertainties, and in Section 7 we discuss ongoing development of a more complex likelihood using a Bayesian hierarchical modeling (BHM) framework.

\subsubsection{Blinding the Analysis}

We blind our analysis in two ways simultaneously, as there are a number of steps in the analysis in which one could infer changes to cosmological parameters. First, we blind the binned distances output by the BBC fit. Additionally, to prevent accidental viewing of results, the cosmological parameter constraints were perturbed with unknown offsets.

The cosmological parameters were blinded until preliminary results were presented at the 231st meeting of the American Astronomical Society in January Brout (2018). After unblinding we restored the blinding procedure and made the following changes. First, we fixed the DECam filter transmissions after realizing that atmospheric absorption had been mistakenly ignored. Next, we re-tuned simulations of SMP photometric errors and improved our host-galaxy library. Finally, we included several additional sources of systematic uncertainty: a global shift in our redshifts, two additional calibration systematics (" $1 / 3$ No SuperCal" and "SuperCal Coherent Shift"), and a systematic uncertainty for the use of two $\sigma_{\text {int }}$.

We unblinded again during the internal review process; $w$ increased by 0.024 and the total uncertainty increased by $4 \%$ (0.057 to 0.059$)$. 


\section{Treatment of Systematic Uncertainties}

Here we summarize the treatment and value of each systematic uncertainty from the analysis steps in Section 3 in order to create $C_{\text {sys }}$ from Equation (12). A summary table of the systematics used is provided in Table 4. In Figure 10 we compare the $\Delta\left\langle\mu_{\mathrm{SYS}}\right\rangle_{\mathcal{Z}}$ for several systematics, which allows us to visualize the change in distances for some of the major sources of systematic uncertainty. Systematics that have a large change in distance between low and high redshift (i.e., Parent $c$, $x_{1}$ ) are the largest contributors to the total cosmological parameter error budget, which is discussed in Section 5.

\subsection{Calibration}

There are several systematic uncertainties related to calibration, which include but are not limited to the uncertainty from the photometry (as discussed in B18-SMP), the calibration to the $\mathrm{AB}$ system, and the calibration uniformity across the 10 observing fields. The uncertainty in calibration uniformity across the sky is defined as $\sigma_{\text {syst }}=\sigma_{\text {uniformity }} / \sqrt{N}$, where $N=3$ is the number of DES-SN field groups overlapping PS1 (see C, $\mathrm{S}, \mathrm{X}$ in Section 2), and where we adopt $\sigma_{\text {uniformity }}=6 \mathrm{mmag}$ from Burke et al. (2018). Within a field group (e.g., $\mathrm{C}=\mathrm{C} 1+\mathrm{C} 2+\mathrm{C} 3$ ), we do not count each field (for $N$ ) because the calibration uniformity over $1^{\circ}$ scales is expected to be better than the uniformity over the large separations between field groups.

Uniformity uncertainty due to the location of C26202 is already accounted for here because C26202 is located in one of our SN fields that overlap with PS1. For DES, we combine the photometric uncertainty, uniformity uncertainty, and statistical uncertainty in the $\mathrm{AB}$ calibration and propagate a single uncertainty in the photometric zero-point per band. A final uncertainty is propagated independently by band such that there is a separate entry in $C_{\text {syst }}$ for each band.

To evaluate the agreement of the absolute calibration of the DES-SN fields with the absolute calibration that is used for the low- $z$ sample as described in SuperCal, we utilize the overlap of DES stars with those of PS1 that have also been calibrated following SuperCal. We compute $\chi_{\text {cal }}^{2}$ from the difference in absolute calibration, $\Delta M_{\text {SuperCal }_{i}-\text { DES }_{i} \text {, between PS1-SuperCal }}$ (red) and DES (gray dashed) shown in (Figure 4) as follows:

$$
\chi_{\text {cal }}^{2}=\sum_{i}^{N_{\text {fiter }}} \frac{\left\langle\Delta M_{\text {SuperCal } \left._{i}-\text { DES }_{i}\right\rangle^{2}}\right.}{\sigma_{\text {SuperCal }}^{2}+\sigma_{\text {syst }}^{2}} .
$$

Here $\Delta M_{\text {SuperCal }-\mathrm{DES}_{i}}$ are the offsets to synthetic magnitudes in each filter (red line of Figure 4) relative to the DES calibrated to $\mathrm{C} 26202, \sigma_{\text {SuperCal }}$ is the uncertainty from Scolnic et al. (2015) of $[3,3,2,4]$ mmag in $[g, r, i, z]$ bands, and $\sigma_{\text {syst }}$ is the uncertainty in the uniformity of the fields used for comparison between PS1 and DES $(6 / \sqrt{3} \mathrm{mmag})$. We find that $\chi_{\text {cal }}^{2}=1.5$ for four degrees of freedom, indicating that the DES calibration to $\mathrm{C} 26202$ is consistent with SuperCal.

In order to account for the possibility that the C26202 brightness measured by $H S T$ is biased due to incorrect modeling of the C26202 spectrum, we include a coherent shift in the absolute calibration of DES among all bands simultaneously to SuperCal as an additional uncertainty. That is, we shift our DES-SN magnitudes to an absolute system where the vertical red lines in Figure 4 are defined as zero. Thus, we obtain a new set of SN distances using this new calibration definition, and the $\Delta\left\langle\mu_{\mathrm{SYS}}\right\rangle_{\mathcal{Z}}$ for this choice are propagated in our covariance matrix $C_{\text {syst }}$.

The uncertainty in the calibration of the low- $z$ sample is adopted from SuperCal. Additionally, as was done in S18, we adopt an overall uncertainty associated with the SuperCal itself which Scolnic et al. (2015) characterized as one-third the size of the impact on distances if SuperCal was not applied.

A number of calibration systematics are propagated separately from the absolute and relative calibration treatment provided. Uncertainty in the DECam filter transmission functions propagate to uncertainties in absolute calibration because FGCM utilizes these transmission functions to predict the flux of C26202. A $0.5 \mathrm{~nm}$ wavelength uncertainty arises in the determination of the filter transmission function due to the precision on wavelength in the measurement. Additionally, there is a $0.3 \mathrm{~nm}$ effect arising from illumination lamps on the flat field screen that should be, but are not exactly, on the same optical axis. These two wavelength uncertainties are added in quadrature for a total of $0.6 \mathrm{~nm}$.

We also include the uncertainty in modeling the spectrum of C26202, which is $5 \mathrm{mmag}$ over $700 \mathrm{~nm}$. Lastly, we have not retrained the SALT2 model, and therefore we use the same SALT2 calibration uncertainty as in B14.

We do not include a systematic uncertainty from chromatic corrections, because we have already included FGCM uncertainties that are based on applying these corrections. Furthermore, Lasker et al. (2019) find that if chromatic corrections are not applied, the change in fit $w$ is 0.005 . This change in $w$ is consistent with the statistical uncertainty associated with this correction, and it is well below the systematic uncertainty from our analysis.

\subsection{Intrinsic Scatter Model}

One of the largest systematic uncertainties results from the modeling of intrinsic scatter in the simulations used to predict bias corrections. We include two intrinsic scatter models, G10 and C11, and assign equal probability to each model. Because of the parallel treatment of the scatter models (Equations (17) and (18)), we end up with two sets of nuisance parameters. From here on in this paper, unless otherwise noted, results and nuisance parameters are stated in the context of the G10 model.

As will be shown in Section 5.1.2, the $\sigma_{\text {int }}$ values show $>3 \sigma$ tension when determined separately for the low- $z$ and DES subsets, and this tension persists for both intrinsic scatter models. In addition, our DES-SN $\sigma_{\text {int }}$ value is the smallest of any rolling SN search, suggesting that it is a fluctuation. To account for the possibility that this $\sigma_{\text {int }}$ difference is real, we include a systematic uncertainty based on an analysis using two $\sigma_{\text {int }}$ values, and compare to the nominal analysis that assumes a single $\sigma_{\text {int }}$ value. For the "two $\sigma_{\text {int }}$ " analysis, we scale the spectral flux variations from the intrinsic scatter model (G10 or C11) so that analyzing the simulation results in the same $\sigma_{\text {int }}$ values as for the low- $z$ and DES-SN data subsets. These scaled scatter models are used to generate bias correction simulations, and the BBC fit is modified to constrain the ratio, $\sigma_{\mathrm{int}}(\mathrm{low}-z) /$ $\sigma_{\text {int }}(\mathrm{DES}-\mathrm{SN})$, to match that of the data. To summarize, there are two uncertainties related to the unknown source of intrinsic scatter. The first is the relative contribution of coherent versus wavelength-dependent scatter (G10 versus $\mathrm{C} 11$ ). The second is the overall amplitude difference in scatter between the low- $z$ and DES subsets. 


\subsection{Color and Stretch Parent Populations}

In order to estimate the uncertainty in parent color and stretch distributions, we vary the mean and width of each parent population in the simulation until we achieve $>1 \sigma$ deviations between the observed and simulated distributions. We alter the systematic parent populations of color and stretch in order to increase the $\Delta \chi_{p}^{2}$, as defined in Equation (4), by $\sim 2.3$, following Table 39.2 of Tanabashi et al. (2018). The population parameter values used for the nominal and systematic simulations are shown in Table 3. The dependence between observed populations and the spectroscopic efficiency function is sufficiently weak to justify solving for each independently. The differences in assumed parent populations manifest themselves in different bias corrections to the data set and are visualized in the lower-central panel of Figure 10.

For the uncertainty in the parent populations of color and stretch for the low- $z$ subset, this is encompassed in the volumelimited case. In this case, redshift evolution of color and stretch are interpreted as astrophysical effects rather than manifestations of Malmquist bias (Section 3.7.2). A different set of parent population parameters are determined for the volumelimited case and are shown in Table 3.

\subsection{Spectroscopic Selection}

We generate 200 realizations of the DES subset with only statistical fluctuations. We run our $E_{\text {spec }}$ fitting procedure on each realization and find that biases in recovering the input $E_{\text {spec }}$ are limited to $7 \%\left(E_{\text {fit }} / E_{\text {input }}-1\right)$ across the range $19<i_{\text {peak }}<24$ while $1 \sigma$ statistical fluctuations are up to $25 \%$ at $23 \mathrm{rd}$ mag. Because neither the simulation nor BBC fit take into account the statistical uncertainty in the $E_{\text {spec }}$, we adopt the $1 \sigma$ statistical fluctuation and propagate it as a systematic uncertainty.

We do not include a spectroscopic efficiency systematic for the low- $z$ subset. Instead, the low- $z$ subset is assumed to be magnitude limited and the systematic uncertainty for simulating this sample is to model it as volume limited (see Table 3 and Section 6.2 of K19).

\subsection{Cosmology Assumption in Bias Corrections}

We include the systematic uncertainty from our choice to simulate selection biases with a fixed set of $w C D M$ parameters $\left(\Omega_{M}=0.3, \Omega_{\Lambda}=0.7, w=-1\right)$. Here we redetermine the distance bias after changing the reference cosmological model in our simulations to $w_{\text {ref }}=w_{\text {bestfit }}-0.05$, a change that matches the statistical precision of our measurements. The difference in distance biases for these two reference cosmology values is illustrated in Figure 10 and is less than 2 mmag across the entire redshift range.

\subsection{Redshifts}

We include two systematic uncertainties for our treatment of redshifts. The first is from our modeling of the peculiar velocities, and following Zhang et al. (2017) we modify the light-to-matter bias parameter $\left(\beta_{\text {bias }}\right.$ ) by $10 \%$ and remeasure the redshift corrections. The second is a coherent shift in each redshift of $4 \times 10^{-5}$ as conservatively constrained in Calcino \& Davis (2017).

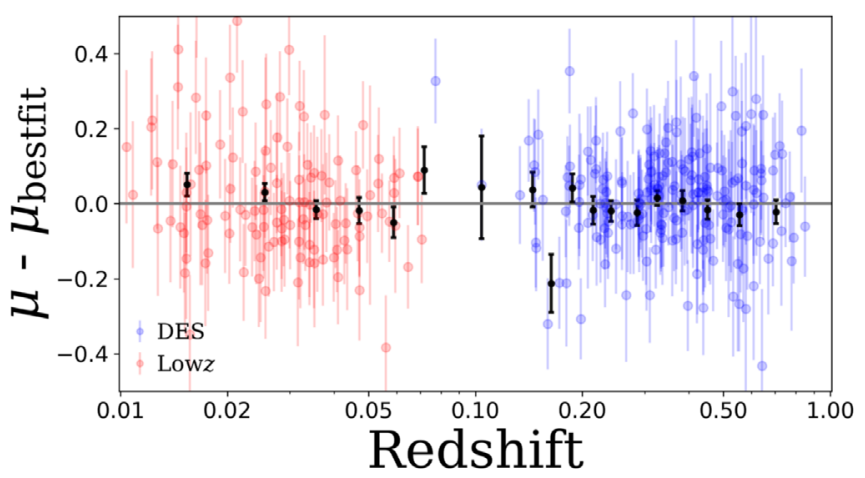

Figure 11. Residuals in distance to the best-fit flat $w C D M$ cosmology as a function of redshift. Blue: DES subset. Red: low $z$ subset. Black: binned distances used for cosmological fits. BBC-fitted distances shown are averaged assuming each model of intrinsic scatter (G10 and C11).

\subsection{Low-z Hubble Residual Outliers}

We include the systematic uncertainty associated with Hubble residual outlier rejection of SNe Ia in the low- $z$ subset. S18 placed a $3.5 \sigma$ cut on their sample. For our data set of 329 SNe Ia, Chauvenets criterion corresponds to a $3 \sigma$ cut. We investigate the systematic effect of applying both $3.5 \sigma$ and $3 \sigma$ cuts on Hubble diagram residuals to the low- $z$ subset. Because this cut depends on the best-fit cosmological model, it is discussed later in Section 5.2.

\subsection{Photometry}

For the SMP pipeline, there is an additional systematic uncertainty beyond the $0.3 \%$ biases mentioned in Section 3.2. Our SMP pipeline performs stellar position fits independently on each night but uses a globally fitted position of the SN across all nights (B18-SMP). Fitting for stellar positions each night independently accounts for the proper motion of the stars, but B18-SMP find this difference in the treatment of the stars and $\mathrm{SNe}$ can cause a $\sim 2$ mmag bias toward brighter fluxes. This small additional systematic uncertainty is added in quadrature to the calibration uncertainty.

We also test for the underestimation of photometric uncertainties. B18-SMP showed that after using fakes to correct the flux uncertainties as a function of host-galaxy local surface brightness, SN flux uncertainties are accurate to within $5 \%$. We therefore consider a systematic underestimation of uncertainties of $5 \%$.

\subsection{MW Extinction}

Lastly, we account for MW extinction using maps from Schlegel et al. (1998), with a scale of 0.86 based on Schlafly et al. (2010), and the MW reddening law from Fitzpatrick (1999). We adopt a global $4 \%$ uncertainty of $E(B-V)_{\mathrm{MW}}$ based on the fact that Schlafly \& Finkbeiner (2011), in a reanalysis of Schlafly et al. (2010), derive smaller values of reddening by $4 \%$, despite using a very similar SDSS footprint.

\section{Results}

We perform a cosmological fit to our redshift-binned and bias-corrected Hubble diagram. The distances obtained in this analysis are shown as binned residuals to the best-fit cosmology in Figure 11 after bias corrections have been 
Table 5

Nuisance Parameters from BBC Fit

\begin{tabular}{|c|c|c|c|c|}
\hline Parameter & Description & G10 & C11 & $\mathrm{AVG}^{\mathrm{a}}$ \\
\hline$\alpha$ & DES-SN3YR & $0.146 \pm 0.009$ & $0.147 \pm 0.009$ & $0.147 \pm 0.009$ \\
\hline$\alpha$ & DES subset & $0.151 \pm 0.012$ & $0.152 \pm 0.012$ & $0.152 \pm 0.012$ \\
\hline$\alpha$ & Low- $z$ subset & $0.145 \pm 0.014$ & $0.144 \pm 0.014$ & $0.145 \pm 0.014$ \\
\hline$\alpha$ & PS1 & $0.167 \pm 0.012$ & $0.167 \pm 0.012$ & $0.167 \pm 0.012$ \\
\hline$\alpha$ & SNLS & $0.139 \pm 0.013$ & $0.139 \pm 0.013$ & $0.139 \pm 0.013$ \\
\hline$\beta$ & DES-SN3YR & $3.03 \pm 0.11$ & $3.58 \pm 0.14$ & $3.30 \pm 0.13$ \\
\hline$\beta$ & DES subset & $3.02 \pm 0.13$ & $3.56 \pm 0.17$ & $3.29 \pm 0.15$ \\
\hline$\beta$ & Low- $z$ subset & $3.06 \pm 0.19$ & $3.61 \pm 0.24$ & $3.34 \pm 0.15$ \\
\hline$\beta$ & PS1 & $3.02 \pm 0.12$ & $3.51 \pm 0.16$ & $3.26 \pm 0.14$ \\
\hline$\beta$ & SNLS & $3.01 \pm 0.14$ & $3.59 \pm 0.17$ & $3.30 \pm 0.16$ \\
\hline$\gamma$ & DES-SN3YR & $0.025 \pm 0.018$ & $0.016 \pm 0.018$ & $0.021 \pm 0.018$ \\
\hline$\gamma$ & DES subset & $0.009 \pm 0.018$ & $0.004 \pm 0.017$ & $0.007 \pm 0.018$ \\
\hline$\gamma$ & Low- $z$ subset & $0.070 \pm 0.038$ & $0.043 \pm 0.038$ & $0.057 \pm 0.038$ \\
\hline$\gamma$ & PS1 & $0.039 \pm 0.016$ & $0.041 \pm 0.016$ & $0.040 \pm 0.016$ \\
\hline$\gamma$ & SNLS & $0.045 \pm 0.020$ & $0.037 \pm 0.020$ & $0.041 \pm 0.020$ \\
\hline${ }^{\mathrm{b}} \sigma_{\text {int }}$ & DES-SN3YR & $0.094 \pm 0.008$ & $0.117 \pm 0.008$ & $0.106 \pm 0.008$ \\
\hline${ }^{\mathrm{b}} \sigma_{\text {int }}$ & DES subset & $0.066 \pm 0.007$ & $0.088 \pm 0.008$ & $0.077 \pm 0.008$ \\
\hline${ }^{\mathrm{b}} \sigma_{\text {int }}$ & Low- $z$ subset & $0.120 \pm 0.015$ & $0.144 \pm 0.015$ & $0.132 \pm 0.015$ \\
\hline$\sigma_{\text {int }}$ & PS1 & 0.08 & 0.10 & 0.09 \\
\hline$\sigma_{\text {int }}$ & SNLS & 0.09 & 0.10 & 0.10 \\
\hline
\end{tabular}

Notes. Nuisance parameters and uncertainties for the DES-SN3YR and the DES and low- $z$ subsets with comparisons to other data sets. The values for PS1 and SNLS are taken from $\mathrm{S} 18$, which does not report uncertainties on $\sigma_{\text {int }}$.

${ }^{a}$ AVG is presented here solely for comparison purposes and is not used in the analysis.

${ }^{\mathrm{b}} \sigma_{\text {int }}$ uncertainty is the rms from 100 simulated realizations of the data set.

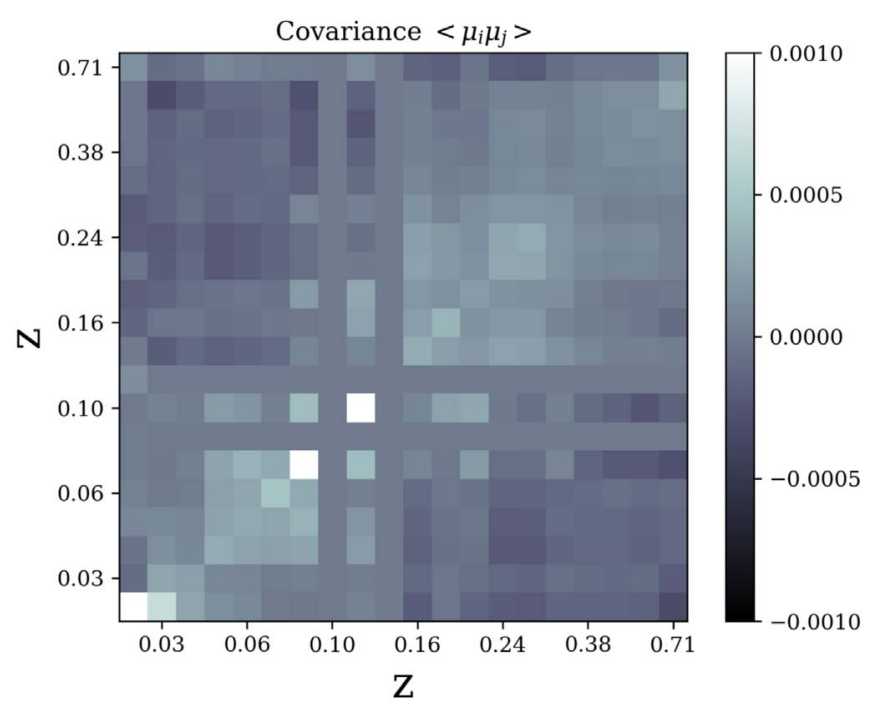

Figure 12. Distance covariance matrix in redshift bins without statistical uncertainties on the diagonal.

applied. The covariance matrix used in our cosmological fits with each of the systematics components $\left(C_{\text {syst }}^{\text {Cosmo }}\right)$ is shown in Figure 12. In this section we report the fit values for the nuisance parameters in Equation (5) and the systematic error budget on cosmological parameters. Several of our results require further discussion, which can be found in Section 8 . We refer the reader to DES Collaboration et al. (2018) for the unblinded best-fit constraints of cosmological parameters.

\subsection{Nuisance Parameters}

The BBC fit output includes four nuisance parameters: $\alpha, \beta$, $\sigma_{\text {int }}$, and $\gamma$. The values for these parameters are summarized in
Table 5, along with a comparison with those of the PS1 and SNLS samples from S18. Here we describe the values found, their consistency with those of previous samples, as well as various perturbations to our analysis and the affect on the recovered nuisance parameters.

$$
\text { 5.1.1. } \alpha, \beta
$$

A comparison of $\alpha$ and $\beta$, the standardization coefficients of stretch and color, with those of the PS1 and SNLS samples, are shown in Table 5. We find that $\alpha$ and $\beta$ are in agreement with various surveys. We test for $\alpha$ or $\beta$ dependence with redshift,

$$
\alpha=\alpha_{0}+z \times \alpha_{1}, \beta=\beta_{0}+z \times \beta_{1},
$$

and we find that $\alpha_{1}$ and $\beta_{1}$ are consistent with zero, with the possible exception of $\beta_{1}$ in our G10 analysis, which we detect at $-1.9 \sigma$. However, in our $\mathrm{C} 11$ analysis we detect $\beta_{1}$ at $-0.5 \sigma$, and thus we consider the evolution in the G10 case to be a statistical fluctuation.

$$
\text { 5.1.2. } \sigma_{\text {int }} \text { and } \sigma_{\text {tot }}
$$

The nominal analysis assumes a single value for the amount of intrinsic scatter needed to bring $\chi^{2} /$ dof $=1\left(\sigma_{\text {int }}\right)$. We perform the nominal analysis twice - once for each model of intrinsic scatter (G10 and C11) - and the values of $\sigma_{\text {int }}$ are found to be 0.094 and 0.117 , respectively (Table 5). These are in agreement with the values found by previous analyses (PS1, SNLS). However, we also examine the $\sigma_{\text {int }}$ for each subset in our analysis. For the DES subset we find $\sigma_{\text {int }}=0.066$ mag for G10 and 0.088mag for C11, which are the smallest observed values of any rolling supernova survey to date using the SALT2 framework. For the low- $z$ subset we find $\sigma_{\text {int }}=0.12$ mag for $\mathrm{G} 10$ and $0.14 \mathrm{mag}$ for C11. In analyzing 100 simulated statistical realizations of DES-SN3YR, we find that 


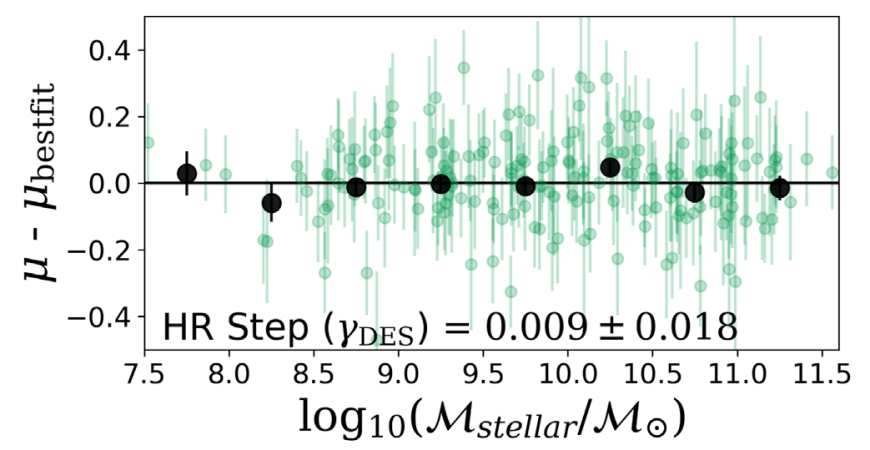

Figure 13. Residuals in distance to the best-fit cosmology as a function of $\log _{10}\left(\mathcal{M}_{\text {stellar }} / \mathcal{M}_{\odot}\right)$ for the DES subset only. Correlation between residuals and mass is characterized as a step function at $10^{10} \mathcal{M}_{\odot}$; however, we find no clear trend in the DES-SN data.

the $\operatorname{rms}\left(\sigma_{\text {int }}\right)$ for the DES subset is 0.007 and for the low- $z$ subset it is 0.015 . Thus, the $\sigma_{\text {int }}$ values for DES-SN and low- $z$ subsets differ by more than $3 \sigma$. In Section 5.2 we discuss the change in fit $w$ if two $\sigma_{\text {int }}$ are used in our analysis $\left(\sigma_{\text {int }}^{\text {low }} z\right.$ and $\sigma_{\text {int }}^{\text {DES }}$ ).

In Table 6 we show the total scatter about the Hubble diagram, $\sigma_{\text {tot }}$, for the subsets in this analysis, and we compare with other surveys. We find the lowest observed value of $\sigma_{\text {tot }}$, 0.129 mag. We also confirm that the 5D bias corrections performed in BBC provide improved Hubble residual scatter over 1D corrections. 1D corrections in this analysis are only used as crosschecks to previous analyses such as B14.

\subsubsection{Host-galaxy Stellar Mass Step $\gamma$}

Somewhat surprisingly, we find little correlation between host-galaxy stellar mass and Hubble diagram residuals $(\gamma=0.025 \pm 0.018)$ for DES-SN3YR. This is driven by the fact that for the DES subset alone, we find no evidence of a correlation $\left(\gamma_{\text {DES }}=0.009 \pm 0.018 \mathrm{mag}\right)$. For the low $z$ subset we find $\gamma_{\text {low- } z}=0.070 \pm 0.038 \mathrm{mag}$, which is consistent with previously seen results. The Hubble diagram residual versus host mass relation for the DES subset are plotted in Figure 13. The DES subset value is $2.4 \sigma$ smaller than $\gamma_{\text {Pantheon }}$ found in S18, which used the same BBC fitting method. As a crosscheck, we have obtained a second set of host-galaxy stellar mass estimates using a different set of SED templates (Bruzual \& Charlot 2003) and fit the griz magnitudes with Le Phare (Arnouts \& Ilbert 2011) spectral fitting code. With the separate set of mass estimates, we find the $\gamma_{\text {DES }}$ value is still consistent with zero (Table 7).

Another crosscheck is to replace the 5D bias correction in the BBC fit with a 1D correction that depends only on redshift, which is similar to the JLA (B14) analysis. We find that using $1 \mathrm{D}$ bias correction in $z$, analogous to that of the JLA (B14) analysis, results in a larger $\gamma_{\mathrm{DES}}$ of $0.041 \pm 0.021 \mathrm{mag}$. This is in agreement with $\mathrm{S} 18$, who find that the 5D bias correction reduces scatter about the Hubble diagram and reduces $\gamma_{\text {DES }}$ by $\sim 0.02$ mag compared to using the 1D bias correction from B14. This will be studied in a forthcoming DES-SN analysis (M. Smith et al. 2018, in preparation) of simulations that include correlations between the $\mathrm{SN}$ properties and the host in simulations. We note that using 5D bias corrections, S18 find significant values for $\gamma$ for each of their subsets of SNe and that the value found here for the low $-z$ sample is in agreement with S18.
Table 6

Comparison of $\sigma_{\text {tot }}$

\begin{tabular}{lll}
\hline \hline Data Set & $\begin{array}{c}\sigma_{\text {tot }}(\mathrm{G} 10) \\
\text { DD [1D] }\end{array}$ & $\begin{array}{c}\sigma_{\text {tot }}(\mathrm{C} 11) \\
5 \mathrm{D}[1 \mathrm{D}]\end{array}$ \\
\hline DES subset & $0.129[0.156]$ & $0.128[0.156]$ \\
Low- $z$ subset & $0.156[0.158]$ & $0.157[0.158]$ \\
DES-SN3YR & $0.142[0.155]$ & $0.141[0.155]$ \\
PS1 & $0.14[0.16]$ & $0.14[\mathrm{~N} / \mathrm{A}]$ \\
SNLS & $0.14[0.18]$ & $0.14[\mathrm{~N} / \mathrm{A}]$ \\
\hline
\end{tabular}

Note. Comparison of rms of Hubble diagram residuals $\left(\sigma_{\text {tot }}\right)$ for the subsets of SNe. Comparisons between performing 5D and 1D bias corrections are also shown. The values for PS1 and SNLS are taken from S18.

Table 7

Systematic Variations for $\gamma_{\mathrm{DES}}$

\begin{tabular}{lrr}
\hline \hline Variation & \multicolumn{1}{c}{$\gamma(\mathrm{mag})$} & \# SNe Ia \\
\hline Nominal & $0.009 \pm 0.018$ & 207 \\
$c>0$ & $0.069 \pm 0.039$ & 70 \\
$c<0$ & $-0.005 \pm 0.020$ & 137 \\
$x_{1}>0$ & $0.018 \pm 0.025$ & 119 \\
$x_{1}<0$ & $-0.013 \pm 0.029$ & 88 \\
no $z$ band & $0.000 \pm 0.021$ & 202 \\
1 D BiasCorr & $0.041 \pm 0.021$ & 207 \\
Diff Img Photometry & $0.001 \pm 0.020$ & 207 \\
$\mathcal{M}_{\text {stellar } \neq \text { null }}$ & $0.010 \pm 0.020$ & 207 \\
$\mathcal{R}_{\text {step }}=10.1$ & $0.021 \pm 0.019$ & 207 \\
$10 z$-bins & $0.015 \pm 0.018$ & 207 \\
Le Phare & $0.008 \pm 0.020$ & 207 \\
\hline
\end{tabular}

Note. Changes in $\gamma$ for the DES subset after perturbations to analysis. Parameter values are shown for the G10 model of intrinsic scatter only.

To examine potential systematics in measuring $\gamma_{\text {DES }}$, Table 7 shows several variations in our BBC fitting procedure. As DECam has better $z$-band sensitivity compared to previous surveys, we ran our analysis without $z$ band and found a consistent $\gamma_{\text {DES }}(0.007 \pm 0.023 \mathrm{mag})$ with a slightly larger uncertainty.

Additionally, because color and stretch are both correlated with host-galaxy stellar mass (Figure 8), we investigate the effect of various cuts to our data set on $\gamma_{\text {DES }}$. Splitting the DES subset into two sub-samples of color, we find that $c>0$ results and $c<0$ differ by $1.6 \sigma$. When performing the analogous test in stretch, we find $x_{1}>0$ and $x_{1}<0$ differ by $1 \sigma$. Statistically these measurements are self-consistent. As a precautionary check, that the small $\gamma_{\mathrm{DES}}$ value is not an artifact of our SMP pipeline; we perform a BBC fit with the DiffImg photometry and find that $\gamma_{\text {DES }}$ remains consistent with zero.

Since we have included host galaxies whose mass could not be determined ( $\mathrm{S} / \mathrm{N}$ too low), and assigned them to the $\mathcal{R}_{\text {host }}<10$ bin, we perform the BBC fit with these events excluded (" $\mathcal{M}_{\text {stellar }} \neq$ null") and still find $\gamma_{\text {DES }}$ consistent with zero. We also test using 10 redshift bins instead of 20 and again the recovered value for $\gamma_{\mathrm{DES}}$ is consistent with zero.

We perform a separate check for redshift evolution of $\gamma$ parametrized as

$$
\gamma=\gamma_{0}+\gamma_{1} \times z
$$

We find $\gamma_{1}$ is consistent with zero for the DES subset $(-0.11 \pm 0.10 \mathrm{mag})$. 
Table 8

$w$ Uncertainty Contributions for $w \mathrm{CDM} \mathrm{Model}^{\mathrm{a}}$

\begin{tabular}{|c|c|c|c|}
\hline Description $^{\mathrm{b}}$ & $\sigma_{w}^{\prime}$ & $\sigma_{w}^{\prime} / \sigma_{w}^{\text {stat }}$ & $w$ shift \\
\hline Total Stat $\left(\sigma_{w}^{\text {stat }}\right)$ & 0.042 & 1.00 & 0.000 \\
\hline Total Syst $^{\mathrm{c}}\left(\sigma_{w}^{\text {total syst }}\right)$ & 0.042 & 1.00 & -0.006 \\
\hline [Photometry and Calibration] & {$[\mathbf{0 . 0 2 1}]$} & {$[\mathbf{0 . 5 0}]$} & {$[-\mathbf{0 . 0 0 5}]$} \\
\hline Low- $z$ & 0.014 & 0.33 & -0.003 \\
\hline DES & 0.010 & 0.33 & 0.001 \\
\hline SALT2 model & 0.009 & 0.21 & -0.003 \\
\hline$H S T$ Calspec & 0.007 & 0.17 & 0.001 \\
\hline $1 / 3$ No SuperCal & 0.005 & 0.12 & -0.001 \\
\hline SuperCal Coherent Shift ${ }^{\mathrm{d}}$ & 0.005 & 0.12 & -0.001 \\
\hline [ $\boldsymbol{\mu}$-Bias Corrections: Survey] & {$[\mathbf{0 . 0 2 3}]$} & {$[\mathbf{0 . 5 5}]$} & {$[-\mathbf{0 . 0 0 1}]$} \\
\hline${ }^{\mathrm{e}}$ Low $-z, 3 \sigma$ Cut & 0.016 & 0.38 & 0.005 \\
\hline Low- $z$ Volume Limited & 0.010 & 0.24 & 0.009 \\
\hline Spectroscopic Efficiency & 0.007 & 0.17 & 0.001 \\
\hline${ }^{\mathrm{e}}$ Flux Err Modeling & 0.001 & 0.02 & -0.001 \\
\hline [ $\boldsymbol{\mu}$-Bias Corrections: Astrophysical] & {$[0.026]$} & {$[0.62]$} & {$[-\mathbf{0 . 0 0 3}]$} \\
\hline Intrinsic Scatter Model & 0.014 & 0.33 & -0.001 \\
\hline$c, x_{1}$ Parent Population & 0.014 & 0.33 & 0.000 \\
\hline${ }^{\mathrm{e}}$ Two $\sigma_{\text {int }}$ & 0.014 & 0.33 & -0.005 \\
\hline MW Extinction & 0.005 & 0.12 & -0.001 \\
\hline${ }^{\mathrm{e}} w, \Omega_{M}$ for bias corr & 0.006 & 0.14 & 0.001 \\
\hline [Redshift $]$ & {$[0.012]$} & {$[0.29]$} & {$[\mathbf{0 . 0 0 3}]$} \\
\hline $\mathrm{e}_{z}+0.00004$ & 0.006 & 0.14 & -0.001 \\
\hline Peculiar Velocity & 0.007 & 0.17 & 0.004 \\
\hline
\end{tabular}

Notes.

a The sample is DES-SN3YR (DES-SN + low- $z$ sample) plus CMB prior.

${ }^{\mathrm{b}}$ Items in [bold] are sub-group uncertainty sums.

${ }^{c}$ The quadrature sum of all systematic uncertainties does not equal 0.042 because of redshift-dependent correlations when using the full covariance matrix.

${ }^{\mathrm{d}}$ Uncertainty is also included in Photometry and Calibration: DES.

${ }^{\mathrm{e}}$ Uncertainty was not included in previous analyses.

Finally, because specific star formation rate (sSFR) is known to correlate with host-galaxy stellar mass (Rigault et al. 2015; Rigault et al. 2018), we explicitly check for a sSFR step with Hubble residuals in the same fashion as Equation (3) and find $0.037 \pm 0.025 \mathrm{mag}$.

\subsection{Systematic Error Budget}

The uncertainties on $w$ are presented in Table 8 for fits to a flat $w$ CDM model with Planck 2016 CMB priors. The systematic uncertainties shown in Table 8 are defined as

$$
\sigma_{w}^{\prime}=\sqrt{\left(\sigma_{w}^{\text {stat }+ \text { syst }}\right)^{2}-\left(\sigma_{w}^{\text {stat }}\right)^{2}}
$$

where $\sigma_{w}^{\text {stat}+ \text { syst }}$ is the uncertainty when only a specific systematic uncertainty (or group of uncertainties) is applied such that $\sigma_{w}^{\prime}$ is the contribution to the total uncertainty from the specific systematic alone. Small shifts in $w$ are expected when including systematic uncertainties due to different inversevariance weights as a function of redshift from the BBC fit. We characterize this effect in Table 8 by including

$$
w-\text { shift }=w_{\text {stat }+ \text { syst }}-w_{\text {stat }},
$$

which is the difference between including and excluding systematic uncertainties. Additionally, we show the contribution to the uncertainty budget for each systematic grouping in column $\sigma_{w}^{\text {syst }}$ and the ratio of systematic uncertainty to statistical uncertainty $\left(\sigma_{w}^{\text {syst }} / \sigma_{w}^{\text {stat }}\right)$. We note that simply summing errors in quadrature from Table 8 will not result in the uncertainty for "ALL" because of redshift-dependent correlations among the systematic effects.

We find that the statistical and systematic uncertainties on $w$ for the DES-SN3YR data set are

$$
\begin{gathered}
\sigma_{w}^{\text {stat }}=0.042, \\
\sigma_{w}^{\text {total syst }}=0.042,
\end{gathered}
$$

where $\sigma_{w}^{\text {total syst }}$ is the $w$ uncertainty from all systematics and excluding statistical uncertainties. This indicates that our result is equally limited by systematic and statistical uncertainties. In Section 8 we discuss how additional data may aid in the reduction of systematic uncertainties.

In Table 8, we break down the independent contributions to the $w$-error budget. We also group the systematic uncertainties into four main categories and find that nearly equal contributions to the total uncertainty from the largest three groupings: (1) photometry and calibration, (2) astrophysical bias corrections, and (3) survey bias corrections, all of which are associated with estimation of distances. The final and smallest grouping (4) describes the systematic uncertainties associated with the redshifts in our analysis.

Photometry and Calibration. Because the low-redshift samples are calibrated to the PS1 absolute magnitude system and because the DES subset has been calibrated to a single CalSpec standard star, we have included an additional calibration uncertainty. We assume coherent offsets to Super$\mathrm{Cal}$ to be our systematic uncertainty for the potential incorrect modeling of the single CalSpec standard. We find that this results in an uncertainty on $w$ of 0.005 . This uncertainty is included in the "DES" calibration uncertainty.

Astrophysical $\mu$-Bias Corrections. As mentioned in Section 4.2, we run the entire analysis pipeline separately for G10 and C11 models of intrinsic scatter. The contribution to the error budget from intrinsic scatter model alone is found to be $\sigma_{w}=0.014$. While we derive separate parent populations associated with each intrinsic scatter model, we also assess the systematic uncertainty in these parent populations. This systematic (" $c, x_{1}$ Parent Pop") is as large as that due to the intrinsic scatter model itself.

Our nominal analysis assumes that all $\mathrm{SNe}$ Ia samples have the same amount of intrinsic variation. However, upon examining the $\sigma_{\text {int }}$ of the DES subset, we find that it is in tension with the value found for the low $z$ subset. We therefore implement another set of BiasCor simulations with separate $\sigma_{\text {int }}$ for each subset and we re-derive distances allowing for two separate $\sigma_{\text {int }}$ in the nuisance parameter fitting stage of SALT2mu. This introduces a systematic uncertainty of 0.014 in $w$.

Survey $\mu$-Bias Corrections. For our nominal analysis, we have followed the treatment in S18 and placed a cut on the Hubble residuals at $3.5 \sigma$ from the best-fit cosmological model. This cut results in a loss of 3 low- $z$ SNe Ia. In addition, we test a $3 \sigma$ cut that results in an additional 2 SNe Ia cut from the low$z$ subset. No SNe Ia from the DES subset are lost to outlier 
cuts. The size of the systematic uncertainty in the outlier cut is $\sigma_{w}=0.016$. The uncertainty arising from statistical fluctuations in the determination of the spectroscopic selection efficiency is 0.007 .

Redshifts. We have included two sources of systematic uncertainty associated with the redshifts used in this cosmological analysis. We find that while both the uncertainty in the peculiar velocities and a systematic redshift measurement offset must be accounted for, their contribution to the $w$-uncertainty budget is not yet comparable to that of distance uncertainties.

New: We have included several sources of systematics that have not been included in previous analyses. These are the redshift uncertainty, an uncertainty on the reported photometric errors, a change in the reference cosmology for simulations, outlier cuts to the low- $z$ subset, and separate $\sigma_{\text {int }} \mathrm{s}$ for each subset. The outlier cut is the largest single source of uncertainty in our analysis, and the separate treatment of $\sigma_{\text {int }}$ is tied for the second largest. When all of these new systematic uncertainties are combined, we find $\sigma_{w}=0.024$, which is comparable to other systematic uncertainty groupings found in in Table 8.

\section{Validation of Analysis}

Here we describe our validation of the analysis using two separate sets of simulations. The first is based on 10,000 fake SNe Ia light curves overlaid on images, and processed with SMP, light-curve fitting, BBC, and CosmoMC. The second test uses a much larger catalog-level simulation from K19, and is processed as if they were a catalog produced by SMP. While these validation tests could have revealed problems leading to additional systematic uncertainties, no such issues were identified, and therefore no additional uncertainties are included. Nonetheless, the validation tests were essential tools in developing the analysis framework, and they provide added confidence in the final analysis. Since these validation tests are not sensitive to errors in calibration, nor to assumptions about $\mathrm{SN}$ properties, we caution their interpretation.

\subsection{Fake SNe Ia Overlaid onto Images}

For the DES subset we simulate a sample of fake SNe Ia light curves and insert light-curve fluxes onto DES-SN images at locations near galaxy centers. B18-SMP use these fake transients to (1) measure biases associated with SMP, (2) assess the accuracy of SMP uncertainties and subsequently adjust errors in both data and simulations, and (3) optimize the photometric pipeline outlier rejection. Here, we take this fake analysis one step further and perform a cosmology analysis resulting in a measurement of $w$. The benefit is that we can investigate potential biases that are not correctly modeled in early stages of the analysis (i.e., the search pipeline), which could propagate to uncorrected biases in distances and fit cosmological parameters. While previous analyses (e.g., Astier et al. 2006, B14) used fake transients to test their photometry pipelines, our test is the first to validate the cosmology analysis with fakes.

A sample of 10,000 fake SNe Ia light curves are discovered by DiffImg, processed by SMP, bias corrected with BBC, and run through our cosmological parameter fits with CosmoMC in the same fashion as the real data set. A detailed description of the analysis of the 6586 fakes that pass quality cuts is found in Appendix D. The agreement between the BiasCor sample used to model the fakes data set, and the fakes processed through our

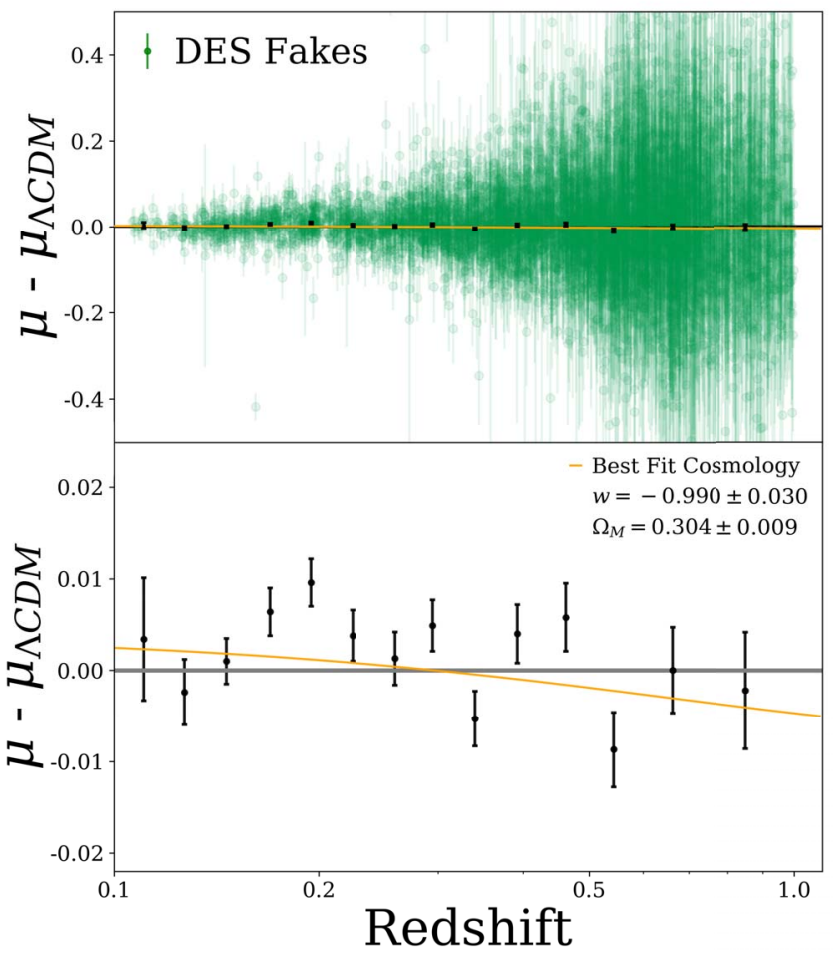

Figure 14. Hubble residuals from 6586 fake $\mathrm{SNe}$ using the same analysis procedure as for the DES-SN3YR sample, except the CMB prior is replaced with a Gaussian $\Omega_{M}$ prior. Upper: zoomed out showing $\mathrm{BBC}$ bins and individual SNe on same $y$-scale as Figure 11. Lower: zoomed in to show BBCbinned residuals more clearly. The black horizontal line corresponds to the flat $\Lambda$ CDM model $\left(\Omega_{M}=0.3\right)$ used to generate the fakes. The orange line is the best-fit $w$ CDM model, and best-fit $w$ and $\Omega_{M}$ are shown on the lower panel.

analysis pipelines are shown in Figure 18, which is analogous to Figure 7 for the real data. We analyze the fakes with $\mathrm{BBC}$ (Section 3.8.1) to produce a redshift-binned Hubble diagram, and the $\mathrm{BBC}$ distances residuals to the input $\Lambda \mathrm{CDM}$ distances are shown in Figure 14 as a function of redshift. Cosmological fits to the fake SNe Ia are not performed with Planck 2016 CMB priors because the underlying cosmology of Planck is unknown and therefore we cannot check for cosmological parameter biases. Instead, we perform $w C D M$ fits on the binned distances with a prior on $\Omega_{M} \sim \mathcal{N}(0.3,0.01)$. The $\chi^{2} /$ dof in Figure 14 is 2.5; however, the amount of additional distance uncertainty per $\mathrm{SN}$ required to bring $\chi^{2} /$ dof to unity is 4 mmag, which is much smaller than the intrinsic scatter in the DES-SN subset $\left(\sigma_{\text {int }}^{\text {DES }}=0.070 \mathrm{mag}\right)$. Finally, we find $w=-0.990 \pm 0.030$ (yellow), which is consistent with the $\Lambda C D M$ cosmology in which the fake SNe Ia were generated. Since the $w$ bias from analyzing the fakes is consistent with zero, we do not assign a systematic uncertainty from this test.

\subsection{Large Catalog-level SNANA Simulations}

In contrast to the analysis with fakes, we perform our analysis on SNANA simulations that include systematic variations in both the DES-SN and low- $z$ samples. These simulations are used to check that our recovered cosmological parameters and their uncertainties are determined accurately.

We begin by generating 200 data samples of comparable size to the DES-SN3YR, each with independent statistical fluctuations, and with no systematic variations. Here we simulate and analyze using the G10 model only. Each sample is processed 
Table 9

Summary of Validation Results from Simulations

\begin{tabular}{lccccc}
\hline \hline Column & 1 & 2 & 3 & 4 & \\
Row & $\bar{w}+1^{\mathrm{a}}$ & $\mathrm{rms}\left(w_{\text {statonly }}\right)$ & $\left\langle\sigma_{w}\right\rangle$ & $R_{\sigma}{ }^{\mathrm{b}}$ & Description \\
\hline 1 & $-0.0029 \pm 0.0035$ & 0.047 & 0.050 & 1.06 & Statistical \\
2 & $-0.0039 \pm 0.0072$ & 0.098 & 0.101 & 1.03 & ZP Systematic $^{\mathrm{c}}$ \\
3 & $-0.0046 \pm 0.0053$ & 0.076 & 0.076 & 1.00 & Intrinsic Scatter Model $^{\mathrm{d}}$ \\
\hline
\end{tabular}

Notes. 200 "DES Like" realizations with and without input sources of systematic uncertainty. All simulations are fit with an $\Omega_{M}=0.3 \pm .01$ prior.

${ }^{\mathrm{a}}$ Inverse-variance weighted average.

${ }^{\mathrm{b}} R_{\sigma}$, defined in Equation (24).

${ }^{c}$ ZP Systematic corresponds to a zero-point magnitude offset drawn from a Gaussian distribution of width 0.02 mag for each band independently in each of the 200 simulations.

${ }^{\mathrm{d}}$ Intrinsic scatter model systematic corresponds to 200 simulations, 100 with each model of intrinsic scatter (G10 and C11).

Table 10

Bias and Uncertainty Precision for $\alpha$ and $\beta$

\begin{tabular}{lcccc}
\hline \hline Model $^{\mathrm{a}}$ & $\alpha$ Bias & $\beta$ Bias & $R_{\sigma}(\alpha)$ & $R_{\sigma}(\beta)$ \\
\hline G10 & $-0.0008 \pm 0.0009$ & $-0.024 \pm 0.012$ & 0.91 & 1.16 \\
C11 & $-0.0003 \pm 0.0008$ & $-0.022 \pm 0.016$ & 1.05 & 0.98 \\
\hline
\end{tabular}

Note.

${ }^{a}$ Intrinsic scatter model used in simulated samples for data and bias corrections.

with light-curve fitting, $\mathrm{BBC}$ and CosmoMC using an $\Omega_{M}$ prior of $\mathcal{N}(0.3,0.01)$. We find an average $w$ bias consistent with zero $(-0.0029 \pm 0.0035)$, as shown in row 1 column $1(\mathrm{r} 1, \mathrm{c} 1)$ of Table 9. We also validate the size of our reported uncertainties. We compare the rms of the 200 fitted $w$ values with the average reported $w$ uncertainty, defined as

$$
R_{\sigma}(w)=\left\langle\sigma_{w}\right\rangle / \mathrm{rms}\left(w_{\text {statonly }}\right) .
$$

We find that $R_{\sigma}(w)=1.06$, as shown in $(\mathrm{r} 1, \mathrm{c} 3)$ of Table 9 , indicating that the average reported errors are in agreement with the rms of fitted $w$ values $\left(R_{\sigma}(w)=1\right.$ for perfect agreement). In the top panel of Figure 15, we combine the cosmological parameter posteriors of each of the $200 \mathrm{BBC}$ fits by adding the $\chi^{2}$ contours in order to achieve an "average" contour for the 200 realizations with size corresponding to the typical statistical uncertainty. We also show the best-fit parameters for each of the 200 statistical realizations, calculated from each of the individual posterior peaks, and find that 135 (68\%) of the 200 best fits lie within the $1 \sigma$ contour.

In order to assess the treatment of multiple independent systematics, we run simulations with systematic biases in the zero-point. For each band in each of 200 simulated G10 samples, we perturb the calibration with a randomly selected zero-point shift from a Gaussian distribution with $\sigma=0.02$. This perturbation is for each sample, not each event, and is artificially inflated compared to our data calibration uncertainties ( $\sim 6 \mathrm{mmag})$ in an effort to improve the sensitivity of this test. Upon analyzing these simulations with $\mathrm{BBC}+\mathrm{CosmoMC}$ in which we only account for the zero-point uncertainty in the covariance matrix, we find again that the $w$ bias is consistent with zero $(-0.0039 \pm 0.0072)$, as shown in (row 2, column 1) of Table 9.

In order to demonstrate the effect of the simulations with zeropoint systematics (bottom of Figure 15), we show best-fit parameters from stat-only analyses of each of the 200 simulations with perturbed calibrations (red points). Here we show the average CosmoMC contour using the stat + syst covariance matrix that accounts for zero-point systematic uncertainty (black contour). We find that 139 (70\%) of the 200 best fits (stat-only) fall within the averaged one sigma contour (stat + syst), consistent with a $1 \sigma$ interpretation of the contour. This is also shown in (row 2, column 4) of Table 9, where we demonstrate that after combining with the $\Omega_{M}$-prior, the rms in fit $w$ from analyses with $C=C_{\text {stat }}$ agrees with the average output uncertainties on $w$ from analyses where $C=C_{\text {stat }}+C_{\text {syst }}: R_{\sigma}(w)=1.03$.

In order to validate the treatment of the intrinsic scatter model systematic, we generate 100 realizations of DES-SN3YR using both $\mathrm{G} 10$ and $\mathrm{C} 11$. When analyzing all 200 results from the 100 G10 simulations and $100 \mathrm{C} 11$ simulations together using the averaged distances and covariances of Equations (17) and (18), we find no biases $(-0.0046 \pm 0.0053)$ in recovered cosmological parameters, as shown in $(\mathrm{r} 3, \mathrm{c} 1)$ of Table 9 . We perform the same test on the output uncertainties described previously for the scatter model systematic, and we find $R_{\sigma}=1.00$ (shown in r3, c3 of Table 9). However, because our set of distances used to compute cosmological parameters is averaged between the bestfit distances of each scatter model, we expect subtle biases when evaluating simulations created with a single model of intrinsic scatter. In analyzing only the 100 G10 realizations combined with $\Omega_{M} \sim \mathcal{N}(0.3,0.01)$, we find a $w$ bias of -0.03 , and for the $100 \mathrm{C} 11$ realizations a $w$ bias of +0.03 . We note that combining $\mathrm{SNe}$ with the prior on $\Omega_{M}$ is weaker than combining SNe and Planck Collaboration (2016) CMB constraints by roughly 50\%. The $w$ shift for each scatter model when combining with CMB becomes \pm 0.014 , which is in agreement with the systematic uncertainty derived from Equation (12) and shown in Table 8.

We check the recovery of the BBC-fitted parameters for $\alpha$ and $\beta$ in Table 10. Analogous to $R_{\sigma}(w)$ for $w$-uncertainties (Equation (24)), we define $R_{\sigma}(\alpha)$ and $R_{\sigma}(\beta)$ for statistical-only fits of $\alpha$ and $\beta$, respectively. For both intrinsic scatter models, the $\alpha$ bias is consistent with zero. For $\beta$, there is a hint of bias at the sub-percent level. The uncertainties and $\mathrm{rms}\left(R_{\sigma}(\alpha), R_{\sigma}(\beta)\right)$ agree at the $10 \%$ level for G10 and at the few percent level for C11.

Finally, we generate large simulations of DES-SN3YR with two separate values of $\sigma_{\text {int }}$ for each subset to examine the biases in our analysis. We analyze with BiasCor simulations generated with two separate values of $\sigma_{\text {int }}$ and find that $\langle w\rangle=-1.002 \pm 0.008$ after combining with $\Omega_{M} \sim \mathcal{N}(0.3,0.01)$. The lack of bias when accounting for the two $\sigma_{\text {int }}$ in BiasCor simulations ensures that our treatment of this systematic has been implemented correctly. We also analyze the same realizations our Nominal BiasCor, which use a single value for $\sigma_{\text {int }}$, and find $\langle w\rangle=-1.036 \pm 0.008$. The 

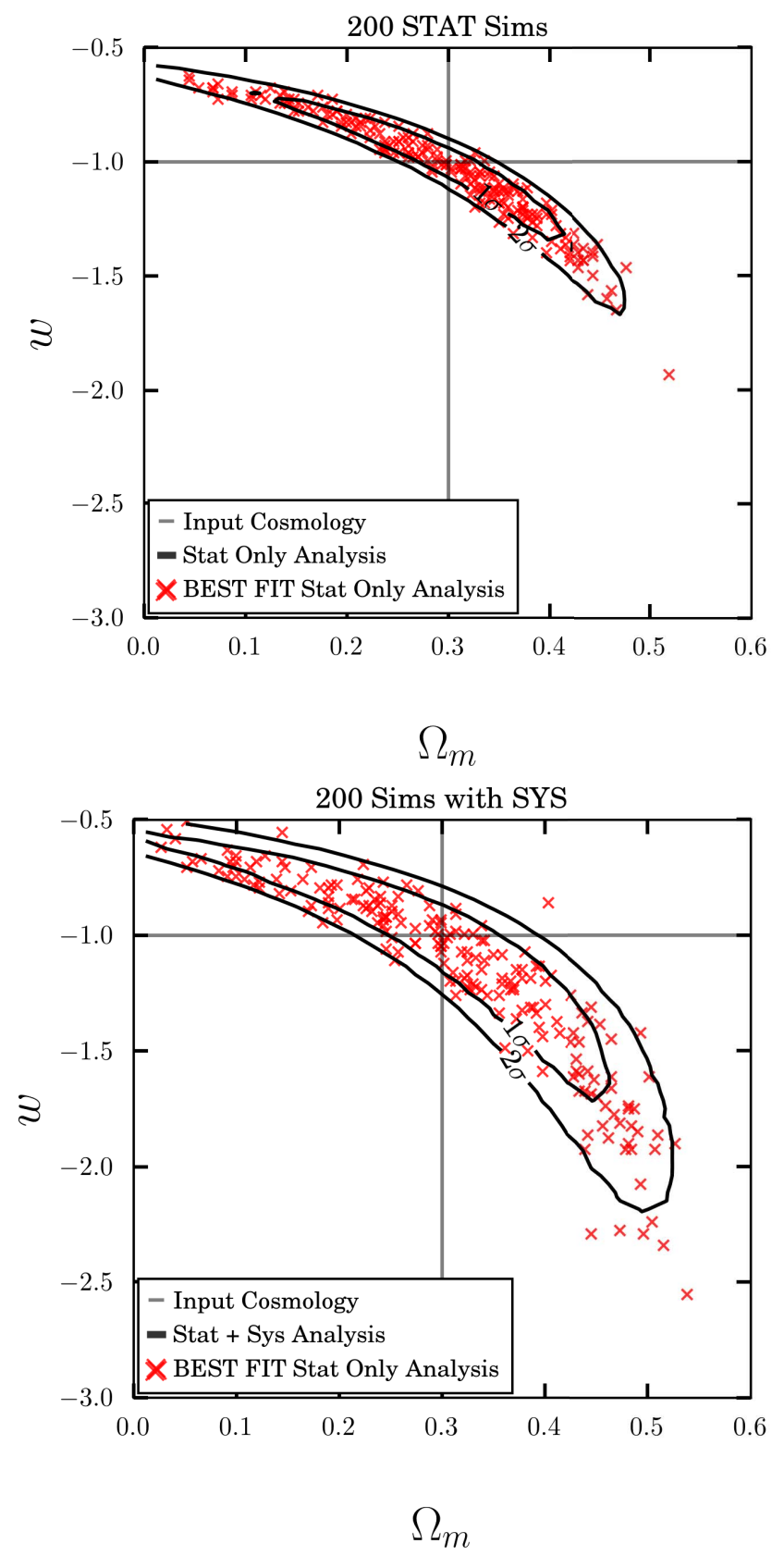

Figure 15. Top: 200 simulated DES-SN3YR data sets with statistical-only fluctuations. Best fits (red) and average posterior (black curve) are shown. Bottom: 200 simulated DES-SN3YR data sets with input calibration systematic of $0.02 \mathrm{mag}$ per filter. The best-fit cosmological parameters for each of the 200 simulations from a $\mathrm{BBC}+\mathrm{CosmoMC}$ analysis using $\left(C=C_{\text {stat }}\right)$ are shown in red. The average posterior from fits to the 200 simulations using $C=C_{\text {stat }}+C_{\text {cal }}$ is shown in black. All simulations are generated in the same input cosmology shown in the gray cross-hairs. All fits have a tophat prior on $\Omega_{M} \in[0,1]$.

observed bias in $w$ when analyzing with our nominal analysis justifies the inclusion an additional systematic uncertainty. We note again that combining SNe with the prior on $\Omega_{M}$ is weaker than combining with $\mathrm{CMB}$ by roughly $50 \%$, and thus the associated systematic uncertainty reported in Table 8 is smaller.

\section{Development of Bayesian Model Fitting}

One of the predominant issues in supernova cosmology is that color and stretch uncertainties are assumed to be Gaussian
Table 11

Comparison of Steve and BBC Nuisance Parameters for DES-SN3YR

\begin{tabular}{lrcc}
\hline \hline & Steve & BBC(G10) & BBC(C11) \\
\hline$\alpha$ & $0.166_{-0.015}^{+0.008}$ & $0.146+ \pm 0.009$ & $0.147 \pm 0.009$ \\
$\beta$ & $3.54_{-0.20}^{+0.12}$ & $3.03 \pm 0.11$ & $3.58 \pm 0.14$ \\
$\gamma$ & $0.029_{-0.028}^{+0.020}$ & $0.025 \pm 0.018$ & $0.016 \pm 0.018$ \\
$\sigma_{\text {int }}($ low $-z)$ & $0.197_{-0.017}^{+0.018}$ & $0.120 \pm 0.015$ & $0.144 \pm 0.015$ \\
$\sigma_{\text {int }}($ DES-SN $)$ & $0.034_{-0.016}^{+0.030}$ & $0.066 \pm 0.006$ & $0.087 \pm 0.006$ \\
\hline
\end{tabular}

Note. $\mathrm{BBC}(\mathrm{G} 10)$ and $\mathrm{BBC}(\mathrm{C} 11)$ refer to intrinsic scatter model used to compute bias corrections.

and symmetric. This assumption is not valid when the uncertainties are comparable to the intrinsic width of the underlying population.

This issue has historically been addressed in two different ways. The first method, used by $\mathrm{BBC}$, determines the true populations of stretch and color (SK16) and in a separate step determines bias corrections with simulations. The second method is to construct a model in which the true underlying values for color and stretch are parametrized (March et al. 2011). BHM have been developed that both utilize bias-correct observables (Shariff et al. 2016) and incorporate selection effects directly into the model (Rubin et al. 2015). Here we summarize a new method called Steve (H18: Hinton et al. 2018), which makes use of detailed SNANA simulations to describe the selection efficiency as part of the likelihood. In addition, Steve does not make assumptions about the underlying intrinsic scatter model, and it uses a parameterized treatment of systematic uncertainties. Although this method is still under development, here we illustrate progress by describing its performance on simulated validation samples and the DES-SN3 YR sample.

H18 validate Steve on the same set of 200 DES-SN3YR simulations as described in Section 6. For the sample generated with the G10 model there is no $w$ bias, while for the sample generated with $\mathrm{C} 11$ there is a bias of 0.05 . When evaluating all 200 validation simulations (G10 and C11 combined), Steve results in an average $w$ bias of +0.03 , and an average $w$ difference $(\Delta w)$ between Steve and the nominal method (BBC + CosmoMC) is +0.04 . The corresponding rms on $\Delta w$ is 0.06 , where this additional scatter comes from the inclusion of fitted parameters in Steve that are fixed in the BBC fit. For example, Steve allows for redshift-dependent populations that are not in the BBC fit because we find no evidence for such a dependence (Section 5.1.1). The extra parameters also result in a larger $w$ uncertainty for Steve in comparison to BBC.

To predict $\Delta w$ for the DES-SN3YR sample, we take the mean $\Delta w$ from the validation sims. For the rms, however, the validation sims are fit with a Gaussian $\Omega_{M}$ prior, $\mathcal{N}(0.3,0.01)$, which is less stringent than the CMB prior used to fit the data. Fitting with both priors shows that the validation uncertainties are over-estimated by a factor of 1.7 , and therefore for DESSN3YR we expect $\operatorname{rms}(\Delta w)=0.04$. On the DES-SN3YR data set, we find a $w$-difference of 0.07 , which is consistent with our simulated prediction of $0.04 \pm 0.04$.

The fitted nuisance parameters from Steve are compared to those from the BBC method in Table 11. The $\alpha_{\text {Steve }}$ value is about 0.02 higher than $\alpha_{\mathrm{BBC}}$, and $\beta_{\text {Steve }}$ is consistent with $\beta_{\mathrm{BBC}}$ using the $\mathrm{C} 11$ intrinsic scatter model in the bias-correction simulation. $\gamma$ for both methods is consistent with zero, although $\gamma_{\text {Steve }}$ is more consistent with $\gamma_{\mathrm{BBC}}$ using the G10 model. Both methods show that the intrinsic scatter term $\left(\sigma_{\text {int }}\right)$ is significantly 


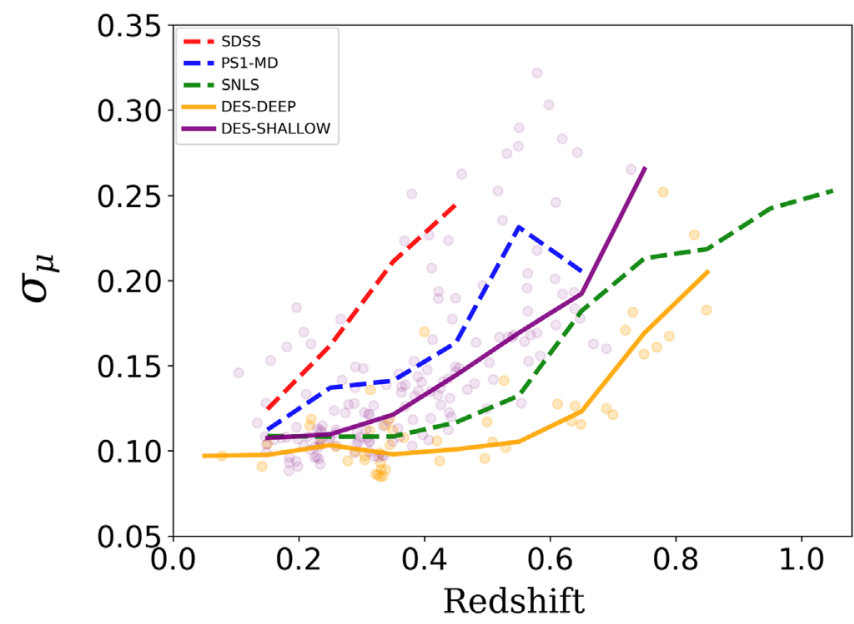

Figure 16. Distance modulus uncertainty vs. redshift for DES, PS1, SNLS, and SDSS. Distance modulus measurement uncertainties reported by each survey are combined with the $\sigma_{\text {int }}$ from this work (DES) and from S18 (PS1, SNLS, SDSS). The colored dots are the individual SNe Ia from the DES shallow (purple) and deep (yellow) fields. The solid (DES) and dashed (other) lines are the binned medians of the respective distributions.

different between the low- $z$ and DES subsets, although the $\sigma_{\text {int }}$ agreement between the two methods is marginal.

\section{Discussion}

\subsection{Comparison with Other Samples}

For the nominal analysis using $\mathrm{BBC}+\mathrm{CosmoMC}$, statistical and systematic uncertainties on $w$ from 329 DES-SN3YR $\mathrm{SNe}$ Ia are 0.042 (stat) and 0.042 (syst). Previous surveys have also found that their statistical and systematic uncertainties are roughly equal. S18 analyzed the PS1 plus low- $z$ subset of the Pantheon sample, and these 451 events result in a statistical and systematic uncertainties on $w$ of 0.046 (stat) and 0.043 (syst). Additionally, in the Joint Light-curve Analysis (B14) they report an uncertainty on $w$ of 0.057 (stat+syst) using 740 SNLS + SDSS+low- $z+H S T$ SNe Ia. The DES-SN3YR result is a notable improvement in constraining power on $w$ for the given sample size (329 SNe Ia), despite the consideration of new sources of systematic uncertainty. Much of this improvement is due to the quality of the DECam CCDs, which include higher sensitivity to redder wavelengths (Holland et al. 2003; Diehl et al. 2008) resulting in improved distance constraints for the most distant supernovae. A comparison of distance uncertainties is shown in Figure 16 using the measurement uncertainties from each respective survey combined with the $\sigma_{\text {int }}$ for each survey that was derived in S18. We find that the DES-SN deep field SNe Ia have smaller uncertainties in distance modulus than SNLS, and the DES-SN shallow field SNe Ia have smaller uncertainties than PS1 but larger than SNLS.

\subsection{Prospects for Improving Systematic Uncertainties}

There are several prospects for future reduction of systematic uncertainties, the largest of which is due to calibration. Multiple improvements are in development for the calibration of the DES photometric system. In this work we used a single HST Calspec standard in one of the $\mathrm{SN}$ fields to link our photometric magnitudes to the $\mathrm{AB}$ system. In the last two seasons of the survey, we measured ugriz $Y$ photometry for two other CalSpec standards (DA White Dwarfs) that are within the
DES footprint. We have identified a large number of hot DA White Dwarfs $(\sim 100)$ that are faint enough to avoid saturation in our nominal $90 \mathrm{sec}$ exposures but bright enough to collect ground-based spectra of suitable quality for analysis. In addition to absolute calibration, there are also prospects to reduce the uncertainty due to internal calibration. A publication dedicated to detailing bandpass measurement corrections, stellar catalog improvements, and code improvements is forthcoming.

The next largest source of systematic uncertainty is from the model of intrinsic scatter, with $\sigma_{w}=0.014$. Our low- $z$ subset is redder than the DES-SN and other high- $z$ populations because it was part of a targeted selection of host galaxies. The different color population of the low- $z$ subset results in increased sensitivity to the change in scatter model. Additionally, we find that our data set is more sensitive to the intrinsic scatter model uncertainty than S18. This is because the low-z sample is a larger fraction of our cosmological sample (DES-SN3YR) in comparison to S18. The two intrinsic scatter models are nearly $8 \mathrm{yr}$ old, and there are currently more than $\sim 1300$ SNe Ia from Pantheon + DES-SN that could potentially test the validity of either G10 or C11. We leave this study for a future analysis.

Equally as large as the intrinsic scatter systematic uncertainty is the $w$ uncertainty in the parent populations of color and stretch. There is room for improvement here in two respects: in our analysis methods and in the external data set used.

First, in Section 4.3 of our analysis, we employed a similar ad-hoc procedure as S18 to characterize the uncertainty in the six parameters describing the parent populations of color and stretch based on estimates from Scolnic \& Kessler (2016). A more rigorous method of accounting for these parameter uncertainties and covariances in the BBC method is needed for future analyses.

Second, there is room for improvement from combining with low- $z$ data sets with selection effects that are less severe and better understood. The Foundation supernova survey (Foley et al. 2018; hereafter F18) has the potential to reduce this uncertainty for the low- $z$ sample. Foundation measures light curves for SNe Ia discovered by other rolling surveys (ASASN, ATLAS, etc.) and as a result obtains a sample with less galaxy-selection bias than the current low- $z$ sample. The Foundation low- $z$ survey on the Pan-STARRS telescope has released 225 low- $z$ SNe Ia in DR1, and they are still collecting data with the goal of obtaining up to 800 griz light curves with high quality calibration. They find that the median color $(c=-0.035)$ and stretch $\left(x_{1}=0.160\right)$ of the Foundation SNe Ia DR1 sample are much closer to that of the high- $z$ surveys (i.e., DES: $c=-0.037, x_{1}=0.115$ ) compared to the medians of the distributions of the current low $z$ sample (i.e., CfA,CSP-1: $c=-0.021, x_{1}=0.048$ ).

The Foundation low- $z$ survey may also provide insight into the distribution of residuals to the Hubble diagram at low redshift. In the DES-SN3YR analysis we find a significant source of systematic uncertainty $\left(\sigma_{w}=0.016\right)$ associated with the outlier cut of the low- $z$ subset due to non-Gaussian tails in residuals to the best-fit cosmological model. Additional statistics will better allow us to characterize the distribution of low- $z$ SNe Ia about the Hubble diagram. The non-Gaussian Hubble residuals could be related to data quality, galaxy-selection effects, unknown astrophysical effects, or poor SN modeling, which is more 


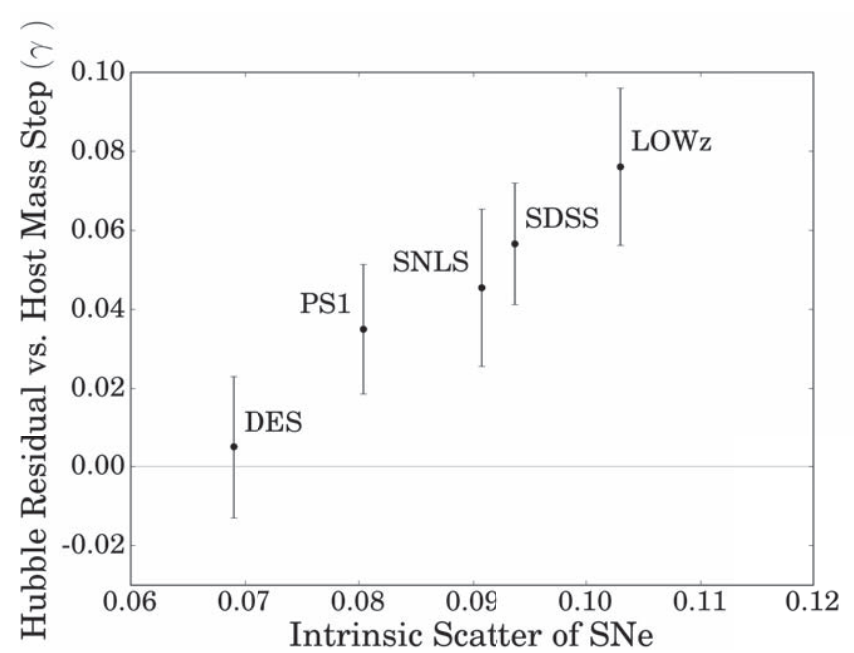

Figure 17. Hubble residual step size in mags $(\gamma)$ as a function of the intrinsic scatter $\left(\sigma_{\text {int }}\right)$ of SNe Ia samples. The largest rolling surveys (DES, PS1, SNLS, SDSS) are shown in addition to the targeted low $z$ subset used in this analysis. Values for the non-DES points are taken from Scolnic et al. (2018) and all are calculated using 5D bias corrections using BBC fit for consistency.

apparent at high $\mathrm{S} / \mathrm{N}$. In any case, the Foundation low- $z$ sample will facilitate further study of this systematic.

\subsection{Host Mass Hubble Residual Step and Intrinsic Scatter}

For DES-SN3YR, we find small values for both $\gamma$ and $\sigma_{\text {int }}$. For the DES subset, $\gamma$ is consistent with zero, indicating no evidence of a correlation between the Hubble residuals to our best-fit cosmology and host-galaxy stellar mass. A significant correlation has been seen to varying degrees in previous analyses (Kelly et al. 2010; Lampeitl et al. 2010; Sullivan et al. 2010; D'Andrea et al. 2011; Gupta et al. 2011; Smith et al. 2012; Rigault et al. 2013; Wolf et al. 2016), and S18 recalculated these quantities within the BBC framework and recovered non-zero steps of size: SDSS $(0.057 \pm 0.015 \mathrm{mag})$, Pan-STARRS $(0.039 \pm 0.016 \mathrm{mag})$, SNLS $(0.045 \pm 0.020 \mathrm{mag})$, and low $-z$ $(0.076 \pm 0.030 \mathrm{mag})$. In an upcoming work, we plan to simulate the correlations between color and host-galaxy stellar mass, and the host-galaxy stellar mass Hubble residual step itself. However, because we recover a non-zero $\gamma$ value for the low- $z$ sample as seen in previous analyses, we suspect that the null correlation found for the DES subset may be the result of selecting a different population of $\mathrm{SNe}$ or host galaxies, but not the result of our analysis techniques.

For future surveys such as LSST and WFIRST, as well as for low-redshift studies of SNe Ia for precision $H_{0}$ measurements, it will be important to improve analysis techniques and study selection effects on the host-galaxy stellar mass correlation, especially if this effect evolves with redshift (Childress et al. 2014). However, in DES-SN3YR we did not find evidence of evolution of $\gamma$ as a function of redshift.

Future SN-cosmology analyses will also be faced with the decision whether to include two $\sigma_{\text {int }}$. We have found that the $\sigma_{\text {int }}$ values of the low- $z$ and DES subsets are incompatible. In this work our nominal analysis assumes a single value for $\sigma_{\text {int }}$ for historical reasons; however, we find that the systematic associated with this choice is one of the largest sources of uncertainty in our analysis. Interestingly, looking at recent SNe Ia data sets all analyzed with the SALT2 model and BBC 5D formalism, we find a correlation between $\gamma$ and $\sigma_{\text {int }}$ of the individual samples. Figure 17 shows this correlation for the DES and low $-z$ subsets, as well as for the other surveys analyzed in S18. The incompatibility between the DES subset and the low $-z$ subset does not appear to be unique to the low$z$ data used in this analysis (CfA and CSP-1). Foley et al. (2018) report in their initial data release an intrinsic scatter of 0.111 . The $\sigma_{\text {int }}-\gamma$ correlation could be a measurement artifact, or $\sigma_{\text {int }}$ could have astrophysical dependence. Future work will be focused on probing the possibility of a redshift-dependent intrinsic scatter term but will require the use of larger data sets. As uncertainty budgets shrink with new and larger SNe Ia samples, it will become important for future analyses to better characterize this effect and model it in simulations.

\subsection{Simulating SNe Ia Samples}

We have shown that there is still room for improvement in modeling the simulated $P_{\text {fit }}$ distributions (Figure 7). We find that the agreement for the DES-SN sample is better than that of the low- $z$ sample, especially in the range $P_{\text {fit }}<0.5$. This is in part due to the extensive care taken to accurately simulate the DES-SN sample as described in Section 5.1 .1 of K19; however, it is unclear if the lesser agreement in the low- $z$ sample could be the result of unmodeled astrophysics. For the DES-SN sample, there is disagreement between the $P_{\text {fit }}$ distributions of the simulations and the data in the highest bin $\left(P_{\text {fit }}>0.95\right)$. We also see a similar disagreement at the high end when comparing the simulated and fake SN distributions (Figure 18). Since the same discrepancy is seen with the fakes, we rule out the possibility that this is entirely due to SN modeling.

The $P_{\text {fit }}$ agreement between simulations and data for the low$z$ sample is poor at both low and high $P_{\text {fit }}$ (Figure 7). This disagreement will hopefully be improved with the Foundation sample, which will facilitate more accurate simulations. In addition, our DES-SN sample has an additional 90,000 fake supernovae on which we can run SMP and improve our modeling of flux uncertainties in the simulation.

\subsection{Improvements to the Validation}

The validations described in Section 6 are the most extensive for a $\mathrm{SN}$ Ia cosmology analysis pipeline to date. Using fakes we have validated from discovery on DECam images to cosmological parameters, and using catalog-level simulations, and we have validated the $w$ bias $(<0.01)$ and treatment of systematic uncertainty. Future work will expand the number of systematics in Table 9. Additionally, because we utilize BBC, which uses an approximate $\chi^{2}$ likelihood assuming symmetric Gaussian uncertainties, we will validate the BBC confidence region for binned distances, and this will eventually lead to comparing the cosmology likelihoods between the $\mathrm{BBC}+\mathrm{CosmoMC}$ and Bayesian (Steve, Section 7) methods. In addition to comparing likelihoods between methods, ideally we would compare our $\mathrm{BBC}+\mathrm{CosmoMC}$ likelihood to a true likelihood, such as from the Neyman construction (Tanabashi et al. 2018). However, such a comparison is computationally challenging.

\section{Conclusion}

We have presented the analysis, cosmological parameter uncertainty budget, and validation of DES-SN3YR sample consisting of of 207 spectroscopically confirmed Type Ia Supernovae $(0.1<z<0.85)$ discovered by DES-SN and an external sample of 122 low-redshift SNe Ia after quality cuts 

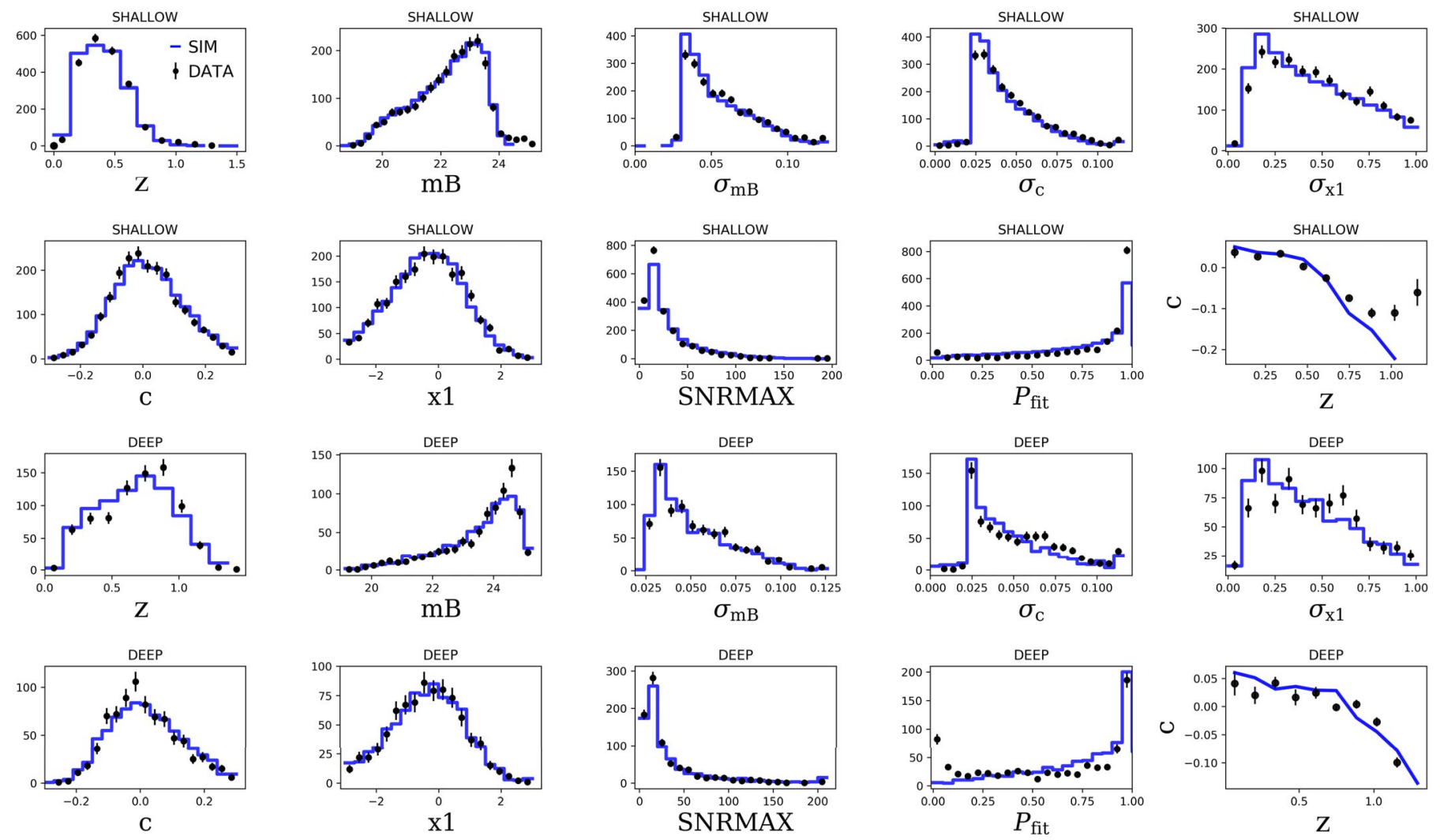

Figure 18. Comparison of 6586 fake supernova light-curve fits with simulations used to compute biases in a fake cosmology analysis.

$(0.01<z<0.1)$. The cosmology constraints are given in the DES-SN key paper (DES Collaboration et al. 2018). We find a total uncertainty $\sigma_{w}=0.057$ (stat+syst). The calibration of the various samples used is the largest source of systematic uncertainty. Additionally we find no correlation between hostgalaxy stellar mass and Hubble residuals to the best-fit cosmology.

Our validation using a population of fake SNe injected onto real images is the first such test for potential biases through the entire SNe Ia discovery, photometry, and analysis pipelines. Resulting biases in distance are limited to $1 \%$, and the fit value of $w$ is consistent with the cosmology in which the fakes were generated. Additionally, we discuss a rigorous method of validating the interpretation of the total uncertainty budget using hundreds of catalog-level simulations. We find that after accounting for sources of systematic uncertainty, there are no significant biases in the cosmological parameter analysis pipeline and that the $\operatorname{rms}(w)$ and the average uncertainty agree to within $6 \%$. The sample from DES used for this analysis is roughly $10 \%$ of the full DES photometric sample, and treatment and validation of systematic $w$ uncertainties will become even more crucial with the larger sample.

This paper has gone through internal review by the DES collaboration. D.B. and M.S. were supported by DOE grant DEFOA-0001358 and NSF grant AST-1517742. This research used resources of the National Energy Research Scientific Computing Center (NERSC), a DOE Office of Science User Facility supported by the Office of Science of the U.S. Department of Energy under contract no. DE-AC02-05CH11231. We are grateful for the support of the University of Chicago Research
Computing Center for assistance with the calculations carried out in this work. Part of this research was conducted by the Australian Research Council Centre of Excellence for All-sky Astrophysics (CAASTRO), through project number CE110001020.

Funding for the DES Projects has been provided by the U.S. Department of Energy, the U.S. National Science Foundation, the Ministry of Science and Education of Spain, the Science and Technology Facilities Council of the United Kingdom, the Higher Education Funding Council for England, the National Center for Supercomputing Applications at the University of Illinois at Urbana-Champaign, the Kavli Institute of Cosmological Physics at the University of Chicago, the Center for Cosmology and Astro-Particle Physics at the Ohio State University, the Mitchell Institute for Fundamental Physics and Astronomy at Texas A\&M University, Financiadora de Estudos e Projetos, Fundação Carlos Chagas Filho de Amparo à Pesquisa do Estado do Rio de Janeiro, Conselho Nacional de Desenvolvimento Científico e Tecnológico and the Ministério da Ciência, Tecnologia e Inovação, the Deutsche Forschungsgemeinschaft, and the Collaborating Institutions in the Dark Energy Survey.

The Collaborating Institutions are Argonne National Laboratory, the University of California at Santa Cruz, the University of Cambridge, Centro de Investigaciones Energéticas, Medioambientales y Tecnológicas-Madrid, the University of Chicago, University College London, the DES-Brazil Consortium, the University of Edinburgh, the Eidgenössische Technische Hochschule (ETH) Zürich, Fermi National Accelerator Laboratory, the University of Illinois at Urbana-Champaign, the Institut de Ciències de l'Espai (IEEC/CSIC), the Institut de Física d'Altes Energies, Lawrence Berkeley National Laboratory, the Ludwig-Maximilians Universität München and the associated 
Excellence Cluster universe, the University of Michigan, the National Optical Astronomy Observatory, the University of Nottingham, the Ohio State University, the University of Pennsylvania, the University of Portsmouth, SLAC National Accelerator Laboratory, Stanford University, the University of Sussex, Texas A\&M University, and the OzDES Membership Consortium.

Based in part on observations at Cerro Tololo InterAmerican Observatory, National Optical Astronomy Observatory, which is operated by the Association of Universities for Research in Astronomy (AURA) under a cooperative agreement with the National Science Foundation.

This paper makes use of observations taken using the AngloAustralian Telescope under programs ATAC A/2013B/12 and NOAO 2013B-0317; the Gemini Observatory under programs NOAO 2013A-0373/GS-2013B-Q-45, NOAO 2015B-0197/ GS-2015B-Q-7, and GS-2015B-Q-8; the Gran Telescopio Canarias under programs GTC77-13B, GTC70-14B, and GTC101-15B; the Keck Observatory under programs U063-2013B, U021-2014B, U048-2015B, U038-2016A; the Magellan Observatory under programs CN2015B-89; the MMT under 2014c-SAO-4, 2015a-SAO-12, 2015c-SAO-21; the South African Large Telescope under programs 2013-1RSA_OTH-023, 2013-2-RSA_OTH-018, 2014-1-RSA_OTH016, 2014-2-SCI-070, 2015-1-SCI-063, and 2015-2-SCI-061; and the Very Large Telescope under programs ESO 093.A0749(A), 094.A-0310(B), 095.A-0316(A), 096.A-0536(A), 095.D-0797(A).

The DES data management system is supported by the National Science Foundation under grant No. AST-1138766 and AST-1536171. The DES participants from Spanish institutions are partially supported by MINECO under grants AYA2015-71825, ESP2015-66861, FPA2015-68048, SEV2016-0588, SEV-2016-0597, and MDM-2015-0509, some of which include ERDF funds from the European Union. IFAE is partially funded by the CERCA program of the Generalitat de Catalunya. Research leading to these results has received funding from the European Research Council under the European Union's Seventh Framework Program (FP7/20072013), including ERC grant agreements 240672, 291329, 306478, and 615929. We acknowledge support from the Australian Research Council Centre of Excellence for All-sky Astrophysics (CAASTRO), through project number CE110001020, and the Brazilian Instituto Nacional de Ciência e Tecnologia (INCT) e-universe (CNPq grant 465376/2014-2).

This manuscript has been authored by Fermi Research Alliance LLC under contract no. DE-AC02-07CH11359 with the U.S. Department of Energy, Office of Science, Office of
High Energy Physics. The U.S. government retains and the publisher, by accepting the article for publication, acknowledges that the U.S. government retains a non-exclusive, paidup, irrevocable, worldwide license to publish or reproduce the published form of this manuscript, or allow others to do so, for U.S. government purposes.

Based in part on data acquired through the Australian Astronomical Observatory, under program A/2013B/012. We acknowledge the traditional owners of the land on which the AAT stands, the Gamilaraay people, and pay our respects to elders past and present.

\section{Appendix A \\ Light-curve Minimization Algorithms}

Light-curve parameter minimization is performed with SNANA's implementation of SALT2 (Guy et al. 2007) based on CERNLIBs MINUIT program (James \& Roos 1975) using MINOS minimization. There is an alternative minimization method, MIGRAD; however, we found that it causes pathological errors for $2 \%$ of our sample of SNe Ia, resulting in incorrect weighting in the SALT2mu distance fitting process. MINOS was found to avoid the pathological color errors, although it is $2.5 \times$ slower than MIGRAD. MIGRAD's speed is useful for development and debugging; however, for the final cosmological analysis we use MINOS.

There are additional fitting anomalies that occur for highSNR events for both MIGRAD and MINOS. These algorithms sometimes fall in false minima, and to avoid these anomalies we add $3 \%$ of peak SN flux to all flux uncertainties on the first of three fit iterations.

\section{Appendix B \\ Public Products Used in the Analysis}

PEGASE (Fioc \& Rocca-Volmerange 1997), Le Phare (Arnouts \& Ilbert 2011), SMP (Brout et al. 2018-SMP), AutoScan (Goldstein et al. 2015), SALT2 models (Guy et al. 2010, B14), SNANA (Kessler et al. 2009b, K19), CosmoMC (Lewis \& Bridle 2002), SNID (Blondin \& Tonry 2007), MARZ (Hinton et al. 2016), ZPEG (Le Borgne \& Rocca-Volmerange 2002), Superfit (Howell et al. 2005).

\section{Appendix C Data Release Products}

DES-SN3YR binned and unbinned distances, measurement uncertainties, and covariance are included at https://des.ncsa. illinois.edu/releases/sn, as well as the full Table 12 in machine-readable format.

Table 12

Light-curve Fit Parameters

\begin{tabular}{|c|c|c|c|c|c|c|c|}
\hline SN-ID & $z_{\mathrm{CMB}}$ & $c$ & $x_{1}$ & $m_{B}$ & $\log \left(\mathcal{M}_{\text {stellar }} / \mathcal{M}_{\odot}\right)$ & $\mu$ & $\mu_{\text {corr }}$ \\
\hline 1248677 & 0.350 & $-0.093 \pm 0.022$ & $1.01 \pm 0.11$ & $21.530 \pm 0.027$ & $9.845 \pm 0.014$ & $41.305 \pm 0.103$ & $0.014 \pm 0.004$ \\
\hline 1250017 & 0.182 & $-0.096 \pm 0.026$ & $1.06 \pm 0.16$ & $20.038 \pm 0.035$ & $8.797 \pm 0.038$ & $39.827 \pm 0.108$ & $0.020 \pm 0.006$ \\
\hline 1253039 & 0.454 & $-0.094 \pm 0.026$ & $0.29 \pm 0.26$ & $22.288 \pm 0.030$ & $10.795 \pm 0.140$ & $41.986 \pm 0.119$ & $-0.018 \pm 0.005$ \\
\hline 1253101 & 0.460 & $0.027 \pm 0.033$ & $1.34 \pm 0.36$ & $22.412 \pm 0.040$ & $8.526 \pm 0.194$ & $41.873 \pm 0.123$ & $-0.001 \pm 0.008$ \\
\hline 1253920 & 0.196 & $-0.085 \pm 0.027$ & $-0.78 \pm 0.14$ & $20.330 \pm 0.033$ & $9.234 \pm 0.033$ & $39.818 \pm 0.110$ & $0.007 \pm 0.007$ \\
\hline
\end{tabular}

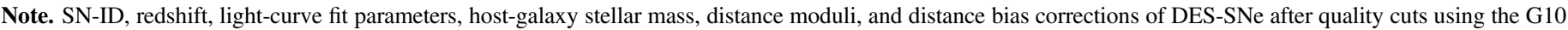

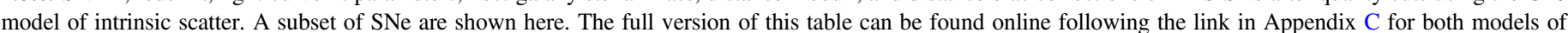
intrinsic scatter (G10 and C11), as well additional information including R.A., decl., fit parameter covariances, 5D bias corrections, and more.

(This table is available in its entirety in machine-readable form.) 


\section{Appendix D Analysis of the Fakes}

Here we describe a few details about the cosmology analysis with fake SN light-curve fluxes overlaid on DECam images. To avoid confusion between two sets of SNANA simulations, we define SIM1 for simulated fluxes overlaid on images and SIM2 for the bias-correction simulation used in the BBC fitting stage.

For SIM1, SN Ia light-curve fluxes were generated in a LCDM cosmology over a redshift range from 0.1 to 1.2. These fluxes were inserted as point sources onto DECam images at galaxy locations chosen randomly with probability proportional to its surface brightness density. The generation of fake light curves and the procedure for image overlays are described in detail in Section 2 of K19. DiffImg discovered $40 \%$ of the 100,000 fake SNe Ia light curves that were inserted on the DES-SN images, and the SMP pipeline was run on a representative subset of 10,000 light curves. Analysis requirements and SALT2 light-curve fitting resulted in a sample of 6586 fake SNe Ia that are fit with BBC and CosmoMC.

For the BBC fit we create a bias correction sample from SNANA simulations (SIM2). The underlying SN Ia light-curve model is identical to that used in SIM1-for example, color and stretch population, and no intrinsic scatter. In the first season (Y1), there was a SIM1 generation bug forcing the same galactic extinction $(E(B-V=0.043)$ at all $C C D$ image locations, and this same bug was intentionally preserved in SIM2 for Y1. In contrast to the real data, $E_{\text {spec }}=1$ for both SIM1 and SIM2.

Finally, the SIM2 redshift distribution was tuned in each of the ten SN fields to match SIM1 after cuts. This field-dependent redshift tuning was needed because of the subtle way that SIM1 had selected real host galaxies from the science verification (SV) catalog. Although a single host-galaxy $z$ dependence was specified, the non-uniform depth of the SV galaxy catalog resulted in a different redshift distribution in each field. To illustrate this feature, consider an extreme example with just two fields (e.g., E1, E2). Next, suppose that the galaxy catalog for E1 only includes redshifts $z<0.5$ while for E2 we have $z>0.5$. A simulation generating a flat galaxy redshift distribution over $0<z<1$ results in non-overlapping (i.e., different) redshift distributions in E1 and E2. A comparison between the resulting bias correction simulation and the fakes is shown in Figure 18.

\section{ORCID iDs}

D. Brout (1) https://orcid.org/0000-0001-5201-8374

R. Kessler (i) https://orcid.org/0000-0003-3221-0419

C. B. D'Andrea (ib https://orcid.org/0000-0002-8198-0332

S. R. Hinton (1) https://orcid.org/0000-0003-2071-9349

C. Lidman (1) https://orcid.org/0000-0003-1731-0497

K. Glazebrook (i) https://orcid.org/0000-0002-3254-9044

G. F. Lewis (1) https://orcid.org/0000-0003-3081-9319

E. Morganson (1) https://orcid.org/0000-0001-7180-109X

D. Muthukrishna (i) https://orcid.org/0000-0002-5788-9280

P. Nugent (i) https://orcid.org/0000-0002-3389-0586

A. Palmese (i) https://orcid.org/0000-0002-6011-0530

R. Sharp (1) https://orcid.org/0000-0003-4877-7866

J. Annis (ㄷ) https://orcid.org/0000-0002-0609-3987

F. J. Castander (1) https://orcid.org/0000-0001-7316-4573

A. Drlica-Wagner (i) https://orcid.org/0000-0001-8251-933X

R. A. Gruendl (1) https://orcid.org/0000-0002-4588-6517
D. L. Hollowood (1) https://orcid.org/0000-0002-9369-4157

P. Martini (i) https://orcid.org/0000-0002-4279-4182

R. Miquel (i) https://orcid.org/0000-0002-6610-4836

M. Soares-Santos $\mathbb{1}$ https://orcid.org/0000-0001-6082-8529

G. Tarle (i) https://orcid.org/0000-0003-1704-0781

A. R. Walker (i) https://orcid.org/0000-0002-7123-8943

\section{References}

Abbott, T. M. C., Abdalla, F. B., Allam, S., et al. 2018, ApJS, 239, 18 Arnouts, S., \& Ilbert, O. 2011, LePHARE: Photometric Analysis for Redshift Estimate, Astrophysics Source Code Library, ascl:1108.009

Astier, P., El Hage, P., Guy, J., et al. 2013, A\&A, 557, A55

Astier, P., Guy, J., Regnault, N., et al. 2006, A\&A, 447, 31

Bernstein, G. M., Abbott, T. M. C., Desai, S., et al. 2017, PASP, 129, 114502

Bernstein, J. P., Kessler, R., Kuhlmann, S., et al. 2012, ApJ, 753, 152

Betoule, M., Kessler, R., Guy, J., et al. 2014, A\&A, 568, A22

Blondin, S., \& Tonry, J. L. 2007, ApJ, 666, 1024

Bohlin, R. C., Gordon, K. D., \& Tremblay, P.-E. 2014, PASP, 126, 711

Bonnett, C., Troxel, M. A., Hartley, W., et al. 2016, PhRvD, 94, 042005

Brout, D. 2018, AAS Meeting, 231, 219.04

Brout, D., Sako, M., Scolnic, D., et al. 2018, arXiv:1811.02378

Bruzual, G., \& Charlot, S. 2003, MNRAS, 344, 1000

Burke, D. L., Rykoff, E. S., Allam, S., et al. 2018, AJ, 155, 41

Calcino, J., \& Davis, T. 2017, JCAP, 1, 038

Carrick, J., Turnbull, S. J., Lavaux, G., \& Hudson, M. J. 2015, MNRAS, 450,317

Childress, M., Aldering, G., Antilogus, P., et al. 2013, ApJ, 770, 107

Childress, M. J., Lidman, C., Davis, T. M., et al. 2017, MNRAS, 472, 273

Childress, M. J., Wolf, C., \& Zahid, H. J. 2014, MNRAS, 445, 1898

Chotard, N., Gangler, E., Aldering, G., et al. 2011, A\&A, 529, L4

Conley, A., Guy, J., Sullivan, M., et al. 2011, ApJS, 192, 1

Contreras, C., Hamuy, M., Phillips, M. M., et al. 2010, AJ, 139, 519

Dai, M., \& Wang, Y. 2016, MNRAS, 459, 1819

D’Andrea, C., Smith, M., Sullivan, M., et al. 2018, arXiv:1811.09565

D'Andrea, C. B., Gupta, R. R., Sako, M., et al. 2011, ApJ, 743, 172

DES Collaboration, A., Abbott, T. M. C., Allam, S., et al. 2018, arXiv:1811.02374

Diehl, H. T., Abbott, T. M. C., Annis, J., et al. 2014, Proc. SPIE, 9149, 91490V

Diehl, H. T., Angstadt, R., Campa, J., et al. 2008, Proc. SPIE, 7021, 702107

Doi, M., Tanaka, M., Fukugita, M., et al. 2010, AJ, 139, 1628

Fioc, M., \& Rocca-Volmerange, B. 1997, A\&A, 326, 950

Fitzpatrick, E. L. 1999, PASP, 111, 63

Flaugher, B., Diehl, H. T., Honscheid, K., et al. 2015, AJ, 150, 150

Foley, R. J., Scolnic, D., Rest, A., et al. 2018, MNRAS, 475, 193

Frieman, J. A., Bassett, B., Becker, A., et al. 2008, AJ, 135, 338

Gallagher, J. S., Garnavich, P. M., Caldwell, N., et al. 2008, ApJ, 685, 752

Goldstein, D. A., D’Andrea, C. B., Fischer, J. A., et al. 2015, AJ, 150, 82

Gupta, R. R., D’Andrea, C. B., Sako, M., et al. 2011, ApJ, 740, 92

Gupta, R. R., Kuhlmann, S., Kovacs, E., et al. 2016, AJ, 152, 154

Guy, J., Astier, P., Baumont, S., et al. 2007, A\&A, 466, 11

Guy, J., Sullivan, M., Conley, A., et al. 2010, A\&A, 523, A7

Hicken, M., Challis, P., Kirshner, R. P., et al. 2012, ApJS, 200, 12

Hicken, M., Wood-Vasey, W. M., Blondin, S., et al. 2009a, ApJ, 700, 1097

Hinton, S. R., Davis, T. M., Kim, A. G., et al. 2018, arXiv:1811.02381

Hinton, S. R., Davis, T. M., Lidman, C., Glazebrook, K., \& Lewis, G. F. 2016, A\&C, 15, 61

Holland, S. E., Groom, D. E., Palaio, N. P., Stover, R. J., \& Wei, M. 2003, ITED, 50, 225

Holtzman, J. A., Marriner, J., Kessler, R., et al. 2008, AJ, 136, 2306

Hounsell, R., Scolnic, D., Foley, R. J., et al. 2018, ApJ, 867, 23

Howell, D. A., Sullivan, M., Perrett, K., et al. 2005, ApJ, 634, 1190

Ivezić, Z., Kahn, S. M., Tyson, J. A., et al. 2008, arXiv:0805.2366

James, F., \& Roos, M. 1975, CoPhC, 10, 343

Jha, S., Kirshner, R. P., Challis, P., et al. 2006, AJ, 131, 527

Jones, D. O., Scolnic, D. M., Riess, A. G., et al. 2018, ApJ, 857, 51

Kelly, P. L., Hicken, M., Burke, D. L., Mandel, K. S., \& Kirshner, R. P. 2010, ApJ, 715, 743

Kessler, R., Becker, A. C., Cinabro, D., et al. 2009a, ApJS, 185, 32

Kessler, R., Bernstein, J. P., Cinabro, D., et al. 2009b, PASP, 121, 1028

Kessler, R., Brout, D., D’Andrea, C. B., et al. 2019, MNRAS, 485, 1171

Kessler, R., Marriner, J., Childress, M., et al. 2015, AJ, 150, 172

Kessler, R., \& Scolnic, D. 2017, ApJ, 836, 56 
Lampeitl, H., Smith, M., Nichol, R. C., et al. 2010, ApJ, 722, 566

Lasker, J., Kessler, R., Scolnic, D., et al. 2019, MNRAS, tmp, 607

Le Borgne, D., \& Rocca-Volmerange, B. 2002, A\&A, 386, 446

Lewis, A., \& Bridle, S. 2002, PhRvD, 66, 103511

Li, T., DePoy, D. L., Marshall, J. L., et al. 2014, Proc. SPIE, 9147, $91476 Z$

LSST Science Collaboration, Abell, P. A., Allison, J., et al. 2009, arXiv:0912.0201

March, M. C., Trotta, R., Berkes, P., Starkman, G. D., \& Vaudrevange, P. M. 2011, MNRAS, 418, 2308

Marshall, J. L., Rheault, J.-P., DePoy, D. L., et al. 2013, arXiv:1302.5720

Morganson, E., Gruendl, R. A., Menanteau, F., et al. 2018, PASP, 130, 074501

Narayan, G., Rest, A., Tucker, B. E., et al. 2016, ApJS, 224, 3

Oke, J. B., \& Gunn, J. E. 1983, ApJ, 266, 713

Pan, Y.-C., Sullivan, M., Maguire, K., et al. 2014, MNRAS, 438, 1391

Perlmutter, S., Aldering, G., Goldhaber, G., et al. 1999, ApJ, 517, 565

Perrett, K., Balam, D., Sullivan, M., et al. 2010, AJ, 140, 518

Planck Collaboration 2016, A\&A, 594, A13

Rest, A., Scolnic, D., Foley, R. J., et al. 2014, ApJ, 795, 44

Riess, A. G., Filippenko, A. V., Challis, P., et al. 1998, AJ, 116, 1009

Riess, A. G., Kirshner, R. P., Schmidt, B. P., et al. 1999, AJ, 117, 707

Rigault, M., Aldering, G., Kowalski, M., et al. 2015, ApJ, 802, 20

Rigault, M., Brinnel, V., Aldering, G., et al. 2018, arXiv:1806.03849

Rigault, M., Copin, Y., Aldering, G., et al. 2013, A\&A, 560, A66

Rubin, D., Aldering, G., Barbary, K., et al. 2015, ApJ, 813, 137
Sako, M., Bassett, B., Becker, A. C., et al. 2018, PASP, 130, 064002

Schlafly, E. F., \& Finkbeiner, D. P. 2011, ApJ, 737, 103

Schlafly, E. F., Finkbeiner, D. P., Schlegel, D. J., et al. 2010, ApJ, 725, 1175

Schlegel, D. J., Finkbeiner, D. P., \& Davis, M. 1998, ApJ, 500, 525

Scolnic, D., Casertano, S., Riess, A., et al. 2015, ApJ, 815, 117

Scolnic, D., \& Kessler, R. 2016, ApJL, 822, L35

Scolnic, D., Rest, A., Riess, A., et al. 2014, ApJ, 795, 45

Scolnic, D. M., Jones, D. O., Rest, A., et al. 2018, ApJ, 859, 101

Shariff, H., Jiao, X., Trotta, R., \& van Dyk, D. A. 2016, ApJ, 827, 1

Skrutskie, M. F., Cutri, R. M., Stiening, R., et al. 2006, AJ, 131, 1163

Smith, M., Nichol, R. C., Dilday, B., et al. 2012, ApJ, 755, 61

Sullivan, M., Conley, A., Howell, D. A., et al. 2010, MNRAS, 406, 782

Sullivan, M., Guy, J., Conley, A., et al. 2011, ApJ, 737, 102

Sullivan, M., Le Borgne, D., Pritchet, C. J., et al. 2006, ApJ, 648, 868

Tanabashi, M., Hagiwara, K., Hikasa, K., et al. 2018, PhRvD, 98, 030001

Tonry, J. L., Stubbs, C. W., Lykke, K. R., et al. 2012, ApJ, 750, 99

Tripp, R. 1998, A\&A, 331, 815

Wojtak, R., Davis, T. M., \& Wiis, J. 2015, JCAP, 7, 025

Wolf, R. C., D’Andrea, C. B., Gupta, R. R., et al. 2016, ApJ, 821, 115

Wood-Vasey, W. M., Miknaitis, G., Stubbs, C. W., et al. 2007, ApJ, 666 , 694

Yuan, F., Lidman, C., Davis, T. M., et al. 2015, MNRAS, 452, 3047

Zhang, B. R., Childress, M. J., Davis, T. M., et al. 2017, MNRAS, 471, 2254 